\title{
THE COLLISIONAL EVOLUTION OF ORBITAL DEBRIS IN GEOPOTENTIAL WELLS AND DISPOSAL ORBITS
}

\author{
A Thesis \\ presented to \\ the Faculty of California Polytechnic State University, \\ San Luis Obispo
}

\author{
In Partial Fulfillment \\ of the Requirements for the Degree \\ Master of Science in Aerospace Engineering
}

by

Benjamin Polzine

March 2017 
(C) 2017

Benjamin Polzine

ALL RIGHTS RESERVED 


\section{COMMITTEE MEMBERSHIP}

TITLE:

AUTHOR:

DATE SUBMITTED:

COMMITTEE CHAIR
The Collisional Evolution of Orbital Debris in Geopotential Wells and Disposal Orbits

Benjamin Polzine

March 2017

COMMITTEe MEMBER: Professor Gary Hughes, Ph.D.

Department of Statistics

COMMITTEE MEMBER: Paula Krisko, Ph.D NASA Johnson Space Center Orbital Debris Modeling Specialist

COMMITTEE MEMBER: Professor Eric Mehiel, Ph.D. Department of Aerospace Engineering 


\begin{abstract}
The Collisional Evolution of Orbital Debris in Geopotential Wells and Disposal Orbits

Benjamin Polzine
\end{abstract}

This thesis investigates the orbital debris evolution in the geosynchronous disposal orbit regime and within geosynchronous orbits effected by the geopotential wells. A propagator is developed for the accurate simulation of GEO specific orbits and the required perturbations are determined and described. Collisions are then simulated in the selected regimes using a low velocity breakup model derived from the NASA EVOLVE breakup model. The simulations described in this thesis consider a set of perturbations including the geopotential, solar and lunar gravity, and solar radiation pressure forces. This thesis is based on a prior paper and additionally seeks to address an issue in simulating East-West trapped objects. The results show that this propagator successfully simulates the presence of all wells and the East-West entrapment, and the required perturbations are outlined. Five collision test cases were simulated, one for each type of entrapment and an additional for the disposal orbit. 


\section{ACKNOWLEDGMENTS}

Thanks to:

- Dr. Kira Abercromby, for helping to lay a path through the arduous world of aerospace.

- Dr. Eric Mehiel, Dr. Gary Hughes, and Dr. Paula Krisko for providing their time, insight, and patience I needed to work through this paper.

- Miriam Krage, for keeping me sane.

- Andrew Guenther, for sharing a LaTeX template for the Cal Poly thesis format.

- All of the family, loved ones, and faculty that not only supported me through my years in college but continue to support me on the long road ahead.

- Ben 


\section{TABLE OF CONTENTS}

$\begin{array}{lll}\text { Page } & \text { Paj }\end{array}$

LIST OF TABLES . . . . . . . . . . . . . . . . . v viii

LIST OF FIGURES ..................... . . ix CHAPTER

1 Introduction . . . . . . . . . . . . . . . . 1

1.1 Orbital Debris . . . . . . . . . . . . . . . . 2

1.1.1 Orbital Debris Collisions . . . . . . . . . . . . 3

1.2 GEO Region . . . . . . . . . . . . . . . . . . 4

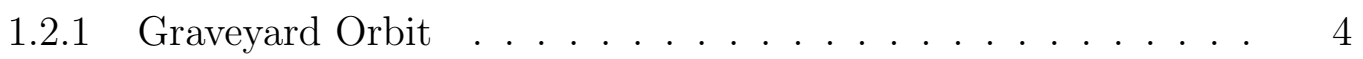

1.2.2 Geopotential Wells ................. 6

1.3 Two-Line Element Set . . . . . . . . . . . . . . . 8

1.4 Prior Work in Debris and Debris Modelling . . . . . . . . . . . . 10

1.5 Thesis Proposal . . . . . . . . . . . . . . . . . . . . 11

2 Orbital Propagator . . . . . . . . . . . . . . . . 12

2.1 Methods . . . . . . . . . . . . . . . . . 12

2.1.1 Cowell's Method . . . . . . . . . . . . . 13

2.1.2 Encke's Method . . . . . . . . . . . . . . . . . . 14

2.1.3 Variation of Parameters Method . . . . . . . . . . . . 15

2.1.3.1 True and Modified Classical Element Set . . . . . . 15

2.1.3.2 Equinoctial Element Set . . . . . . . . 16

2.1.3.3 Modified Equinoctial Element Set . . . . . . . 16

2.1.3.4 Chosen Approach . . . . . . . . . . . . 17

2.2 Perturbation Analysis . . . . . . . . . . . . . . . 18

2.2.1 Earth Oblateness . . . . . . . . . . . . . . . 20

2.2.1.1 $J_{2}$ Perturbation . . . . . . . . . . . . . . . . 21 


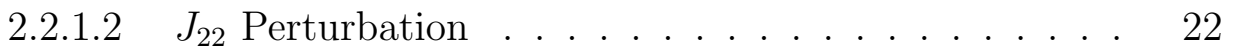

2.2.1.3 Additional Geopotential Perturbations . . . . . . . . 24

$2.2 .2 \quad$ Solar Body Effects . . . . . . . . . . . . . . . . 25

2.2 .3 Lunar Body Effects . . . . . . . . . . . . . . . . . . . . 27

2.2.4 Solar Radiation Pressure . . . . . . . . . . . . . . . . . . . . . 29

2.2.5 Excluded Perturbations . . . . . . . . . . . . . . . . 31

2.3 MATLAB Environmental Variables . . . . . . . . . . . . . . . 32

2.4 Using the Propagator . . . . . . . . . . . . . . . . . . . 33

3 Collision Simulation . . . . . . . . . . . . . . . . . 35

3.1 Previous Work . . . . . . . . . . . . . . . . . . 35

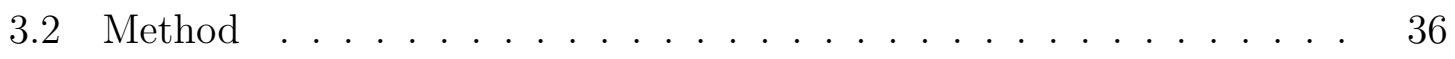

3.3 Using the Collision Code . . . . . . . . . . . . . . . . . . . . 39

4 Results . . . . . . . . . . . . . . . . . 40

4.1 Propagator Validation . . . . . . . . . . . . . . 40

4.1.1 Comparison Plots - STK and Custom Propagators . . . . . . . 42

4.1.2 Comparison Plots - No-SRP Equinoctial Propagator . . . . . . 52

4.1.3 Comparison Plots - TLEs, STK, and Cowell 3x3 . . . . . . 55

4.2 Collision Simulation Validation _. . . . . . . . . . . . 60

4.3 Combined Simulation Results . . . . . . . . . . . . . . . . 62

5 Conclusion . . . . . . . . . . . . . . . . . . . . 69

5.1 Future Work . . . . . . . . . . . . . . . . . . . . . . . 71

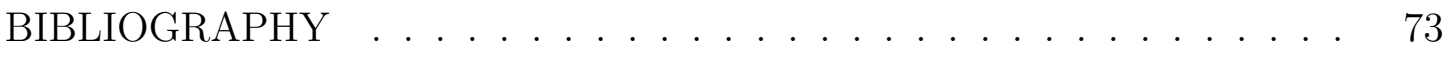
APPENDICES

A. No-SRP Equinoctial Variation . . . . . . . . . . . . . . 77

B. Comparing Cowell Propagators . . . . . . . . . . . . . . . . 81 


\section{LIST OF TABLES}

Table

1.1 First Line of a TLE . . . . . . . . . . . . . . . 9

1.2 Second Line of a TLE . . . . . . . . . . . . . . . . . . . . . 9

2.1 Modified Classical Element Set . . . . . . . . . . . 16

2.2 Equinoctial Element Set . . . . . . . . . . . . . . . . . 16

2.3 Modified Equinoctial Element Set . . . . . . . . . . . . . . 17

2.4 Perturbation Order of Influence . . . . . . . . . . . . . . . . 19

2.5 Environmental Variables . . . . . . . . . . . . . . 33

4.1 Set of Satellites Used For Testing . . . . . . . . . . . . 41

4.2 Graveyard Object State . . . . . . . . . . . . . 67 


\section{LIST OF FIGURES}

Figure $\quad$ Page

1.1 Minimum Recommended Perigee of the Graveyard Orbit . . . . . . 6

1.2 Stable (Green) and Unstable (Red) Geopotential Wells . . . . . . . 7

1.3 Criterion for capture by a geopotential well $[23] \ldots \ldots$

2.1 Osculating and Reference Orbits of Encke's Method . . . . . . . . . 14

2.2 The three types of spherical harmonics . . . . . . . . . . . . . . 21

2.3 Vectors for Solar Body Effects . . . . . . . . . . . . . . . 25

2.4 Vectors for Lunar Body Effects . . . . . . . . . . . . . . 28

2.5 Shadow Function Angles $[6] \ldots . . \ldots 31$

2.6 A layout of all elements of the code . . . . . . . . . . . . 34

4.1 Short Term Versus Plots for Satellite 03432 . . . . . . . . . . . . . 44

4.2 Long Term Versus Plots for Satellite 03432 . . . . . . . . . . . . . 44

4.3 Short Term Versus Plots for Satellite 04068 . . . . . . . . . . 45

$4.4 \quad$ Long Term Versus Plots for Satellite 04068 . . . . . . . . . . . . 46

4.5 Short Term Versus Plots for Satellite 04250 . . . . . . . . . . . 47

4.6 Long Term Versus Plots for Satellite 04250 . . . . . . . . . . . 47

4.7 Short Term Versus Plots for Satellite 05588 . . . . . . . . . . . . . 48

4.8 Long Term Versus Plots for Satellite 05588 . . . . . . . . . . . . 49

4.9 Short Term Versus Plots for Satellite 29640 . . . . . . . . . . . . . 50

4.10 Long Term Versus Plots for Satellite 29640 . . . . . . . . . . . . 51

4.11 Long Term Versus Plots for Satellite 05588 with 3x3 Geopotential for Cowell . . . . . . . . . . . . . . . . . . . . 52

4.12 SRP Variation in Curtis, Example $12.7 \ldots \ldots . \ldots . . \ldots 54$

4.13 Long Term Plots for Satellite $03432 \ldots \ldots$. . . . . . . . . 56 
4.14 Longitude trend underlying behavior . . . . . . . . . . . 56

4.15 Long Term Plots for Satellite 04068 . . . . . . . . . . . . . 57

4.16 Long Term Plots for Satellite $04250 \ldots \ldots \ldots$

4.17 Long Term Plots for Satellite 05588 . . . . . . . . . . . . . . 59

4.18 Long Term Plots for Satellite $29640 \ldots \ldots$. . . . . . . . . . 60

4.19 The $\Delta v$ distribution of fragments for two objects of $1000 \mathrm{~kg}$ and 400 $\mathrm{kg}$ during a $300 \mathrm{~m} / \mathrm{s}$ collision $\ldots \ldots \ldots . \ldots . \ldots 61$

4.20 Conceptual outline of collision simulator usage . . . . . . . . . 62

4.21 Longitudinal Fragmentation Behavior - Satellite 04068 . . . . . . . 63

4.22 Longitudinal Fragmentation Behavior - Satellite 04250 . . . . . . 64

4.23 Longitudinal Fragmentation Behavior - Satellite 05588 . . . . . . 65

4.24 Longitudinal Fragmentation Behavior - Satellite 29640 . . . . . . 66

4.25 Longitudinal Fragmentation Behavior - Graveyard Satellite . . . . . 68

A.1 Long Term No-SRP Plots for Satellite $03432 \ldots \ldots \ldots \ldots$. . . . 77

A.2 Long Term No-SRP Plots for Satellite $04068 \ldots \ldots \ldots \ldots$

A.3 Long Term No-SRP Plots for Satellite $04250 \ldots \ldots$. . . . . . 79

A.4 Long Term No-SRP Plots for Satellite $05588 \ldots \ldots$. . . . . . . 80

A.5 Long Term No-SRP Plots for Satellite $29640 \ldots \ldots$. . . . . . . 80

B.1 Cowell Comparison Plots for Satellite $03432 \ldots \ldots \ldots$

B.2 Cowell Comparison Plot for Satellite $04068 \ldots \ldots \ldots \ldots$. . . . 82

B.3 Cowell Comparison Plot for Satellite $04250 \ldots \ldots \ldots$. . . . . . 82

B.4 Cowell Comparison Plot for Satellite $05588 \ldots \ldots \ldots$

B.5 Cowell Comparison Plot for Satellite $29640 \ldots \ldots \ldots \ldots$. . . . . 83 


\section{Chapter 1}

\section{Introduction}

When considering the Earth's space environment for a potential spacecraft mission, it is important to consider orbital debris and its potential impact on the health and well-being of the spacecraft involved. As a growing number of objects are launched into space for operations, the threat of potential collisions between objects in orbit has become a very real risk. In addition to the active satellites fulfilling their mission duties there is an even greater body of orbiting objects including decommissioned satellites, rocket bodies, payload fairings, paint flecks, and all manner of other uncontrolled ejecta.

A number of organizations around the world seek to track and determine the orbital characteristics of this debris so they may protect active spacecraft and understand any adverse risks posed to the health of the space environment. Some of these organizations include NASA and its Orbital Debris Program Office at Johnson Space Center, the U.S. Strategic Command, and the European Space Agency. When impacting another object, debris can result in hyper-velocity collisions with an average velocity of $10 \mathrm{~km} / \mathrm{s}$ in lower altitude orbits. In these scenarios, even small objects like like paint flecks or bolts can pose a serious danger to spacecraft. Travelling at these speeds, even an object as small as $1 \mathrm{~cm}$ in size could break through the shielding of the International Space Station [25], [34].

When considering the lifetime of these debris objects, the forces and attributes of each element of debris has to be taken into account. In low-altitude regimes, those below $800 \mathrm{~km}$ in altitude, debris will often be pulled into the atmosphere by perturbative forces and reenter within a few decades. Debris located in orbits higher 
than this will remain for even longer - an object trapped for centuries in the orbit it was deposited in is entirely possible. As additional spacecraft are launched into these high-orbit regions, the object density at these regimes continue to increase and with it the chance of a collision. Further troubling is that the standard for obsolete satellites in regimes like the geosynchronous Earth orbit (GEO) is to move the satellite into a higher orbit deemed the "graveyard orbit." Whereas a Low Earth Orbit (LEO) spacecraft can reenter the atmosphere, GEO satellites raise their altitude due to fuel constraints. The end result is a belt of uncontrolled debris packed closely behind the active GEO orbit, posing an ever growing probability of collision. Furthermore, the standards for placing objects into the graveyard orbit are not always maintained, resulting in orbits that may draw close enough to the GEO regime to pose a threat to active satellites in the area. Exacerbating the problem further are geopotential "sinks", areas in the graveyard orbit that result in longitudinally trapped debris. These sinks affect the orbital behavior of the graveyard orbit, resulting in a regime of relatively unknown and complex behavior that poses concern for the well-being of the high traffic GEO region of space.

\section{$1.1 \quad$ Orbital Debris}

Whether as a result of operations, accident, or intention, the continued generation of space debris is very much a constant danger in near-earth space operations. It was not always like this; the space around Earth was entirely free of man-made objects until Sputnik 1 was launched in 1957. With a relatively low orbit, Sputnik 1 was dragged into re-entry by Earth's atmosphere in only a month. The true accumulation of debris did not begin until 1957 with the launch of the Vanguard satellites. Vanguard 1 in 1957, along with 2 other Vanguard satellites launched in 1959, was launched into a medium earth orbit (MEO) and decommissioned after 6 years of activity [12]. When the active lifespan of these satellites ended, Vanguard 1 and its successors were left in their orbits. With no significant forces to pull these satellites down, they are expected to remain in orbit for another 200 years, marking the first long-term debris to be placed in Earth's sphere of influence. With catalogs of space objects being maintained by numerous governments and organizations, an understanding for just 
how much debris there is can be hinted at. According to the Orbital Debris Quarterly News article published by NASA, of a total of nearly 18,000 objects tracked by the U.S. Space Surveillance Network, roughly 14,000 of those are fragmentation debris, mission-related debris, or expended rocket bodies [24]. With the remaining 4,000 objects being some form of payload, debris vastly outnumbers functional satellites and collision probability trends have reflected that growing threat. In 1981 the probability of collision between any two objects was rated at $6 \%$ per year [19], but by 2013 the likelihood of collision for a spacecraft and debris larger than $1 \mathrm{~cm}$ in size was $50-67 \%$ per year [33]. With such growth in collision probability and the continued interest in space activity, a great demand has been raised for an accurate understanding of debris behavior and how it might effect the future of space operations.

\subsubsection{Orbital Debris Collisions}

There are two types of collisions in space: the intentional and the accidental. With collisional velocities up to $10 \mathrm{~km} / \mathrm{s}$ or more in LEO [26] and $800 \mathrm{~m} / \mathrm{s}$ in GEO [13], collisions even with objects as small and insignificant as paint flecks are dangerous. One of the best examples of this is the damage caused by a $0.76 \mathrm{~mm}$ diameter, 0.3 mm thick paint chip after it impacted the Space Shuttle Discovery on STS-92 [15]. A $10 \mathrm{~mm}$ diameter and $2 \mathrm{~mm}$ deep crater was left in the left hand 2 window and a replacement had to be made because of the potential for future growth in the flaw. In 2009, the accidental collision of an Iridium satellite and Russian comm satellite Cosmos 2251 resulted in over 1,600 unique pieces of debris that not only endangered nearby satellites but pose a future threat to lower altitude vehicles such as the ISS as the cloud of debris reenters over the next 30 years [36].

Looking at intentional debris production, the beginnings lie with the US and USSR between the 1968 and 1985. Both groups experimented with anti-satellite weapons that either destroyed satellites with their own debris or by directly impacting a target with a new satellite. The result of these tests left hundreds of debris objects in Earthbound space. Since then, only five known anti-satellite launches have been conducted: two by China in 2007 and 2013, one by the US in 2008, and two by Russia in 2015 and 2016. The most noteworthy of these is the 2007 Chinese intercept and destruction 
of Fengyun-1C. At an altitude of $863 \mathrm{~km}$, the intentional destruction of this satellite resulted in over 3,000 distinct pieces of debris and raised the total number of debris objects from roughly 10,000 to 13,000 . Within only three years, the debris from this collision had managed to scatter across an altitude range of $175 \mathrm{~km}$ to $3,600 \mathrm{~km}$ and has come to impact the safety of some of the most active orbits around Earth [37].

\subsection{GEO Region}

For the purpose of this thesis, the analysis of the orbital debris environment will be restricted to the GEO region and its neighboring, higher-altitude Graveyard Orbit. The geosynchronous earth orbit is a highly lucrative, high-altitude orbit with an altitude of approximately $35,786 \mathrm{~km}$. Satellites in this orbit complete one period of their orbit at the same rate as the Earth rotates about its axis. This results in what looks to be a figure-eight pattern for the satellites ground track, centered around a longitude and moving up and down in latitude. A special case of this orbit is the geostationary equatorial orbit, a circular GEO in the plane of the Earth's equator. Satellites in this orbit hold a fixed latitude and longitude relative to the Earth's surface, at least when neglecting perturbatory effects or active orbit maintenance is undertaken. This is an especially valuable orbit as it allows for an object to remain in the same position in the sky relative to any location on Earth's surface. One example application of this orbit is satellite television, which relies on users to point a receiving dish to one spot in the sky and receive television broadcasts without having to reorient the dish every day.

\subsubsection{Graveyard Orbit}

When GEO spacecraft have reached their end-of-life, three options are presented to operators. The soon-to-be defunct spacecraft can:

a) be left in its current location. For a geosynchronous orbit, this means occupying one of a limited number of longitudinal locations before, due to perturbatory forces, drifting into other longitude bins and potentially disrupting or colliding with other spacecraft. Due to the potential risks and costs involved with the 
consequences of this action this is disapproved of, however a satellite suffering from malfunction may inadvertently end up in this situation.

b) alter its trajectory to burn-up in the atmosphere. This is a costly maneuver that is on the order of $\mathrm{km} / \mathrm{s}$ in delta-v for a standard GEO satellite. As the delta- $v$ is no insignificant figure, the spacecraft will require a sizable reserve stock of fuel to deorbit properly. Additionally, reentry analysis must be done to confirm the risk of human fatality does not exceed 1 in 10,000. This method is too fuel intensive to be considered for a typical GEO spacecraft.

c) conduct a series of small burns to either raise or lower the orbit into a designated graveyard orbit. The easiest maneuver for a GEO would be to raise the orbit as this is a lower energy maneuver and require the least fuel. Compared to deorbiting, these burns should require only tens of meters per second of delta-v.

When comparing these options, the most common and practical choice is to burn into a graveyard orbit. The recommended graveyard orbit specifications, as provided by the Inter-Agency Space Debris Coordination Committee (IADC), is defined by a minimum perigee altitude change of

$$
\Delta H=235 k m+\left(1000 C_{R} \frac{A}{m}\right) \quad[k m]
$$

where $C_{R}$ is the solar radiation pressure coefficient, and $\frac{A}{m}$ is the area-to-mass ratio in $m^{2} / \mathrm{kg}$ for the spacecraft [5]. This definition provides a region of about $200 \mathrm{~km}$ of spacing between the GEO orbit and the graveyard orbit. A visualization of this equation is provided in Figure 1.1.

Despite this spacing, not all spacecraft operators follow these guidelines or have the means to do so. This results in irregular orbits that may overlap both regimes and pose an unexpected risk to the GEO orbit. As debris objects, many of these lack any capability to be controlled in the event of a possible collision or breakup. 


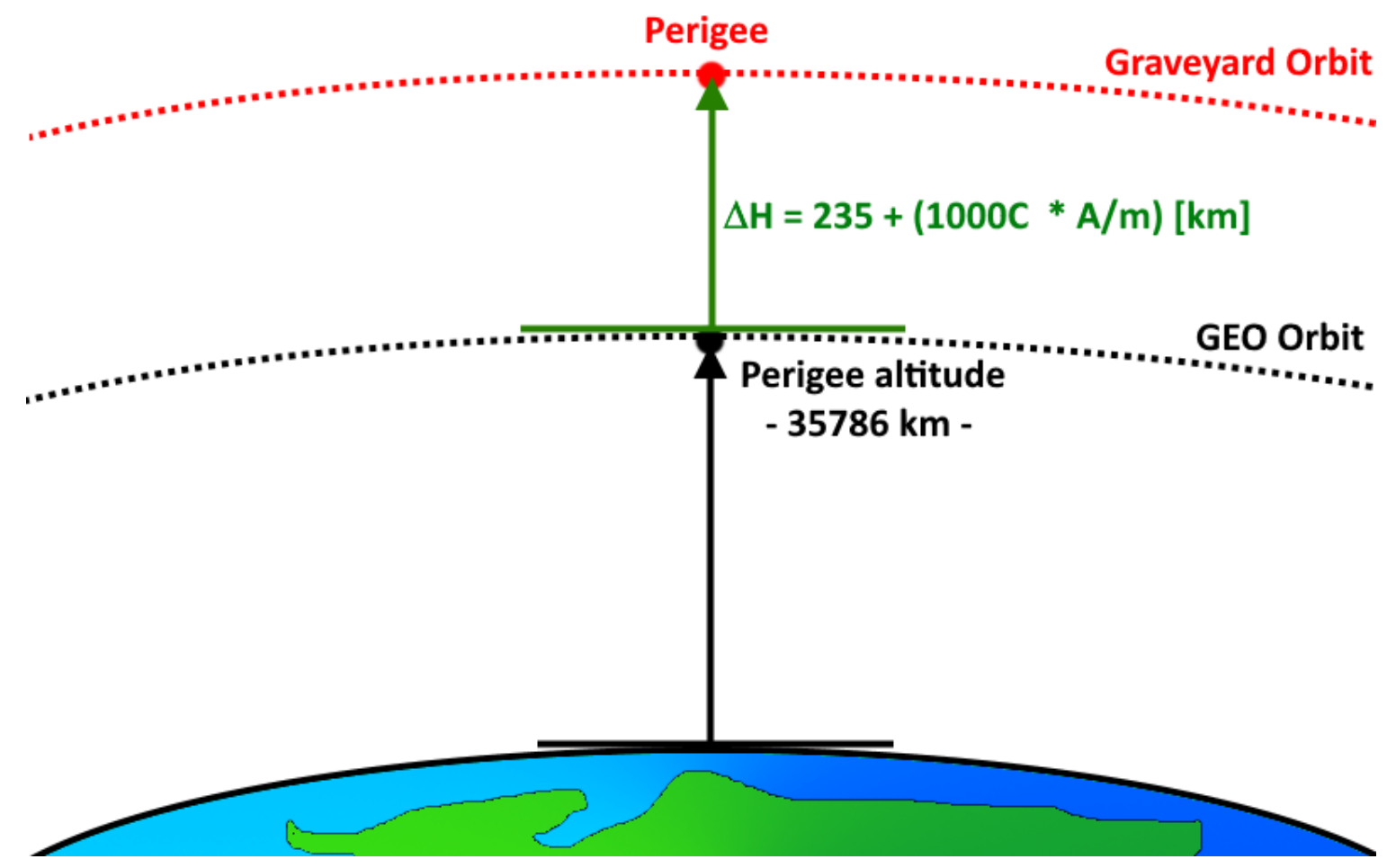

Figure 1.1: Minimum Recommended Perigee of the Graveyard Orbit

\subsubsection{Geopotential Wells}

As a result of the aspherocity of Earth and its non-symmetric mass distribution, a group of geopotential "wells" and "hills" exist in the space around Earth. These areas represent locations where a satellites acceleration is zero - they are equilibrium points within the system where wells act as the stable locations and hills are the unstable locations. For a geostationary satellite, there are two of each of these as depicted in Figure 1.2. The unstable points are located at $11.5^{\circ} \mathrm{W}$ and $161.9^{\circ} \mathrm{E}$. As unstable points, slight perturbations are capable of moving a satellite away from this hills meaning that without some form of propulsion to maintain a satellites orbit, the satellite will slowly drift away from this point. The stable points, located at $75.1^{\circ} \mathrm{E}$ and $105.3^{\circ} \mathrm{W}$ longitude, are capable of pulling satellites near the node into an oscillating orbit about the well's center. The nature of a geopotential well can change the behavior of debris by redirecting the debris during its orbit. Whereas an operational satellite can utilize east-west station keeping to maintain its orbit, debris is subjected to these forces and unexpected behavior can result. 


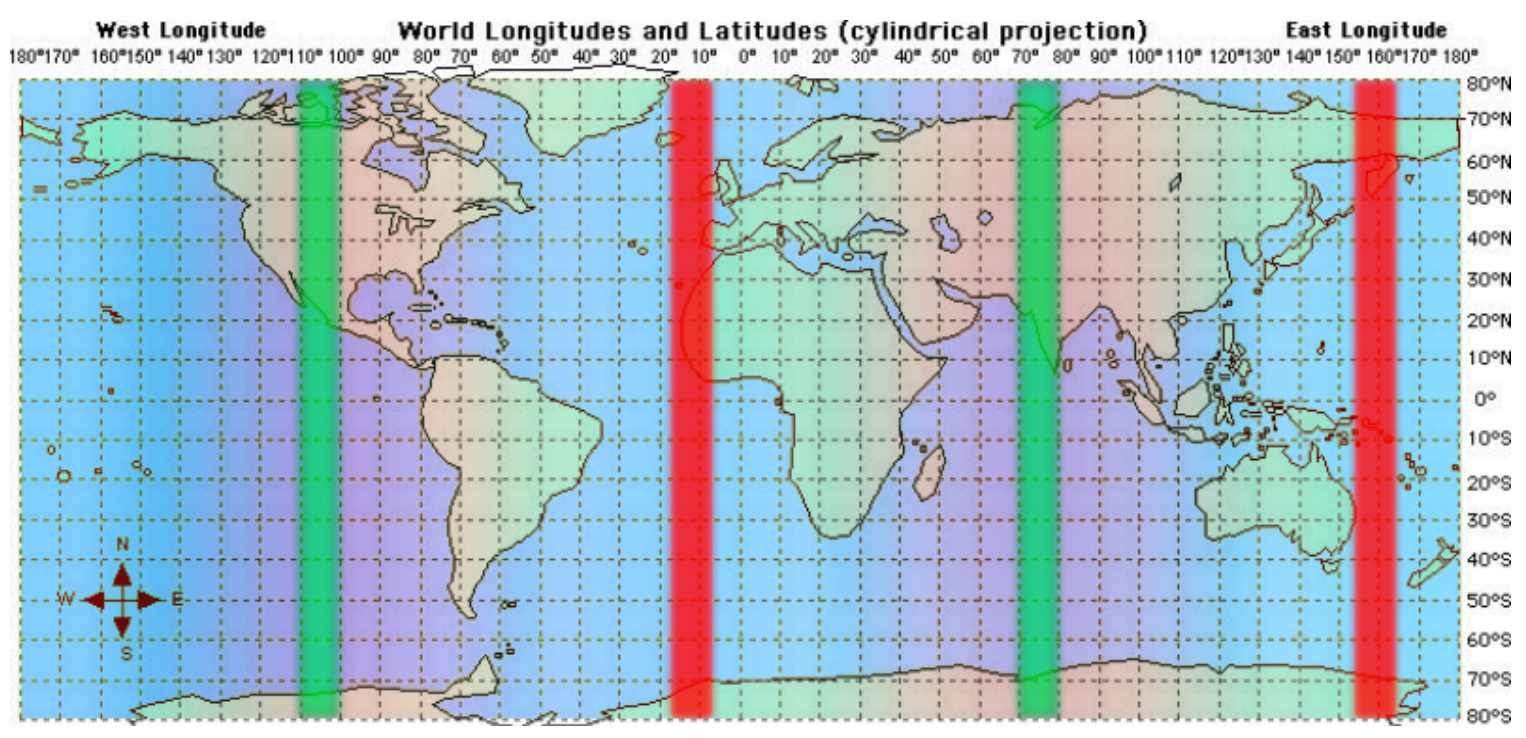

Figure 1.2: Stable (Green) and Unstable (Red) Geopotential Wells

Four types of debris exist when considering the effect of geopotential wells: drifters, trapped debris in the East well, trapped debris in the West well, and dual-trapped debris. Drifters are objects that are not trapped by either well, meaning they are not trapped to a range of longitudes. These objects will, over time, drift around the Earth and do not maintain a fixed longitude. West trapped debris is bound by roughly $0^{\circ}$ $\mathrm{W}$ and $180^{\circ} \mathrm{W}$, whereas an East trapped object is stuck in the remaining longitude area. A dual-trapped object is a result of the edge-case between a trapped and drifting object: this debris has achieved a stable orbit around both wells. If observed, East-West trapped objects will look as though they are pausing at a well before continuing their drift to the next. Darren S. McKnight and Frank R. Di Pentino analyzed GEO behavior [23] and in their research, developed a criteria capable of determining the risk of a GEO spacecraft to be trapped in a geopotential well. Labelled the $\Delta \Delta$ criteria, the equation is a function of perigee and apogee for the object in question relative to its orbit when decommissioned. The equation is presented as

$$
\Delta \Delta=\left(42164-r_{a}\right)-\left(42164-r_{p}\right)
$$

where $r_{a}$ is the radius as apogee in $\mathrm{km}$ and $r_{p}$ is the radius at perigee in $\mathrm{km}$. It was shown that objects with a $\Delta \Delta$ equal to or lower than $40 \mathrm{~km}$ to be trapped no matter where it was decommissioned in its orbit. The closer an object is to a well in 
longitude during decommissioning however, the easier it is to be trapped by a well. This equation can be illustrated in Figure 1.3.

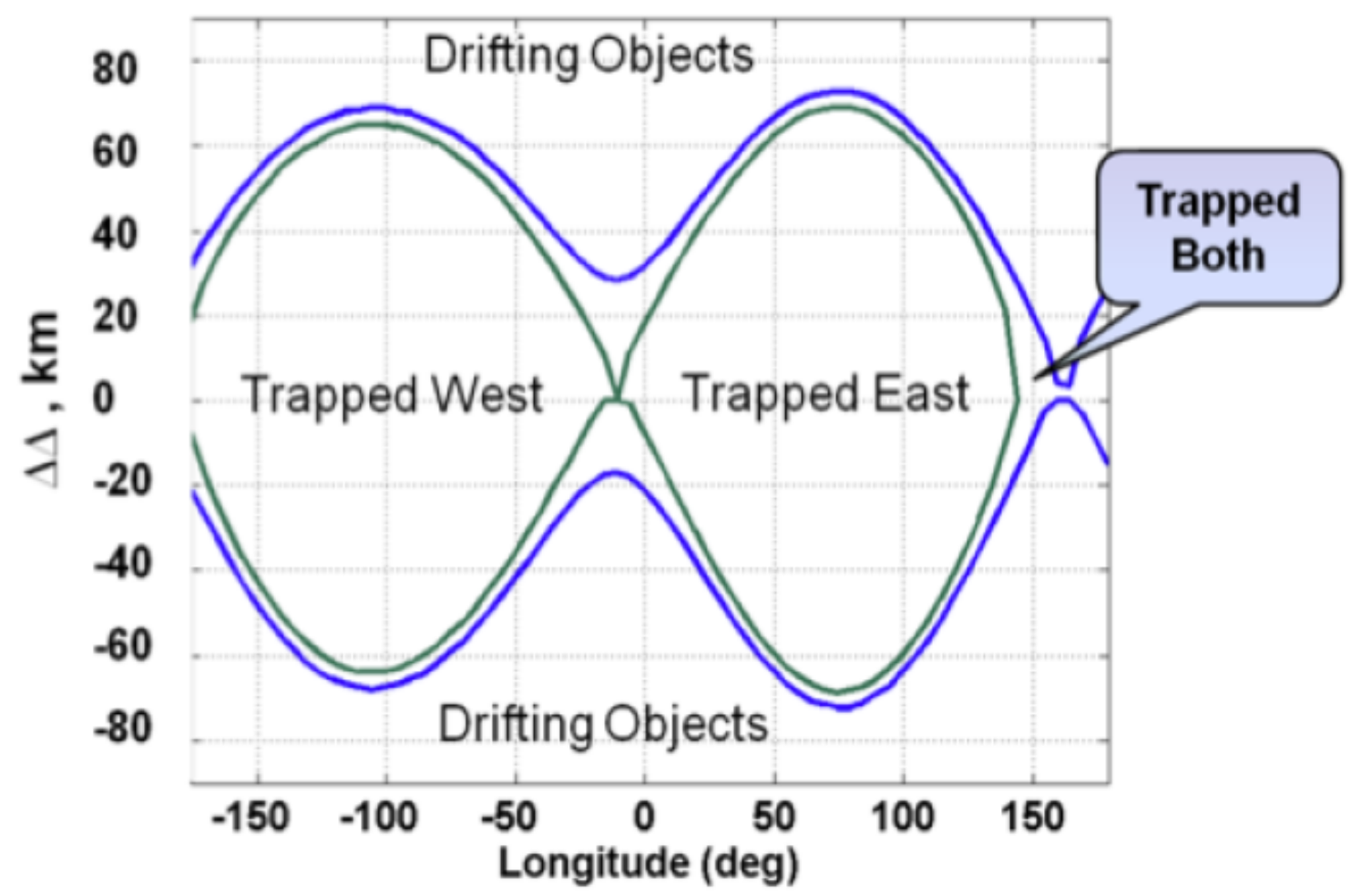

Figure 1.3: Criterion for capture by a geopotential well [23]

\subsection{Two-Line Element Set}

Once observational data for a satellite or piece of debris is processed, its state and orbit can be determined. One common way of representing this determination is with the NORAD two-line element set, or TLE. A TLE is a pair of 69-character lines of data. A TLE is designed to be compact yet contain the information needed to determine a satellite's orbit and location. The TLE appears to a user as follows:

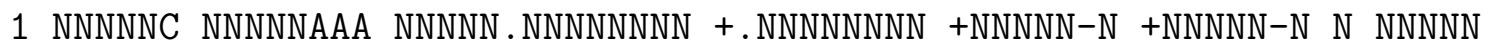

2 NNNNN NNN . NNNN NNN. NNNN NNNNNNN NNN. NNNN NNN. NNNN NN. NNNNNNNNNNNNNNN

where ' $\mathrm{N}$ ' is a number $0-9$, ' $\mathrm{A}$ ' is a character $\mathrm{A}-\mathrm{Z}$ or space, and ' $\mathrm{C}$ ' is a character used to determine the classification of the data (with ' $U$ ' for unclassified, 'S' for 
secret). The data held within these lines can be further defined as given in Table 1.1 and Table $1.2[18]$ :

Table 1.1: First Line of a TLE

\begin{tabular}{|l|l|}
\hline Column & Description \\
\hline 01 & Line Number of Element Data (1) \\
$03-07$ & Satellite Number \\
08 & Classification \\
$10-11$ & International Designator (Last 2 digits of launch year) \\
$12-14$ & International Designator (Launch number of the year) \\
$15-17$ & International Designator (Piece of the launch) \\
$19-20$ & Epoch Year (Last 2 digits of the year) \\
$21-32$ & Epoch (Day) \\
$34-43$ & First Time Derivative of Mean Motion \\
$45-52$ & Second Time Derivative of Mean Motion \\
$54-61$ & BSTAR Drag Term \\
63 & Ephemeris Type \\
$65-68$ & Element Number \\
69 & Checksum (Modulo 10) \\
\hline
\end{tabular}

Table 1.2: Second Line of a TLE

\begin{tabular}{|l|l|}
\hline Column & Description \\
\hline 01 & Line Number of Element Data (2) \\
$03-07$ & Satellite Number \\
$09-16$ & Inclination [deg] \\
$18-25$ & Right Ascension of the Ascending Node [deg] \\
$27-33$ & Eccentricity \\
$35-42$ & Argument of Perigee [deg] \\
$44-51$ & Mean Anomaly [deg] \\
$53-63$ & Mean Motion [rev/day] \\
$64-68$ & Revolution Number at Epoch [rev] \\
69 & Checksum (Modulo 10) \\
\hline
\end{tabular}

A number of sources exist online, such as Celestrak, which provide a historical catalog of TLEs for known and currently tracked satellites [18]. For the purpose of this thesis, historic TLE data was collected from Celestrak for objects of interest. When the historical records are returned, they are parsed in batches for their epoch date as well as the orbital elements contained in the second line of the TLE. This provides a set of dates and orbital elements defining each satellite's orbit and position 
throughout time. Further discussion of the use of TLE-based historic data is provided in chapter 4 .

\subsection{Prior Work in Debris and Debris Modelling}

The foundation of this thesis is built of decades of debris and propagation research. In 1946, before satellites were even placed in orbit around Earth, astronomers such as Fred Whipple were already raising concern for the state of a spacecraft in the face of orbital debris. Whereas Whipple warned of the dangers of meteoroids [29], others like Dr. G. M. Clemence discussed the possibility of collisions that two satellites might face when crossing paths as early as 1954 [4]. Many built upon these discussions, and notably in 1978 Donald J. Kessler proposed what is commonly known as "Kessler syndrome." Kessler syndrome describes a situation where the population of objects in orbit around the Earth is so great that, following the collision of two objects and their resultant debris, there is risk of a runaway cascade effect where additional collision events result. The results of this would be destruction of numerous satellites and the creation of a nearly impenetrable field of debris.

As the use of GEO satellites continued to grow, Jozef C. Van der Ha developed a model in 1986 for the long-term evolution of near-GEO satellites [7]. This model took into account the strongest perturbational forces acting on a simple, two-body orbit to produce results that allowed prediction of spacecraft behavior following endof-life operations. By the 1990's, numerous papers and reports were being published regarding orbital debris and its accurate modelling. A. S. Ganeshan published a 1997 report on the simulation and modelling of debris breakups, and other papers began to follow in suit [10]. Specifically for geosynchronous spacecraft, Westerkamp released a 1997 analysis on space debris risks for GEO satellites outside of the equatorial plane, basing the work on earlier models developed for GEO and LEO spacecraft by Friesen in 1992 [30], [9]. Since then, Nicholas Johnson has argued that the near-GEO satellite characteristics have changed drastically from the classical cases as a result of more complex orbits [17]. These papers have identified not only new ways of viewing and understanding the population of debris and satellites at this regime, but have identified previously unknown and dangerous debris. As a result of NASA's own 
work and the growing need for an accurate model of debris breakup, the LEGEND and EVOLVE breakup models were developed [21]. The LEGEND model is the newer of the two, representing a full-scale model of LEO to GEO model of the debris environment. As the demand for additional satellites and space support rises, work continues from many independent sources to better understand orbital debris and the danger it poses.

\subsection{Thesis Proposal}

The purpose of this thesis is to better understand the unknown behavior of orbital debris in the GEO and graveyard orbits. The first step is determining which forces must be modelled to accurately simulate the debris. To do so, an orbital propagator was created with a high enough accuracy to sufficiently model GEO and graveyardorbit bodies. Additionally, the collision or fragmentation of this debris will be analyzed. This is because, as the debris population grows, there is a growing chance of collisions or further breakup. With geopotential wells effecting debris behavior so heavily, it is worth analyzing whether all fragments of a breakup will be effected in the same way by geopotential wells as its parent objects are. By understanding the behavior of debris in these orbits, we can begin to understand the threat posed to the GEO orbit by uncontrolled debris and what this debris behavior means for future disposal methods of high altitude spacecraft. 


\section{Chapter 2}

\section{Orbital Propagator}

The orbital propagator is responsible for taking an initial state vector and, using a set of equations of motion, carrying out a discrete integration in order to determine a new state vector after a given time interval. The state vector in this case is a position and velocity vector. An orbital propagator was developed to accurately model the behavior of GEO and graveyard-orbit debris under the effects of gravity and perturbative effects.

\subsection{Methods}

There are a variety of numerical propagation methods available for orbital applications, the selection of which is based on ease-of-use, calculation speed, precision capabilities, and limiting cases.

All of the methods presented here are based on the analysis of Keplerian orbits. These orbits are the closed-form solutions of what is known as the two-body problem, an idealized situation where only the Earth and its orbiting body exist. The acceleration of a satellite in the two-body problem is commonly expressed as

$$
\ddot{\vec{r}}=-\mu \frac{\vec{r}}{r^{3}}
$$

where $\vec{r}$ is the position vector of the satellite measured from the center of the central body, and $\mu$ is the body's gravitational parameter. This equation takes into account a few assumptions; namely that the central body is much more massive than the 
satellite, the central body has a symmetric gravitational field, and that the central body is the only body. For for short-term or rough approximations of the satellite's location, these assumptions are all acceptable. For higher-accuracy propagations for these orbits, the acceleration equation needs to take into account the perturbative forces that cause the satellite to deviate from its two-body orbit. Additional information regarding perturbative forces is provided in section 2.2. The perturbative accelerations can all be summed as the force $\vec{p}$ and added to the two-body equation as

$$
\ddot{\vec{r}}=-\mu \frac{\vec{r}}{r^{3}}+\vec{p}
$$

In almost all cases, the perturbations will be orders of magnitude weaker than the basic two-body gravity. The major exception to this is drag at low altitudes, which can rapidly degrade an orbit and cause reentry. Adding perturbations to the acceleration in this form is the most common method, but there are other techniques that may be better suited for the situation.

\subsubsection{Cowell's Method}

Cowell's Method was developed by British astronomer Philip H. Cowell as a direct numerical integration method capable of integrating the equations of motion of an orbiting body. It can account for perturbations acting on the orbiting body, but it does not easily handle perturbations significantly smaller than the primary acceleration. This method is simple to implement from a coding perspective, but it requires more computational power and can fail to accurately model minor perturbations over long time spans. The equation of motion for a perturbed two-body orbit can be expressed with Cowell's method as in Equation 2.2. When considering a satellite orbiting the

Earth, the equation of motion $\ddot{\vec{r}}$ is in terms of the gravitational parameter for Earth $\mu$, the position vector of the spacecraft from the center of Earth $\vec{r}$, and the sum of the perturbing accelerations is $\vec{p}$. 


\subsubsection{Encke's Method}

A method developed by Johann Franz Encke in the 19th century, Encke's method can be used for two-body propagations featuring perturbations. The method looks at a two-body osculating orbit as a reference orbit, adding on a perturbative deviation to the orbit which results in the perturbed location as in Figure 2.1. This process

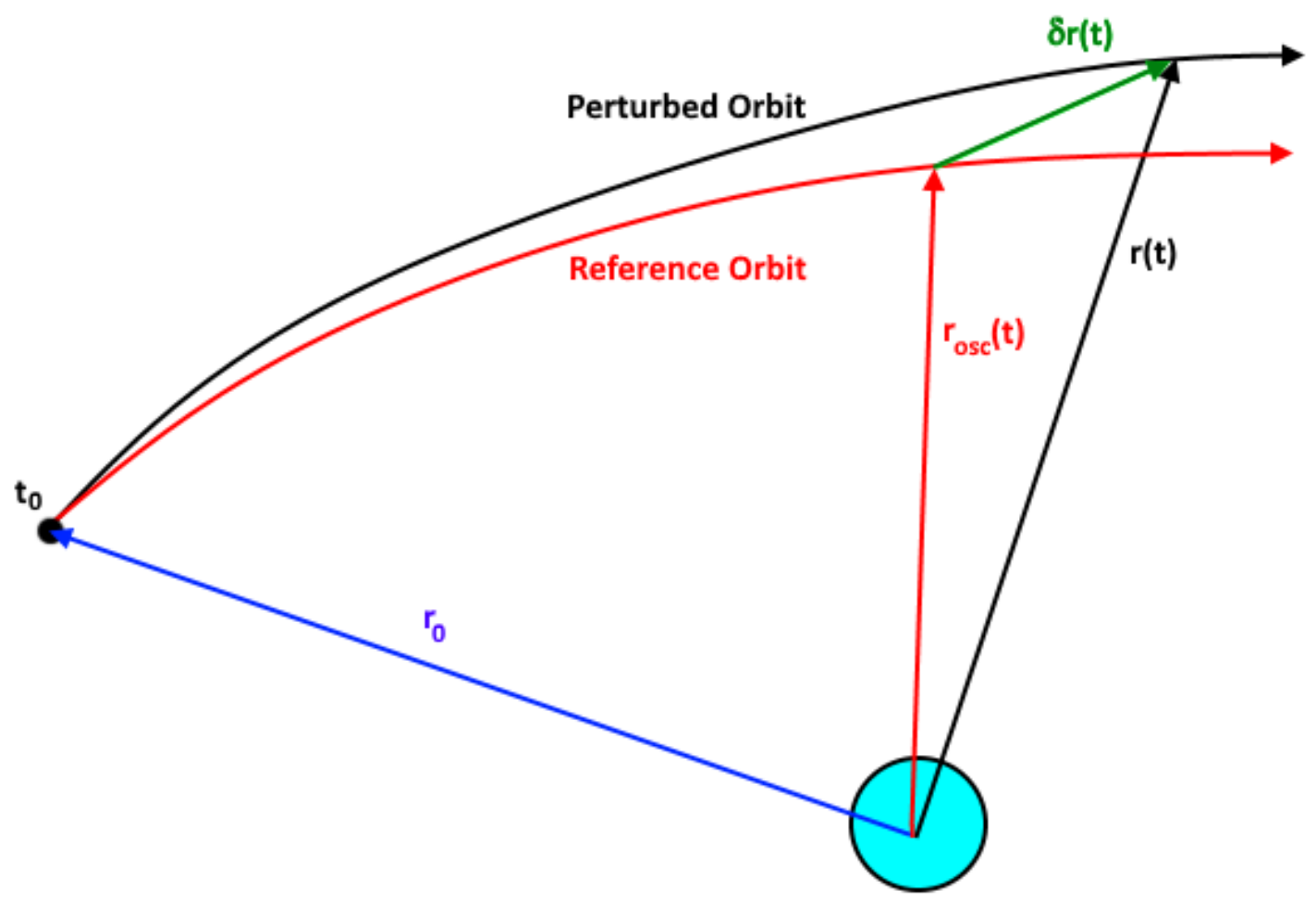

Figure 2.1: Osculating and Reference Orbits of Encke's Method

is then repeated continuously, resulting in an approximation of the perturbed orbit trajectory. This method is capable of taking large step sizes in certain scenarios and is fitting for usage in interplanetary trajectories. In terms of disadvantages, it is a complex method to implement and requires additional computation if perturbations are minute compared to the reference orbit. Encke's Method can be represented with two equations, 2.3 for the two-body acceleration and 2.4 for the perturbed acceleration. These are then used to find the perturbed orbit in 2.5

$$
\ddot{\vec{r}}=-\frac{\mu}{r^{3}} \vec{r}
$$




$$
\begin{gathered}
\delta \ddot{\vec{r}}=\vec{p}+\frac{\mu}{r^{3}}\left\{\left(1-\frac{r^{3}}{r_{p}^{3}}\right) \overrightarrow{r_{p}}-\delta \vec{r}\right\} \\
\overrightarrow{r_{p}}=\vec{r}+\delta \vec{r}
\end{gathered}
$$

\subsubsection{Variation of Parameters Method}

The Variation of Parameters (VoP) Method is an approach that seeks to address the accuracy issues of other propagation methods. Whereas the other methods discussed utilize a state vector comprised of the position and velocity vectors of the orbiting body, VoP tracks the perturbative effects of the orbit using a number of elements that define the orbit and location of the orbiting body along that orbit. It is a higher accuracy propagation method than either Cowell's or Encke's Method as the variation in the elements is orders of magnitude closer to the elements themselves than the variation in the state vector is to any given position and velocity.

While VoP is capable of higher accuracy propagations, it is difficult to implement and computationally expensive. Additionally, there are various orbital element sets that can be implemented with this method and must be chosen to fit the scenario being analyzed. A small selection will be presented here but a full survey is given by Hintz [14].

\subsubsection{True and Modified Classical Element Set}

The true classical element set is composed of the semimajor axis, eccentricity, inclination, argument of periapsis, right ascension of the ascending node, and time of perifocal passage. These six elements can be used to describe the orbit and the location of the satellite along it. Perhaps more familiar is the modified classical element set, displayed in Table 2.1, which replaces the time of perifocal passage with true anomaly or mean anomaly.

Both forms are singular at eccentricities of 0 and 1, meaning they cannot be used for circular or parabolic orbits. Additionally, they are singular at inclinations of 0 
and $\pi$. For this reason, they are not suited for the circular or near-circular orbits of GEO regimes and debris.

Table 2.1: Modified Classical Element Set

\begin{tabular}{|c|l|}
\hline Element & Modified Classical \\
\hline $\mathbf{1}$ & a (semimajor axis) \\
\hline $\mathbf{2}$ & e (eccentricity) \\
\hline $\mathbf{3}$ & i (inclination) \\
\hline $\mathbf{4}$ & $\omega$ (argument of periapsis) \\
\hline $\mathbf{5}$ & $\Omega$ (right ascension of the ascending node \\
\hline $\mathbf{6}$ & $\theta$ (true anomaly) or M (mean anomaly) \\
\hline Notes & Singular at $e=0,1 ; i=0, \pi$. \\
\hline
\end{tabular}

\subsubsection{Equinoctial Element Set}

The equinoctial element set is composed of 6 elements created from the classical orbital elements and listed in Table 2.2. This set has the benefit of only remaining undefined for parabolic and hyperbolic orbits, however the element set is defined differently for posigrade and retrograde orbits [3].

Table 2.2: Equinoctial Element Set

\begin{tabular}{|c|l|c|}
\hline Element & Equinoctial, Posigrade & Equinoctial, Retrograde \\
\hline $\mathbf{1}$ & $\mathrm{a}$ & $\mathrm{a}$ \\
\hline $\mathbf{2}$ & $h=e * \sin (\omega+\Omega)$ & $h=e * \sin (\omega-\Omega)$ \\
\hline $\mathbf{3}$ & $k=e * \cos (\omega+\Omega)$ & $k=e * \cos (\omega-\Omega)$ \\
\hline $\mathbf{4}$ & $p=\tan (i / 2) * \sin (\Omega)$ & $p=\cot (i / 2) * \sin (\Omega)$ \\
\hline $\mathbf{5}$ & $q=\tan (i / 2) * \cos (\Omega)$ & $q=\cot (i / 2) * \cos (\Omega)$ \\
\hline $\mathbf{6}$ & $\lambda=M+\omega+\Omega$ & $\lambda=M+\omega-\Omega$ \\
\hline Notes & Undefined for $e \geqslant 1$. & Undefined for $e \geqslant 1$. \\
\hline
\end{tabular}

\subsubsection{Modified Equinoctial Element Set}

A modified set of the equinoctial element set, this set uses 6 elements to define an orbiting bodies position and unlike any previously mentioned set, it is nonsingular for all eccentricities and inclinations [14]. A retrograde factor is used to define the set 
of elements differently depending on whether a posigrade or retrograde orbit is being examined. This set is shown in Table 2.3.

Table 2.3: Modified Equinoctial Element Set

\begin{tabular}{|c|l|}
\hline Element & Modified Equinoctial \\
\hline $\mathbf{1}$ & $p$ (semilatus rectum $)$ \\
\hline $\mathbf{2}$ & $f=e * \cos (\omega+I \Omega)$ \\
\hline $\mathbf{3}$ & $g=e * \sin (\omega+I \Omega$ \\
\hline $\mathbf{4}$ & $h=\tan ^{I}(i / 2) * \cos \Omega$ \\
\hline $\mathbf{5}$ & $k=\tan ^{I}(i / 2) * \sin \Omega$ \\
\hline $\mathbf{6}$ & $L=\omega+I \Omega+\theta$ \\
\hline Notes & $\mathrm{I}=+1$ for posigrade, I $=-1$ for retrograde \\
\hline
\end{tabular}

\subsubsection{Chosen Approach}

This thesis builds upon previous work conducted by Christina Diaz in her Cal Poly thesis on the propagation and collision of GEO debris [8]. In this work the Cowell's Method was chosen as it was a simple method to implement and did not provide any limitations on operating cases. However, an accurate propagation model could not be created and this was assumed to be due to the accuracy limitations of Cowell's Method. This thesis will test that hypothesis, recreating a Cowell Method propagator and a VoP propagator and testing both to see where limitations arise. In order to avoid any limiting situations, the modified equinoctial element set was chosen for the VoP propagator.

While the Cowell Propagator is straight forward and already discussed, the Equinoctial propagator requires additional work. In order to integrate the set of VoP elements, the derivatives of each element must be calculated at each time step. The equations of motion for the modified equinoctial elements are as described in Eq. 2.6-2.11,

$$
\begin{gathered}
d p=\sqrt{\frac{p}{\mu}} *\left(\frac{2 p C}{w}\right) \\
d f=\sqrt{\frac{p}{\mu}} *\left[S * \sin (L)+\frac{C}{w}((w+1) \cos (L)+f)-\frac{N g}{w}(h \sin (L)-k \cos (L))\right]
\end{gathered}
$$




$$
\begin{aligned}
& d g=\sqrt{\frac{p}{\mu}} *\left[-S * \cos (L)+\frac{C}{w}((w+1) \sin (L)+g)+\frac{N f}{w}(h \sin (L)-k \cos (L))\right] \\
& d h=\sqrt{\frac{p}{\mu}} *\left(\frac{s^{2} N}{2 w} \cos (L)\right) \\
& d k=\sqrt{\frac{p}{\mu}} *\left(\frac{s^{2} N}{2 w} \sin (L)\right) \\
& d L=\sqrt{\frac{p}{\mu}} * \frac{N}{w}(h \sin (L)-k \cos (L))+\sqrt{p \mu}\left(\frac{w}{p}\right)^{2}
\end{aligned}
$$

with Auxiliary Variables

$$
\begin{gathered}
s=1+h^{2}+k^{2} \\
w=1+f \cos (L)+g \sin (L) .
\end{gathered}
$$

The S, C, and $\mathrm{N}$ variables are the perturbing acceleration components in the directions perpendicular to the radius vector in the direction of motion, along the radius vector outward, and normal to the orbital plane in the direction of the angular momentum vector, respectively [14]. These components map to the RSW satellite coordinate system, with $\mathrm{S}, \mathrm{C}$, and $\mathrm{N}$ equivalent to R, S, and W. The RSW frame will be discussed further in section 2.2.

\subsection{Perturbation Analysis}

For the purpose of debris propagation in GEO, each method will additionally have to integrate the strongest perturbatory effects of the GEO regime. The strongest perturbations to the simple two-body Earth-Satellite system comes from the aspherocity of Earth, the gravitational effects of the Sun and Moon, and solar radiation pressure. There are a number of other effects that follow these that, for even higher-accuracy applications, may need to be included in a perturbation analysis for the GEO regime. These additional effects were not included in the orbital propagators at the center of 
Table 2.4: Perturbation Order of Influence

\begin{tabular}{|l|l|l|}
\hline Perturbation & Order $(\mathbf{m} / \mathbf{s})$ & Notes \\
\hline Geopotential (J2) & $10^{-5}$ & - \\
\hline Geopotential (J22) & $10^{-7}$ & Nearly negligible for all but GEO. \\
\hline Geopotential (All others) & $10^{-7}$ & $\begin{array}{l}\text { Cumulative effect of all other } \\
\text { terms. }\end{array}$ \\
\hline Drag & $0(\mathrm{GEO})$ & No effect at GEO altitudes. \\
\hline Sun Gravity & $10^{-6}$ & - \\
\hline Lunar Gravity & $10^{-4}$ & - \\
\hline Solar Radiation Pressure & $10^{-7}$ & $\begin{array}{l}\text { Zero when eclipsed. Depen- } \\
\text { dant on material properties, area, } \\
\text { mass. }\end{array}$ \\
\hline Earth Radiation Pressure & $10^{-8}$ & $\begin{array}{l}\text { Radiation re-emitted by Earth. } \\
\text { Affects LEO by 10-35 percent of } \\
\text { SRP. }\end{array}$ \\
\hline Earth Tides & $10^{-9}$ & $\begin{array}{l}\text { Ocean Tides are an order weaker } \\
\text { than Solid Earth Tides. }\end{array}$ \\
\hline Relativistic Effects & - & $\begin{array}{l}\text { General effects on order of 1cm } \\
\text { of deviation. Solar relativistic ef- } \\
\text { fects on order of 0.1mm of devi- } \\
\text { ation. }\end{array}$ \\
\hline
\end{tabular}

this thesis but are included in this section for completeness. A break down of the average order of effect that each perturbation has on the acceleration of a spacecraft is provided in Table 2.4 .

Before being used with the equinoctial VoP method, the perturbative accelerations must be broken down into $\mathrm{S}, \mathrm{C}$, and $\mathrm{N}$ components. This is done with the $\mathrm{r}$, s, and $\mathrm{w}$ unit vectors that express the RSW satellite coordinate system in the following equations. In these, $\vec{r}$ and $\vec{v}$ represent the position and velocity vectors of the spacecraft, respectively.

$$
\begin{gathered}
\hat{u}_{r}=\frac{\vec{r}}{\|\vec{r}\|} \\
\hat{u}_{w}=\frac{\vec{r} \times \vec{v}}{\|\vec{r} \times \vec{v}\|} \\
\hat{u}_{s}=\frac{\hat{u}_{w} \times \hat{u}_{r}}{\left\|\hat{u}_{w} \times \hat{u}_{r}\right\|}
\end{gathered}
$$


For the perturbative acceleration $\vec{p}$ this becomes

$$
\begin{aligned}
& C=\vec{p} \cdot \hat{u}_{r} \\
& S=\vec{p} \cdot \hat{u}_{s} \\
& N=\vec{p} \cdot \hat{u}_{w}
\end{aligned}
$$

\subsubsection{Earth Oblateness}

One of the basic assumptions made when modelling the two-body orbital dynamics of an Earth-centered spacecraft is the assumption that Earth is a perfect sphere with a symmetrical mass distribution. In reality, the Earth and other celestial bodies are closer to oblate spheroids in shape, and this deviation is responsible for a perturbative effect from the basic, symmetric-spherical gravitational model. Additionally, localized high-mass anomalies as a result of mountain ranges and continents result in a further deviation from the basic model. These variations are accounted for using a set of spherical harmonic expansions.

Zonal harmonics can be viewed as a set of different expansions that break up the mass distribution effects by latitude, sectoral harmonics break the Earth effects into longitude ranges, and tesseral harmonics result in a checkerboard-style analysis of the mass distribution. These are shown in Figure 2.2.

Oblateness effects are typically discussed with a $J_{m n}$ notation, where $\mathrm{m}$ is always greater than or equal to $\mathrm{n}$. When $n=0$ zonal harmonics are being represented, when $m=n$ sectorial harmonics are being represented, and when $m \neq n$ then tesseral harmonics are represented. The acceleration effects considered most often are also the largest of terms, the $J_{2}, J_{3}$, and $J_{22}$ terms. The $J_{2}$ term is the strongest of all oblateness terms and is stronger in most cases than any other perturbation, except perhaps drag on very low-orbit satellites. The $J_{3}$ term results in roughly a thousand times weaker of a perturbation, and the $J_{22}$ is even weaker. Due to the position of the 

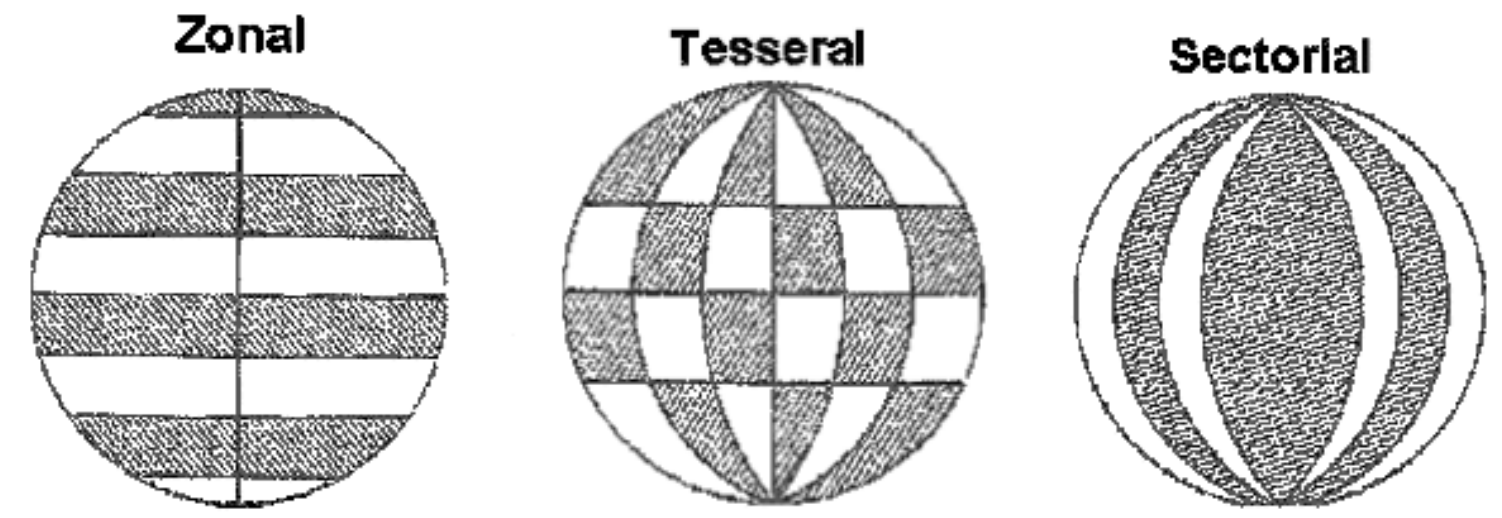

Figure 2.2: The three types of spherical harmonics

GEO orbit however, the $J_{22}$ term is stronger in this regime than $J_{3}$. When talking about GEO spacecraft and geopotential wells, this paper will show that terms up to $J_{33}$ must be considered.

To take these oblateness terms into account, the perturbative acceleration is presented as the negative gradient of the gravitational potential energy per unit mass of the earth in Cartesian coordinates,

$$
\vec{p}=-\vec{\nabla} U=-\frac{\delta U}{\delta x} \hat{\mathbf{I}}-\frac{\delta U}{\delta y} \hat{\mathbf{J}}-\frac{\delta U}{\delta z} \hat{\mathbf{K}}
$$

\subsubsection{1 $\quad J_{2}$ Perturbation}

The $J_{2}$ term is the largest of the perturbations because it breaks the Earth into 3 latitude ranges. Because the Earth features a prominent bulge of mass at its equatorial latitudes, this mass distribution is best accounted for in this zonal harmonic.

To further break down $U$ for a zonal harmonic expansion,

$$
U(r, \phi)=\frac{\mu}{r} \sum_{m=2}^{\infty} J_{m}\left(\frac{R_{\oplus}}{r}\right)^{m} P_{m} \cos (\phi) .
$$

Here, $\mu$ is the gravitational parameter of Earth, or $\mu=G M$ with $G$ as the universal gravitational constant and $M$ as the mass of the Earth. $r$ is the position of the satellite from the center of Earth, $J_{m}$ is the zonal harmonic, $R$ is the equatorial 
radius of the Earth, $P_{k}$ is the relevant Legendre polynomial for the zonal harmonic, and $\phi$ is represented as

$$
\phi=\tan ^{-1} \frac{\sqrt{x^{2}+y^{2}}}{z}
$$

with $\mathrm{x}, \mathrm{y}$, and $\mathrm{z}$ being the distance of the satellite to the Earth's center in the I, J, and $\mathrm{K}$ directions of the Cartesian frame accordingly. $P_{k}$ and the $J_{m}$ terms can be found in texts such as Vallado and Curtis [35], [6].

The acceleration of the $J_{2}$ term is provided in a simplified form in the following equation:

$$
\vec{p}=\frac{3 J_{2} \mu R^{2}}{2 r^{4}}\left[\frac{x}{r}\left(5 \frac{z^{2}}{r^{2}}-1\right) \hat{\mathbf{I}}+\frac{y}{r}\left(5 \frac{z^{2}}{r^{2}}-1\right) \hat{\mathbf{J}}+\frac{z}{r}\left(5 \frac{z^{2}}{r^{2}}-3\right) \hat{\mathbf{K}}\right] .
$$

\subsubsection{2 $J_{22}$ Perturbation}

The $J_{22}$ term, also refered to as triaxiality, is a major perturbatory source for GEO orbits as a result of a resonant effect [32]. As a sectoral harmonic, it is clear why this term has such a great effect on GEO orbits, as GEO satellites are places into specific longitude bins. Analysis of sectoral harmonics splits the Earth into longitude bands which help exhibit the gravitational effects on satellites placed within these bands. Furthermore, the Earth not only features an equatorial bulge that contributes to the $J_{2}$ term being so strong, but the Earth is also slightly elliptical when viewed down its polar axis. As a result of this, $J_{22}$ has a very noticeable effect on GEO spacecraft. The acceleration caused by this perturbative force is calculated using the following

expressions, where $\phi$ is considered the geocentric latitude and expressed differently than in the previous $J_{2}$ term, and $\lambda$ is the geocentric longitude:

$$
\begin{gathered}
\phi=\tan ^{-1}\left(\frac{z}{\sqrt{x^{2}+y^{2}}}\right) \\
\lambda=\tan ^{-1}\left(\frac{y}{x}\right)
\end{gathered}
$$

The perturbative acceleration will be broken down into its three components for the Cartesian coordinate system. As the gravitational perturbation acceleration is 
a gradient of $\mathrm{U}$, this will be further broken down and each term will be handled individually.

$$
\begin{aligned}
& \vec{p}=\left[p_{i}, \quad p_{j}, \quad p_{k}\right] \\
& p_{i}=\frac{\delta U}{\delta r} \frac{\delta r}{\delta x}+\frac{\delta U}{\delta \phi} \frac{\delta \phi}{\delta x}+\frac{\delta U}{\delta \lambda} \frac{\delta \lambda}{\delta x} \\
& p_{j}=\frac{\delta U}{\delta r} \frac{\delta r}{\delta y}+\frac{\delta U}{\delta \phi} \frac{\delta \phi}{\delta y}+\frac{\delta U}{\delta \lambda} \frac{\delta \lambda}{\delta y} \\
& p_{k}=\frac{\delta U}{\delta r} \frac{\delta r}{\delta z}+\frac{\delta U}{\delta \phi} \frac{\delta \phi}{\delta z}+\frac{\delta U}{\delta \lambda} \frac{\delta \lambda}{\delta z}
\end{aligned}
$$

From here, each derivative of $U$ is calculated

$$
\begin{gathered}
\frac{\delta U}{\delta r}=\frac{-9 \mu R_{\oplus}^{2}}{r^{4}} \cos ^{2}(\phi)\left[C_{22} \cos (2 \lambda)+S_{22} \sin (2 \lambda)\right] \\
\frac{\delta U}{\delta \phi}=\frac{-6 \mu R_{\oplus}^{2}}{r^{3}} \sin (\phi) \cos (\phi)\left[C_{22} \cos (2 \lambda)+S_{22} \sin (2 \lambda)\right] \\
\frac{\delta U}{\delta \lambda}=\frac{6 \mu R_{\oplus}^{2}}{r^{3}} \cos ^{2}(\phi)\left[S_{22} \cos (2 \lambda)-C_{22} \sin (2 \lambda)\right]
\end{gathered}
$$

In these equations, As is each term of $r, \phi$, and $\lambda$

$$
\begin{gathered}
\nabla \vec{r}=\left[\frac{\delta r}{\delta x}, \frac{\delta r}{\delta y}, \frac{\delta r}{\delta z}\right]=\left[\frac{x}{r}, \frac{y}{r}, \frac{z}{r}\right] \\
\nabla \vec{\phi}=\left[\frac{\delta \phi}{\delta x}, \frac{\delta \phi}{\delta y}, \frac{\delta \phi}{\delta z}\right]=\left[\frac{-x z}{r^{3} \cos \phi}, \frac{-y z}{r^{3} \cos \phi}, \frac{\cos \phi}{r}\right] \\
\nabla \vec{\lambda}=\left[\frac{\delta \lambda}{\delta x}, \frac{\delta \lambda}{\delta y}, \frac{\delta \lambda}{\delta z}\right]=\left[\frac{-y}{x^{2}+y^{2}}, \frac{x}{x^{2}+y^{2}}, 0\right]
\end{gathered}
$$

These equations can be multiplied and summed together to arrive at a simplified equation as given in Section 2.2.1.1. The final resultant acceleration is then rotated 
into the RSW frame and added into the $\mathrm{S}, \mathrm{C}$, and N values of the equinoctial elements prior to calculating the derivatives of the equinoctial element set in the propagator.

\subsubsection{Additional Geopotential Perturbations}

While the $J_{2}$ and $J_{22}$ terms have been expressed here directly, another method exists if a large number of geopotential terms needs to be considered [35]. Rather than solving for the solution of each term individually, terms are summed up from the $J_{2}$ term up to the term of interest, as shown in the following equations.

$$
\begin{gathered}
\frac{\delta U}{\delta r}=-\frac{\mu}{r^{2}} \sum_{m=2}^{\infty} \sum_{n=0}^{m}\left(\frac{R_{\oplus}}{r}\right)^{m}(m+1) P_{m, n}\left\{C_{m, n} \cos (n \lambda)+S_{m, n} \sin (n \lambda)\right\} \\
\frac{\delta U}{\delta \phi}=-\frac{\mu}{r} \sum_{m=2}^{\infty} \sum_{n=0}^{m}\left(\frac{R_{\oplus}}{r}\right)^{m}\left\{P_{m, n+1}-n \tan (\phi) P_{m, n}\right\}\left\{C_{m, n} \cos (n \lambda)+S_{m, n} \sin (n \lambda)\right\} \\
\frac{\delta U}{\delta \lambda}=-\frac{\mu}{r} \sum_{m=2}^{\infty} \sum_{n=0}^{m}\left(\frac{R_{\oplus}}{r}\right)^{m} m P_{m, n}\left\{S_{m, n} \cos (n \lambda)-C_{m, n} \sin (n \lambda)\right\}
\end{gathered}
$$

With these three terms, the $P_{m, n}$ terms are expressed using $P_{k}[\sin (\phi)]$ which is contrary to the earlier definition supplied. This form and its expressions are provided in Vallado [35]. At this point they can be plugged into the following acceleration terms. These acceleration terms are the perturbative force broken down into its I, J, and K components.

$$
\begin{gathered}
a_{I}=\left\{\frac{1}{r} \frac{\delta U}{\delta r}-\frac{r_{K}}{r^{2} \sqrt{r_{I}^{2}+r_{J}^{2}}} \frac{\delta U}{\delta \phi}\right\} r_{I}-\left\{\frac{1}{r_{I}^{2}+r_{J}^{2}} \frac{\delta U}{\delta \lambda}\right\} r_{J} \\
a_{J}=\left\{\frac{1}{r} \frac{\delta U}{\delta r}-\frac{r_{K}}{r^{2} \sqrt{r_{I}^{2}+r_{J}^{2}}} \frac{\delta U}{\delta \phi}\right\} r_{J}+\left\{\frac{1}{r_{I}^{2}+r_{J}^{2}} \frac{\delta U}{\delta \lambda}\right\} r_{I} \\
a_{K}=\frac{1}{r} \frac{\delta U}{\delta r} r_{K}+\frac{\sqrt{r_{I}^{2}+r_{J}^{2}}}{r^{2}} \frac{\delta U}{\delta \phi}
\end{gathered}
$$




\subsubsection{Solar Body Effects}

For satellites within Earth's orbit, we can consider the gravity exerted by the Sun as a perturbation on the base orbit. The perturbative acceleration is presented as

$$
\vec{p}=\mu \odot\left(\frac{\vec{r} \odot / s}{r_{\odot / s}^{3}}-\frac{\vec{r} \odot}{r_{\odot}^{3}}\right)
$$

where the subscript $\odot$ is used to represent the Sun variables for gravitational parameter and position measured from the Earth's center, and $r \odot / s$ is the position of the sun relative to the satellite. A visualization of these vectors is provided in Figure 2.3.

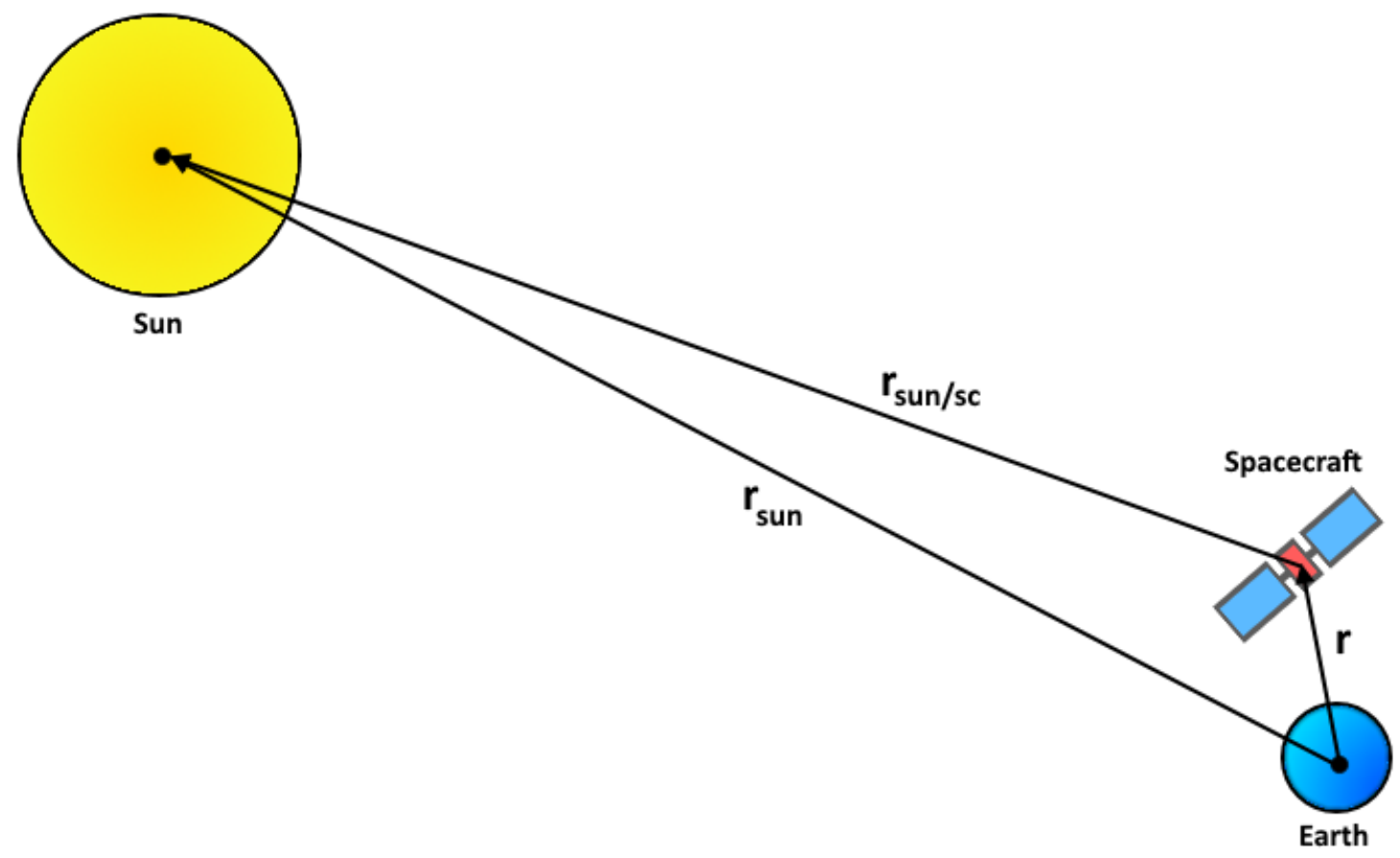

Figure 2.3: Vectors for Solar Body Effects

The use of this formulation results in the subtraction of two very similar numbers. To avoid the issue this will cause in computer-based numerical computations, the equation can be rewritten using an alternative formulation [2]:

$$
\vec{p}=\frac{\mu \odot}{r_{\odot / s}^{3}}\left[F(q) \overrightarrow{r_{\odot}}-\vec{r}\right]
$$




$$
\begin{gathered}
F(q)=\frac{q^{2}-3 q+3}{1+(1-q)^{\frac{3}{2}}} q \\
q=\frac{\vec{r} \cdot\left(2 \vec{r}_{\odot}-\vec{r}\right)}{r_{\odot}^{2}}
\end{gathered}
$$

To find the location of the Sun relative to the Earth we need to know three values: the obliquity of the ecliptic $\epsilon$, the longitude of the Sun $\lambda$, and the geocentric position vector of the sun $\vec{r}_{\odot}$. The longitude is given in degrees as

$$
\lambda=L+1.915 \sin (M)+0.0200 \sin (2 M)
$$

where lambda is constrained between 0 and 360 degrees. L and M, the mean longitude and the mean anomaly of the sun respectively, are calculated in degrees as

$$
\begin{gathered}
L=280.459+0.98564736 n \\
M=357.529+0.98560023 n
\end{gathered}
$$

where both are constrained between 0 and 360 degrees. $n$ is the number of days since J2000, or in days,

$$
n=J D-2451545.0 \text {. }
$$

Using $n$, the obliquity can also be calculated:

$$
\epsilon=23.439-3.56\left(10^{-7}\right) n .
$$

The distance of the Sun to the Earth is calculated in Astronomical Units as

$$
r_{\odot}=1.00014-0.01671 \cos (M)-0.000140 \cos (2 M)
$$


and finally the $\vec{r}_{\odot}$ vector can be calculated as

$$
\vec{r}_{\odot}=r_{\odot}[\cos (\lambda) \hat{\mathbf{I}}+\sin (\lambda) \cos (\epsilon) \hat{\mathbf{J}}+\sin (\lambda) \sin (\epsilon) \hat{\mathbf{K}}]
$$

\subsubsection{Lunar Body Effects}

Similar to the solar body effects, the lunar gravity can be expressed simply as a perturbation to the base orbit of an Earth-centered spacecraft. The perturbative acceleration is presented as

$$
\vec{p}=\mu_{m}\left(\frac{\vec{r}_{m / s}}{r_{m / s}^{3}}-\frac{\vec{r}_{m}}{r_{m}^{3}}\right)
$$

where the subscript $m$ is used to express terms related to the moon. The vector $\vec{r}_{m}$ is calculated from the Earth's center to the moon's center, and the vector $\vec{r}_{m / s}$ is the vector to the moon's center from the spacecraft.

$$
\vec{r}_{m / s}=\vec{r}_{m}-\vec{r}_{s}
$$

A visualization of these vectors is provided in Figure 2.4.

For the calculation of this perturbation, the position of the moon relative to the Earth must be known. This is calculated using the formulas presented in The Astronomical Almanac for the lunar ecliptic longitude $\lambda$, lunar ecliptic latitude $\delta$, and lunar horizontal parallax HP [27]. These are approximate values based on the Julian

date of analysis but are accurate enough for the needs of this thesis. The geocentric equatorial position of the moon is calculated as

$$
\vec{r}_{m}=\left\{\begin{array}{l}
r_{m} \cos (\delta) \cos (\lambda) \hat{\mathbf{I}} \\
r_{m}(\cos (\epsilon) \cos (\delta) \sin (\lambda)-\sin (\epsilon) \sin (\delta)) \hat{\mathbf{J}} \\
r_{m}(\sin (\epsilon) \cos (\delta) \sin (\lambda)+\cos (\epsilon) \sin (\delta)) \hat{\mathbf{K}}
\end{array}\right\}
$$




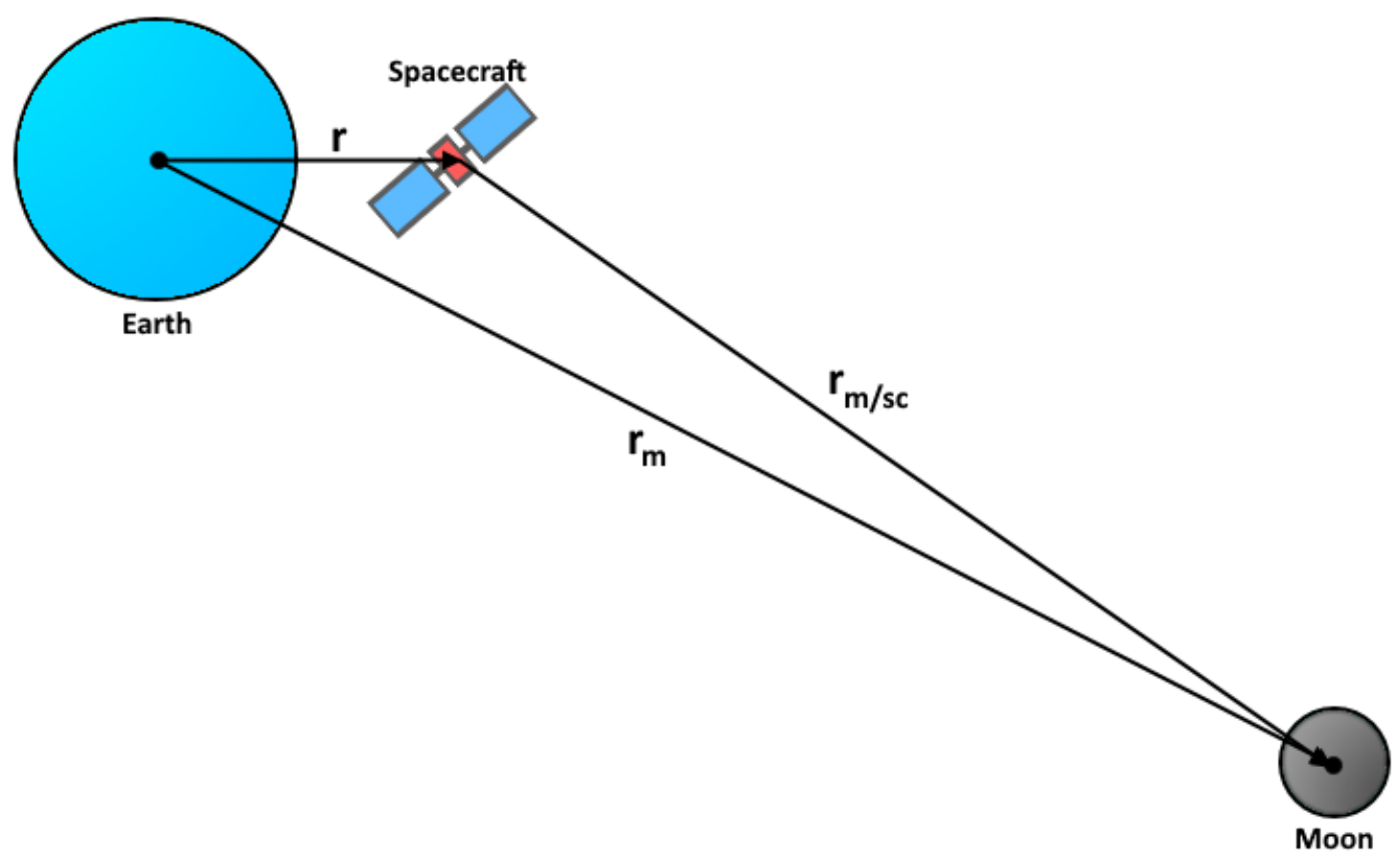

Figure 2.4: Vectors for Lunar Body Effects

where $\epsilon$ is the obliquity of the ecliptic. The distance of the moon $r_{m}$, is provided as

$$
r_{m}=\frac{R_{E}}{\sin (H P)}
$$

with $R_{E}$ as the Earth's equatorial radius and $\mathrm{HP}$ as the horizontal parallax. The lunar ecliptic longitude $\lambda$ and the lunar ecliptic latitude $\delta$ are calculated in degrees and constrained between 0 and 360 :

$$
\begin{gathered}
\lambda=b_{0}+c_{0} T_{0}+\sum_{i=1}^{6} a_{i} \sin \left(b_{i}+c_{i} T_{0}\right) \\
\delta=\sum_{i=1}^{4} d_{i} \sin \left(e_{i}+f_{i} T_{0}\right)
\end{gathered}
$$

The horizontal parallax is provided as

$$
H P=g_{0} \sum_{i=1}^{4} g_{i} \cos \left(h_{i}+k_{i} T_{0}\right)
$$


and is constrained between 0 and 180 degrees. All three of these equations require the calculation of $T_{0}$, the number of Julian centuries since J2000 where JD is is the current Julian day:

$$
T_{0}=\frac{J D-2451545.0}{36525}
$$

Finally, the obliquity of the moon ecliptic is provided in degrees as

$$
\epsilon=23.439-0.0130042 T_{0}
$$

The coefficients $a$ through $k$ and their subscripts provided in the equations above can be found in Battin or the Astronomical Almanac [2], [27].

\subsubsection{Solar Radiation Pressure}

Solar Radiation Pressure (SRP) is a non-conservative force caused by photons expelled by the Sun interacting and exerting pressure on objects in orbit. These photons bounce off of the areas of the spacecraft exposed to the sun. For a more reflective spacecraft, a larger number of photons will bounce off of the spacecraft instead of being absorbed as heat. When this happens, energy is imparted on the spacecraft exerting not only a translational force, but a rotational acceleration when the force is not symmetrically exerted around the spacecraft's center of mass. The effects of this perturbation are on the order of $10^{-7} \mathrm{~m} / \mathrm{s}^{2}$ but can become drastically greater for spacecraft with a large surface area or a more reflective surface material. Additionally, this force is strongest at higher altitudes.

The perturbative acceleration due to SRP can be expressed as

$$
\vec{p}=-p_{S R} \hat{u}
$$

where $\hat{u}$ is the unit vector pointing from the satellite towards the Sun and the perturbative magnitude is

$$
p_{S R}=\nu \frac{S}{c} \frac{C_{R} A}{m}
$$

$\nu$ is the shadow function, a 0 when the satellite is in Earth's shadow and 1 when 
the satellite is in view of the sun. $\frac{S}{c}$ is the Solar constant over the speed of light, while $A$ is the cross sectional area of the spacecraft exposed to the Sun and $C_{R}$ is the coefficient of reflectivity for this area. The use of the shadow function allows the force to be calculated as zero when the satellite is eclipsed by the Earth, but as with the solar and lunar gravity perturbations, this means the position of the Sun relative to the Earth and the spacecraft must be tracked.

The shadow function can be expressed as follows:

$$
\begin{aligned}
& \theta=\cos ^{-1}\left(\frac{\vec{r}_{\odot} \cdot \vec{r}_{s}}{\left\|\vec{r}_{\odot} \vec{r}_{s}\right\|}\right) \\
& \theta_{1}=\cos ^{-1}\left(\frac{r_{\oplus}}{\left\|\vec{r}_{s}\right\|}\right) \\
& \theta_{2}=\cos ^{-1}\left(\frac{r_{\oplus}}{\left\|\vec{r}_{\odot}\right\|}\right)
\end{aligned}
$$

$$
\text { If } \theta_{1}+\theta_{2}<\theta \text {, then } \nu=0
$$

$$
\text { If } \theta_{1}+\theta_{2}>\theta \text {, then } \nu=1
$$

Figure 2.5 displays these angles with reference to the Earth's Center (O) and measured to the satellite (A) and Sun (B). Note that this shadow function only takes into account the umbra and does not differentiate the penumbra from complete visibility of the Sun [2].

Higher fidelity can be achieved with the solar radiation pressure perturbation by more realistically modeling the dynamics of this pressure. Accurately modelling the cross-sectional area of the spacecraft instead of assuming a basic spherical or plate-like shape is one approach. Building on this, modelling the torques on the spacecraft body and how it dynamically effects the cross-sectional area exposed to the sun may greatly effect calculations, especially if those areas are shaped differently 
and have different material properties. Finally, if different material properties are being considered, there is a more complex expression that can be used to model the solar radiation pressure and its exerted force on a spacecraft. These equations consider the absorption, specular reflection, and diffuse reflection properties of the material covering the area exposed to the Sun [22].
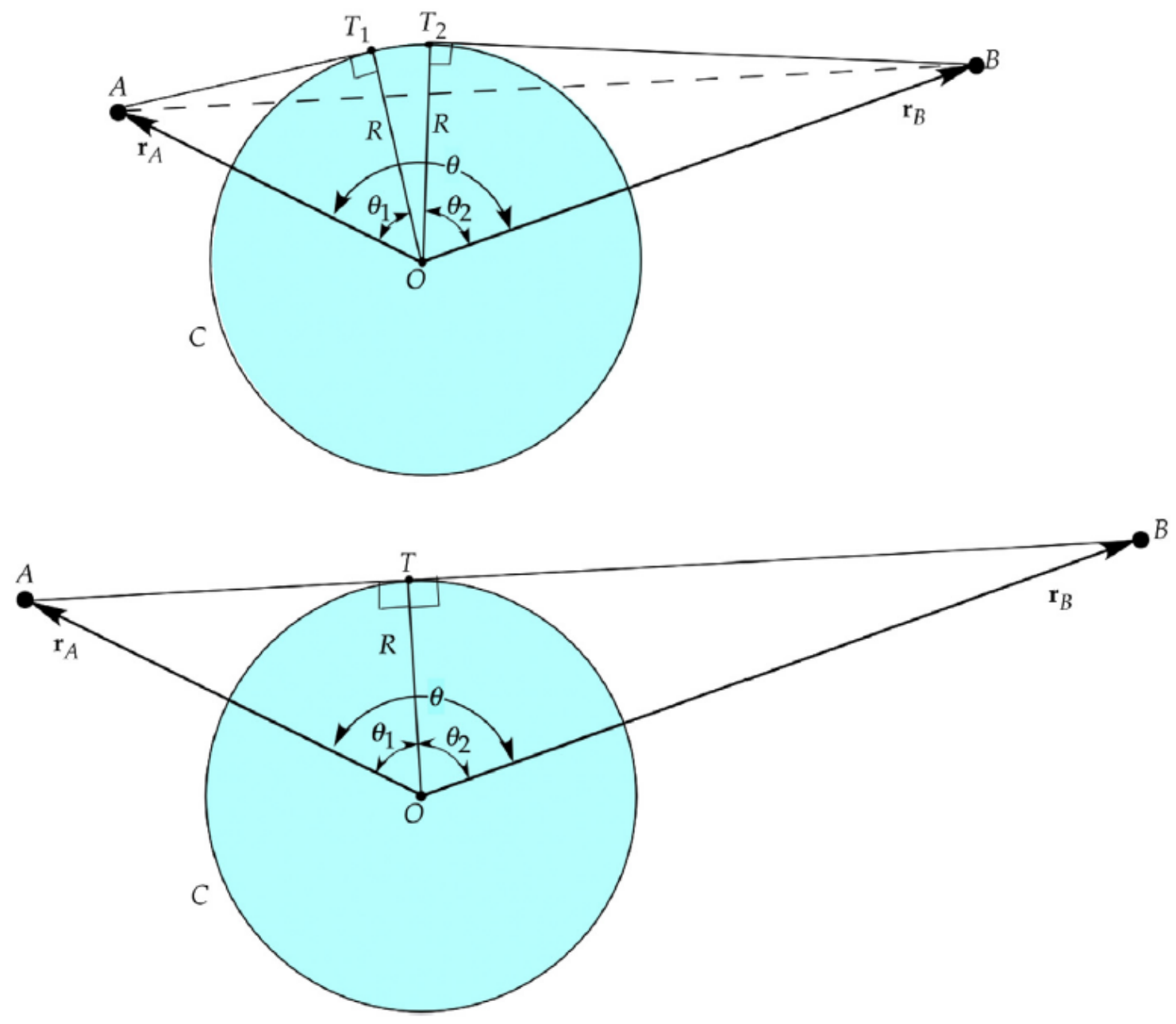

Figure 2.5: Shadow Function Angles [6]

\subsubsection{Excluded Perturbations}

A number of other perturbations were excluded from the propagator. Perhaps most well known is the effect of atmospheric drag. As altitude increases this effect decreases exponentially, becoming almost entirely negligible at the GEO regime. With 
regards to geopotential effects, initial analysis was conducted using only the $J_{2}$ and $J_{22}$ terms. Additional analysis was conducted using all geopotential terms up through $J_{33}$. The $J_{2}$ effect is the dominant geopotential effect for earth with acceleration effects on the order of $10^{-5} \mathrm{~m} / \mathrm{s}^{2}$. Typically, J3 would be the next strongest effect, but in the GEO regime the J22 effect is resonant and has a noticeable effect on these orbits. The tesseral effects of $J_{21}$ and $J_{31}$ allows analysis of certain behaviors in the geopotential wells, and the $J_{33}$ term has a resonant effect in GEO, though to a lesser extend than $J_{22}$. All other geopotential effects are cumulatively on the order of less than $10^{-7} \mathrm{~m} / \mathrm{s}^{2}$, so they are not included.

Less common perturbations include the earth's radiation pressure, earth tides, and relativistic effects. The earth radiation pressure is a non-conservative perturbation on the order of about $10^{-7} \mathrm{~m} / \mathrm{s}^{2}$. It is the caused by radiation being re-emitted by the Earth and exerting pressure on the spacecraft, similar to SRP. This is much more noticeable for LEO spacecraft, affecting these objects by $10-35 \%$ of the effect of SRP. Earth tides are a conservative force caused by the deformation of the Earth and its oceans due to the gravitational effects of the Sun and Moon. The acceleration effects for a spacecraft are on the order of $10^{-9} \mathrm{~m} / \mathrm{s}^{2}$ and again play a bigger role in LEO spacecraft. The largest tidal force is that of solid earth tides with accelerations proportional to $1 / r^{4}$. An order of magnitude smaller is the effect of ocean tides. Finally, for extremely high accuracy propagations, the likes of which require accuracy to $1 \mathrm{~cm}$, general relativistic effects can be included. For accuracy on the order of a tenth of a millimeter, relativistic effects from 3rd-body objects such as the Sun should be included.

\subsection{MATLAB Environmental Variables}

This propagator was developed and designed using MATLAB and its ode45 function to integrate the state of the object being propagated. To recreate the results of this paper, a number of MATLAB environmental variables are included for the reader in Table 2.5. The recommended Java Heap Memory is included as well; decreasing this may lead to MATLAB prematurely crashing due to memory errors. 


\section{Table 2.5: Environmental Variables}

\begin{tabular}{|c|c|}
\hline Setting & Value \\
\hline ODE Integrator & ode45 \\
\hline ode45 Relative Error & $10^{-8}$ \\
\hline ode45 Absolute Error & $10^{-8}$ \\
\hline Java Heap Memory & $512 \mathrm{MB}$ \\
\hline
\end{tabular}

\subsection{Using the Propagator}

The layout of the code is provided in Figure 2.6. Users begin by supplying relevant TLEs to the TLE parser which converts the TLE format to a set of classical orbital elements for each spacecraft. These are saved in a comma delimited file. The propagation code is then run with user input for the COEs of interest along with information regarding which propagator to use, which perturbations are included, whether to run the collision code, and whether to plot relevant orbit information. If the collider is being run, then the initial input will include the collider user settings as well: number of fragments, minimum generation size, maximum generation size, and satellite properties for mass and area for both the satellite and the theoretical object colliding with it. 

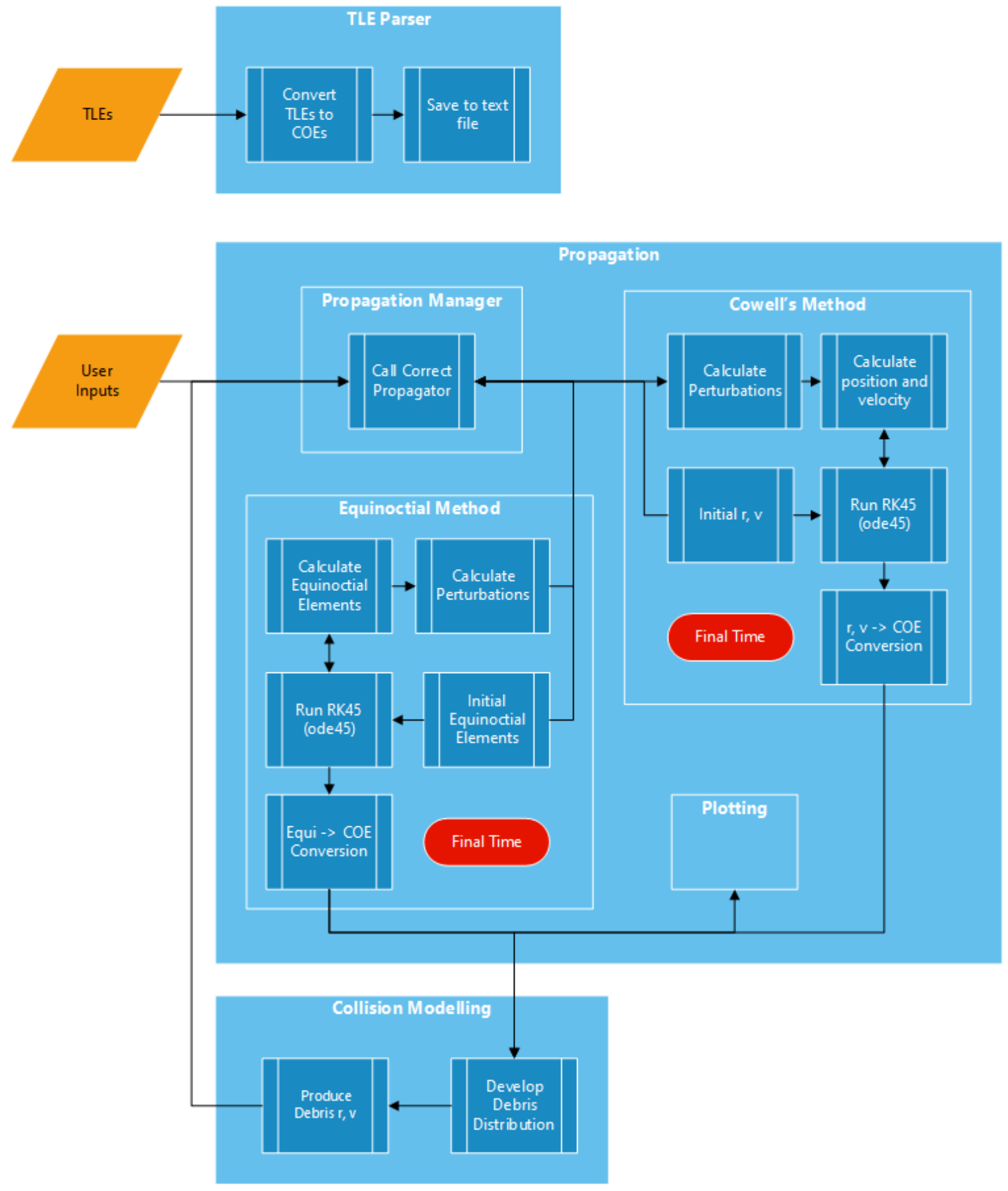

Figure 2.6: A layout of all elements of the code 


\section{Chapter 3}

\section{Collision Simulation}

In order to analyze the behavior of debris in the graveyard orbit, it is important to consider the breakups or collisions between the debris and the debris breakup model used to simulate them. All objects in this regime are uncontrolled and decommissioned, meaning corrective maneuvers cannot be enacted to avoid collisions. Additionally, if the recommended conditions of decommissioning is ignored in such a way that propellant tanks or electronics are at risk of exploding at a future time, decommissioned satellites can expect breakups even without coming into contact with a another piece of debris.

The collision modelling element of the code is presented in Figure 2.6 is used to model orbital breakup at GEO-altitude or higher and is designed to take into effect Hanada's experimental scaling of NASA's Standard Breakup Model for these altitudes [13].

\subsection{Previous Work}

The primary scope of collision breakup analysis has been done in the LEO regime. This is due to the higher number of satellite launches into this region of space, therefore a greater interest has existed. To analyze breakups in the LEO regime, the NASA Standard Breakup Model 2000 revision has been developed as just one type of fragmentation model. This model takes into account the area-to-mass distributions of two colliding objects, as well as their velocity vectors and mass values. This model is based on hypervelocity impact experiments done in a laboratory setting as well as 
study of the Solwind spacecraft on-orbit collision [13].

The NASA Standard Breakup Model cannot be directly applied to GEO-regime collisions without some modification. This is because the model is built on experimental data produced from hypervelocity impacts typical of LEO orbits, whereas GEO collisions are on the range of $800 \mathrm{~m} / \mathrm{s}$ or less. In collision cases between GEO and GEO Transfer Orbits (GTOs), this raises to only $1.5 \mathrm{~km} / \mathrm{s}$. These low-velocity impacts mean that GEO collisions can be considered non-catastrophic, and the underlying behavior of the satellite structures under impact differ from those in a LEO impact. A GEO revision to the NASA breakup model is provided by Hanada and is based on fitting the base model to experimental results simulating GEO collisions. This Hanada experimental scaling is used as the breakup model in this thesis.

\subsection{Method}

The collision simulator makes use of the Hanada breakup method. This method is defined by three distributions: a size distribution defining the characteristic length of each fragment, an area-to-mass distribution defining the ratio of each fragments area to its mass, and a $\Delta v$ distribution defining the difference in velocity from the colliding spacecrafts. Following the creation of these distributions, a Gaussian distribution is used to generate unit vectors for the $\Delta v$ distribution. This is then added to the velocity vector of the largest of the two objects in the collision to determine the velocity of each debris object resulting from the collision.

To begin analysis with the Hanada breakup method, the mass and states of two colliding spacecraft must be provided. An angle is measured between the two velocity vectors as

$$
\theta=\cos ^{-1}\left(\frac{\left.\vec{v}_{1} \cdot \vec{v}_{2}\right)}{\left\|\vec{v}_{1}||\right\| \vec{v}_{2} \|}\right)
$$

The collision velocity for a non-catastrophic impact can then be calculated:

$$
v_{c}=\sqrt{v_{1}^{2}+v_{2}^{2}-2 v_{1} v_{2} \cos (\theta)}
$$

The last calculation that must be made to setup the distributions is the defini- 
tion of the collision mass after non-catastrophic collision. The definition of a noncatastrophic collision is a collision with a ratio of kinetic energy at impact to target mass less than $40 \mathrm{~J} / \mathrm{g}$. A ratio below $40 \mathrm{~J} / \mathrm{g}$ characterizes a collision whether there is total fragmentation of the smaller object and cratering of the larger object. The non-catastrophic post-collision mass is defined as

$$
M=m_{s} v_{c}^{2}
$$

where $m_{s}$ is the mass of the smaller of the two objects. This differs from the definition of collision mass for a catastrophic impact typical of LEO collisions, which is included here for completeness and represents total fragmentation of both masses:

$$
M=m_{1}+m_{2}
$$

From here, the various distributions can be built. The size distribution is provided as

$$
N\left(L_{C}\right)=S * 0.1(M)^{0.75}\left(L_{C}\right)^{-1.71}
$$

where $N\left(L_{C}\right)$ is the normal distribution of the characteristic length $L_{C}$ of each fragment and $\mathrm{S}$ is a scaling factor $S=6$.

The next distribution is the area-to-mass distribution. First, most fragments are assumed to be plate-shaped so that the average cross-sectional area of the fragments formed by collision can be calculated as

$$
A_{s}=\frac{1}{2}\left(L_{C}^{2}+2 L_{C} z\right)
$$

where $z$ is the thickness of the plate. Based on the plate assumption, the mass can be calculated as

$$
M=\rho z L_{C}^{2}
$$

where $\rho$ denotes the density of the material being used. An example of an acceptable 
density is given by the NASA Standard Breakup Model as

$$
\rho=92.937 L_{C}^{-0.74}
$$

The area-to-mass ratio is then evaluated as

$$
\frac{A}{M}=\frac{\frac{L_{C}}{z}+2}{2 \rho L_{C}} .
$$

It is important to note that a lower boundary exists on the ratio based on the definition of characteristic length, which states that $L_{C} / z \geqslant 1$ should be satisfied. The lower bound is given as

$$
\frac{A}{M} \geqslant \frac{1.5}{\rho L_{C}}
$$

Hanada concludes that, with the use of this lower bound, the area-to-mass distribution model developed by NASA for its standard breakup model is still relevant and can be applied for GEO collisions. The distribution is defined as

$$
D_{A / M}^{S O C}\left(\lambda_{C}, \chi\right)=N\left[\chi ; \mu^{S O C}\left(\lambda_{C}\right), \sigma^{S O C}\left(\lambda_{C}\right)\right]
$$

where

$$
\lambda_{C}=\log _{10}\left(L_{C}\right), \quad \chi=\log _{10}\left(\frac{A}{M}\right)
$$

and $N$ is a normal distribution in $\chi$ about a mean value that differs with the fragments characteristic length,

$$
\mu^{S O C}\left(\lambda_{C}\right)= \begin{cases}-0.3, & \lambda_{C} \leqslant-1.75 \\ -0.3-1.4\left(\lambda_{C}+1.75\right), & -1.75 \leqslant \lambda_{C}<-1.25 \\ -1.0, & -1.75 \leqslant \lambda_{C}<-1.25\end{cases}
$$

with a standard deviation of

$$
\sigma^{S O C}\left(\lambda_{C}\right)= \begin{cases}0.2, & \lambda_{C} \leqslant-3.5 \\ 0.2+0.1333\left(\lambda_{C}+3.5\right), & \lambda_{C}>-3.5\end{cases}
$$


As for the $\Delta v$ distribution, Hanada offers two solutions: an upper bound can be placed on the Standard Breakup Model equations as

$$
\Delta v \leqslant 1.3 v_{c}
$$

or a normal distribution about $\chi$ can be used where

$$
\mu=0.45 \chi+1.45, \quad \sigma=0.27
$$

At this point, the $\Delta v$ can be applied to generate the states of the fragments at the location of collision. The velocity of each fragment is calculated as

$$
\vec{v}=\vec{v}_{1}+\left(\Delta v * D C M(\text { rand }) * \hat{v}_{1}\right)
$$

where $v_{1}$ is the velocity of the largest object and $D C M($ rand $)$ is a rotation matrix generated from three rotation angles created using a Gaussian distribution. This means that the velocity of each fragment is some deviation from the largest parent object's state, the deviation of which is determined by a random direction of magnitude $\Delta v$.

\subsection{Using the Collision Code}

The collision code can be started either within the orbital propagator code or externally on a set of inputs. Inputs to the collision code are the state vectors of two colliding spacecraft and their respective masses, as well as an optional parameter for the number of fragments that should be generated. Fragmentation is assumed to be at GEO altitude for non-catastrophic collision debris generation. After execution of the code, a list of state vectors for the debris is generated, along with each of the distributions. This debris can then be propagated using the propagator. Doing so allows for an understanding of the behavior of the debris cloud in the GEO disposal regime. 


\section{Chapter 4}

\section{Results}

The code developed to study the debris environment has gone through two different sets of validation and result examination: one for the propagator and the second for the collision breakup model. Following these two validations, results were generated and consisted of the propagation of debris resulting from a collision. These results consist of an analysis of the longitudinal behavior of a collisions fastest and slowest fragments to see how the behavior of the fragments differ from their parent objects.

\subsection{Propagator Validation}

The propagator validation phase consists of plotted comparisons between classical orbital elements, longitude and latitude generated by this code and an existing validated propagator: STK 10. A set of classical orbital elements are produced for each object being used for validation and the elements are then converted into state vectors. The state vectors act as input into the propagators, which will calculate the sum perturbative acceleration at each point in time and add it to the gravitational acceleration acting on the spacecraft. The outcome is a matrix of classical orbital elements for each object, which can then be plotted for analysis and comparison.

For each satellite considered in the validation phase, the satellite number, name, estimated $\mathrm{A} / \mathrm{m}$ ratio, and additional nodes are provided in Table 4.1. This selection of satellites includes at least one representative piece of debris for each classification of interaction with the geopotential wells of the graveyard regime. Where $\mathrm{A} / \mathrm{m}$ estimates 
are not provided, the $\mathrm{A} / \mathrm{m}$ is determined using Equation 1.1 with a $C_{R}$ of 2 . If the propagation of these chosen debris objects can be accurately modelled, then the propagator can be validated for GEO propagation and the simulation of each type of geopotential well's effect on GEO spacecraft. Accurate modelling is determined based on the plotted trends of the results and how close they match to STK output, TLE trends, and additional knowledge of the satellite or regime.

Table 4.1: Set of Satellites Used For Testing

\begin{tabular}{|c|c|c|l|}
\hline Satellite Number & Name & A/m (Est.) & Notes \\
\hline $\mathbf{0 3 4 3 2}$ & Titan 3C Transtage R/B & - & $\begin{array}{l}\text { Launched 1968-09-26. } \\
\text { Drifter. }\end{array}$ \\
\hline $\mathbf{0 4 0 6 8}$ & ATS 5 & 0.00988 & $\begin{array}{l}\text { Launched 1969-08-12. } \\
\text { Drifter. }\end{array}$ \\
\hline $\mathbf{0 4 2 5 0}$ & Skynet 1 & $\begin{array}{l}\text { Launched 1969-11-22. } \\
\text { Trapped in W Well. }\end{array}$ \\
\hline $\mathbf{0 5 5 8 8}$ & OPS 9432 & 0.0128 & $\begin{array}{l}\text { Launched 1971-11-03. } \\
\text { E-W Trapped. }\end{array}$ \\
\hline $\mathbf{2 9 6 4 0}$ & Fengyun 2D & 0.0714 & $\begin{array}{l}\text { Launched 2006-12-08. } \\
\text { Trapped in 75E Well. }\end{array}$ \\
\hline
\end{tabular}

Six different plots are considered in the validation, these show the trends of each object over time for: angular momentum, semi-major axis, eccentricity, right ascension of the ascending node, argument of perigee, inclination, longitude, and latitude. The first five of these describe the orbit of the satellite, and the longitude and latitude help to relate the satellites position to that orbit. The latitude and longitude are looked at opposed to the true anomaly because trends in both can be observed to determine if the propagator is producing the results that signal an accurate propagation. For example, all latitude are expected to remain below 15 degrees, and for a West trapped object the longitude would be trapped in the [-180,0] degree range.

Each set of plots will compare trends from multiple sources. The plots compare the following: STK/Equinoctial/Cowell, TLE/STK/Cowell, and STK/Equinoctial(No SRP)/Cowell. Plots are produced both for a short-term, 10 day analysis and for a longer period. This longer period varies for each satellite and is determined by the range of historical data the TLEs supply for each object across its decommissioned phase of life. TLE in this case represents TLE data retrieved from Celestrak. STK 
is data generated by STK 10 and the HPOP propagator with the following perturbations: 32x32 gravity model using the WGS84_EGM96 model, solar radiation pressure for a spherical object and a dual cone shadow model using the true Sun position, Earth and Moon as eclipsing bodies, 3rd body gravitational forces of the Sun, Moon, and Jupiter, Full Earth solid tides including time-dependent terms, 4x4 ocean tides, albedo and thermal Earth radiation pressure forces where $\mathrm{Ck}$ is 1.0, and relativistic accelerations. The HPOP propagator uses a RKF 7(8) integrator with variation of parameters of order 7. A relative error is set at $1.00 \mathrm{e}-008$. The reason for the use of such a complex set of perturbations in the STK plots is to show the capabilities of the other simpler propagators versus a commercial, validated propagator AGIWebsite. One assumption being tested in this thesis is that the perturbations modeled in the equinoctial and Cowell propagators is comprehensive enough to accurately model the behavior of GEO debris. By identifying a simple set of perturbations that accurately model GEO wells, time can be saved in future propagations for objects near wells and in the graveyard orbit.

Equinoctial plots utilize $J_{2},{ }_{J 22}$, solar, lunar and SRP effects and account for Earth as an eclipsing body, and use the variation of parameters method with a modified equinoctial element propagator produced for this paper. The Equinoctial (No SRP) trends will use the equinoctial propagator with all of these perturbations except the SRP. The Cowell plots utilize the same forces as the equinoctial propagator but using a Cowell's method approach. Later on in this validation, a variant set of perturbations will be looked at for the Cowell propagator that includes geopotential terms up to $J_{33}$. Both equinoctial and Cowell methods use an RKF45 integrator with a relative and absolute error of 1.00e-008.

\subsubsection{Comparison Plots - STK and Custom Propagators}

These plots compare the trends for each object over a short-term and a long-term time span between STK, the Equinoctial propagator, and the Cowell propagator. The STK propagator has been validated by external sources, so a valid propagator should be capable of matching trends to STK provided they use the same perturbations. A sophisticated set of memory and computationally expensive perturbations are used 
in STK and compared against a more limited set of elements in the Equinoctial and Cowell propagators that should closely match. In plots where the Equinoctial line is not evident, this is because the Cowell plot matches exactly with the Equinoctial output. Both Cowell and Equinoctial propagators utilize the same perturbations until otherwise stated.

The first plot is provided in Figure 4.1 and is short-term, 10 day propagation data for satellite 03432 . In each of these subplots, the equinoctial propagator matches the output of the Cowell propagator. Slight variations can be observed in the angular momentum, semi-major axis, inclination, and RAAN when comparing the custom propagators to the STK output. The longitude and latitude are near-perfect matches to STK, but the argument of perigee and eccentricity require discussion. The eccentricity is a minor difference at this point, but this signals a much larger oscillation on the long-term form of this plot. The large variation in argument of perigee shows noticeable inaccuracy, but this again has new meaning in the long-term plots. As the propagation of this satellite progresses, this error decreases and continues to oscillate around the trend of the STK calculation.

As the long-term form of Figure 4.1, Figure 4.2 show similar performance between the custom propagators and the STK output. Checking the longitude plot shows confirmation that this is a drifter object and the longitudinal behavior was accurately captured. Likewise, the latitude matches STK and expected behavior of a GEO spacecraft's latitude range. The only trend that shows disparity between the Cowell and Equinoctial propagator is the semi-major axis, showing that in fact the equinoctial propagator is farther from the STK baseline than the Cowell output. This is unexpected if both Cowell and Equinoctial propagators are acting the same, as the Equinoctial propagator should minimize the inaccuracies that accrue at each time step. Of final note, the eccentricity of the custom propagators oscillates near the STK baseline but varies by a large degree, and this only increases as time goes on. For propagations extending over the 16-year period shown here, it is recommended that additional perturbations be included in analysis. 

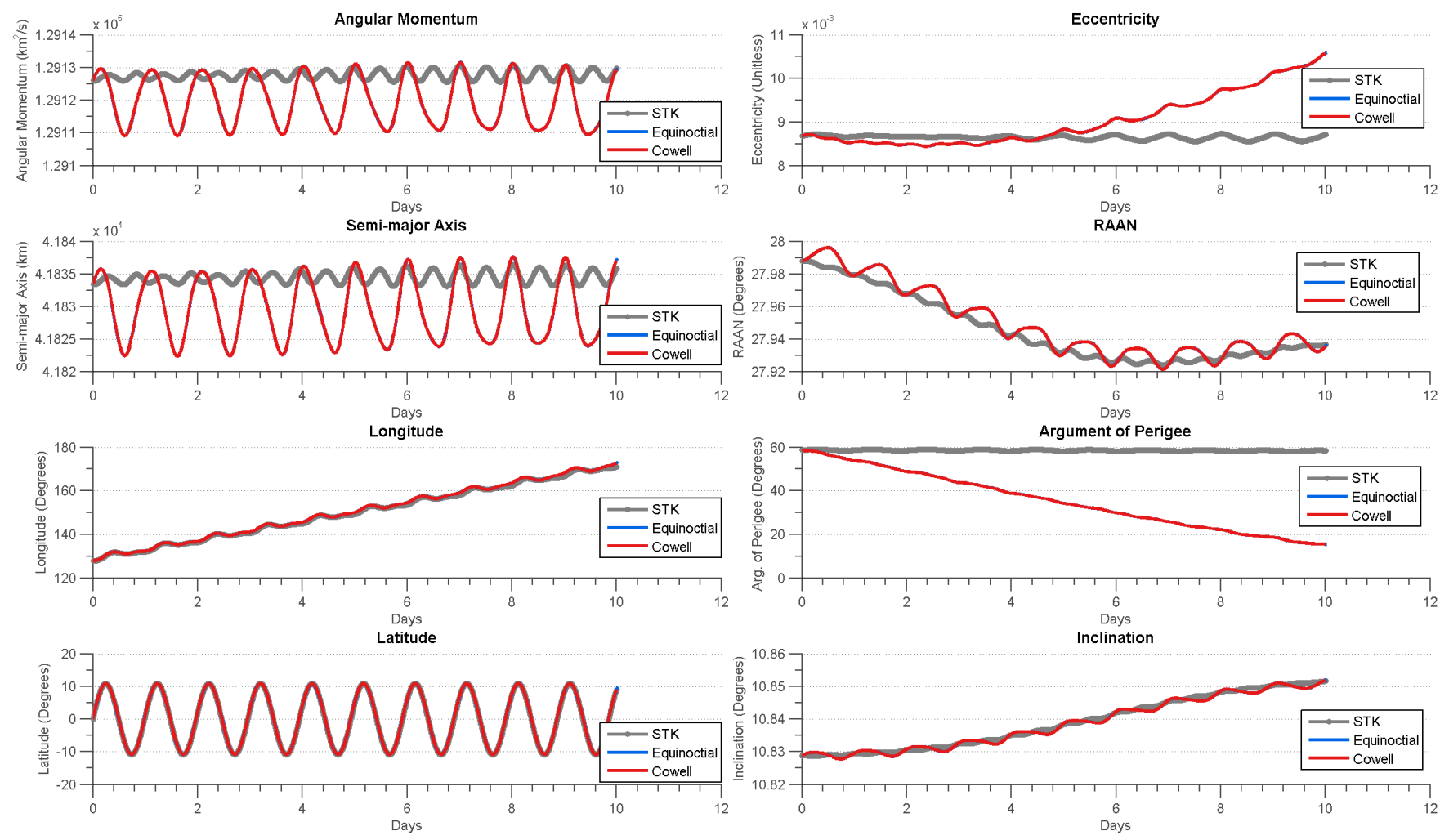

Figure 4.1: Short Term Versus Plots for Satellite 03432
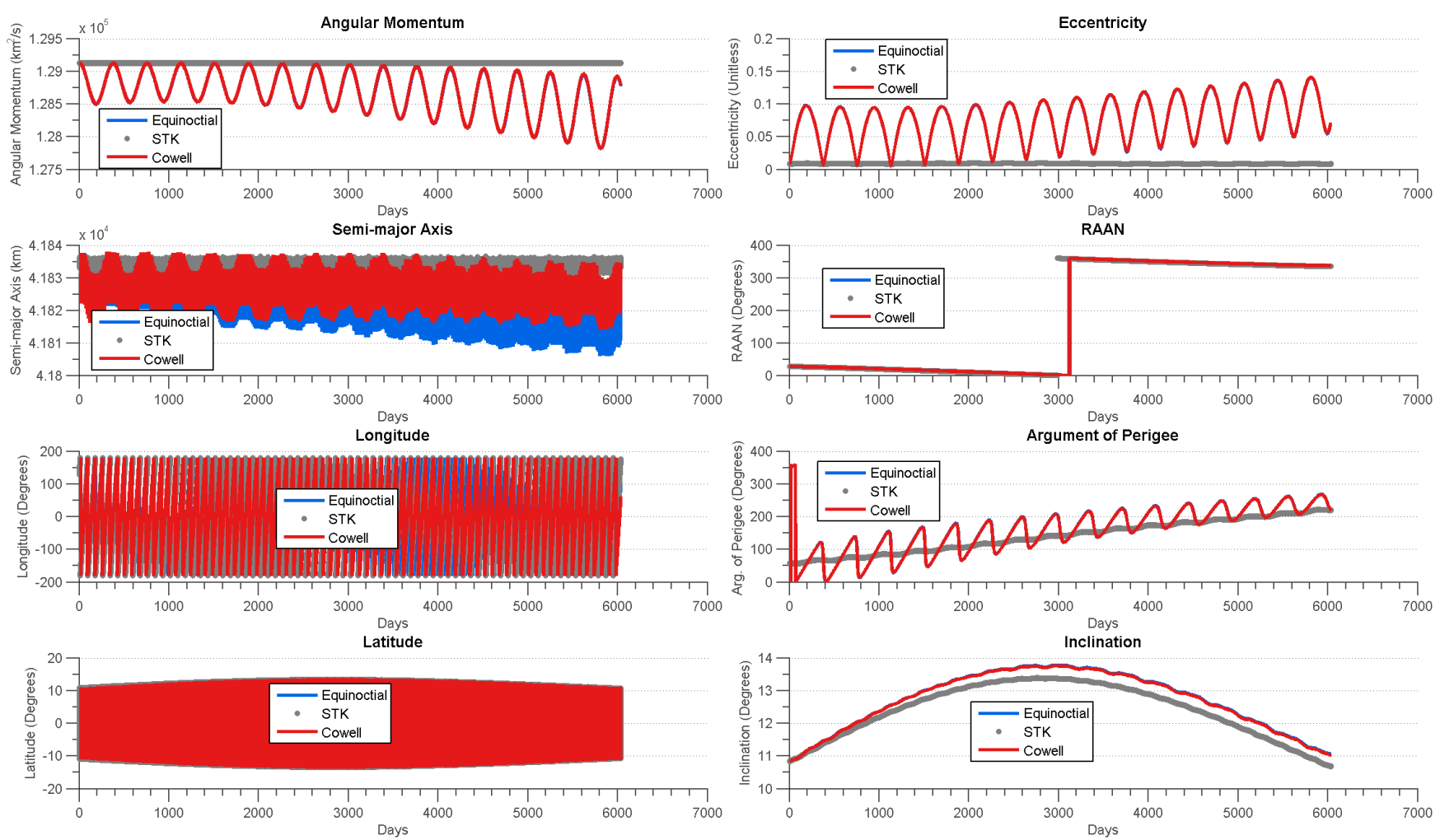

Figure 4.2: Long Term Versus Plots for Satellite 03432 
The short-term plots for satellite 04068, ATS 5, is shown in Figure 4.3. This satellite is also a drifter, the behavior of which isn't clear on this short time span, but there is a noticeable difference in the longitude. The other plots show similar behavior as witnessed in satellite 03432, where Cowell closely mimics the behavior of STK. It is also obvious that the Equinoctial results have a much larger variation from STK here, which is once again unexpected. The long-term plot is shown in Figure 4.4 and contains additional information.
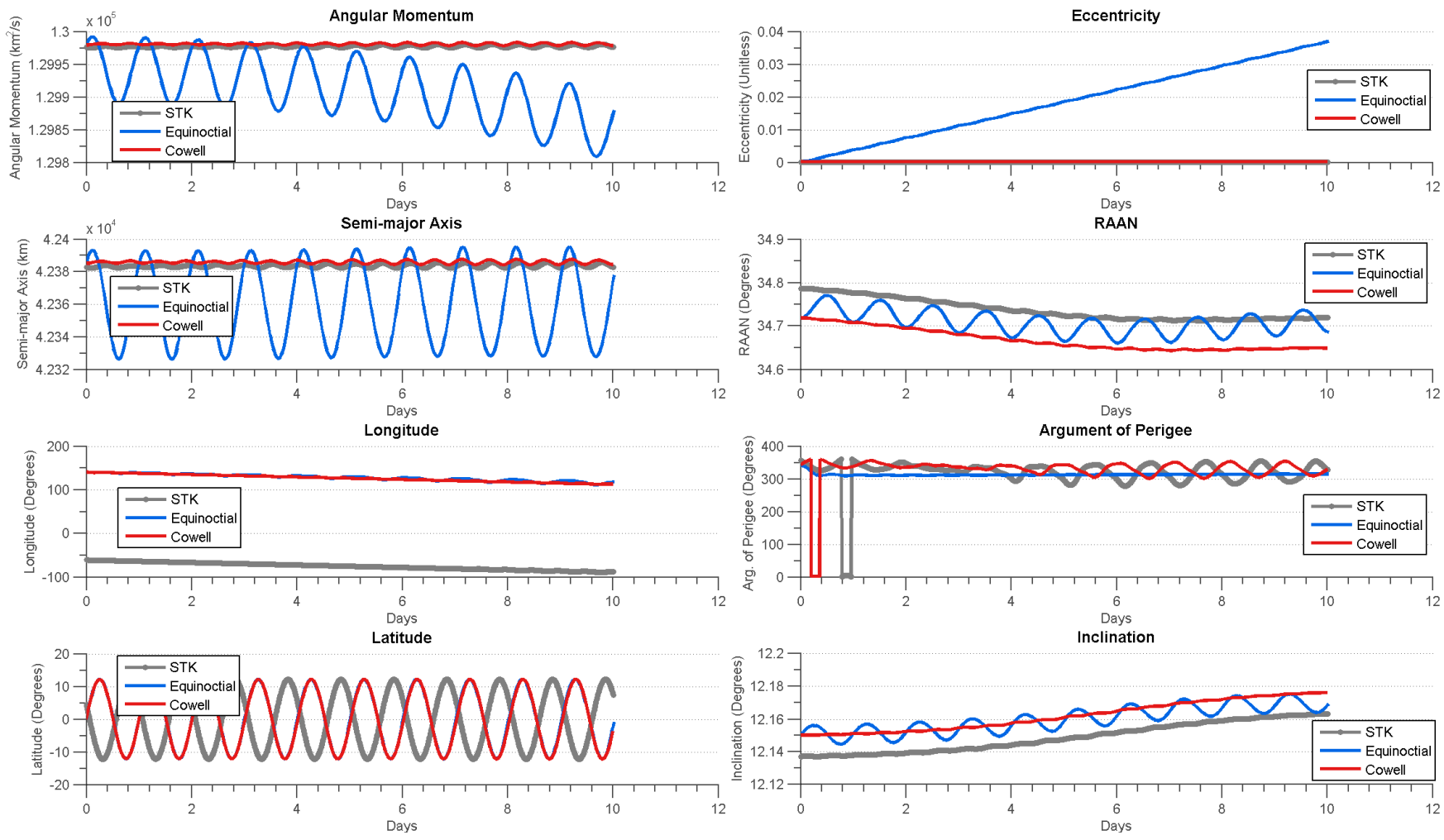

Figure 4.3: Short Term Versus Plots for Satellite 04068

The most important concept to understand from this plot is that the Cowell method has a much easier time matching the STK output. Both Equinoctial and Cowell methods have a fairly accurate match to the longitude and latitude behavior of the object in question, but the COEs of the Equinoctial plots begin to vary wildly, showing that the Equinoctial propagator may have unaddressed issues. 

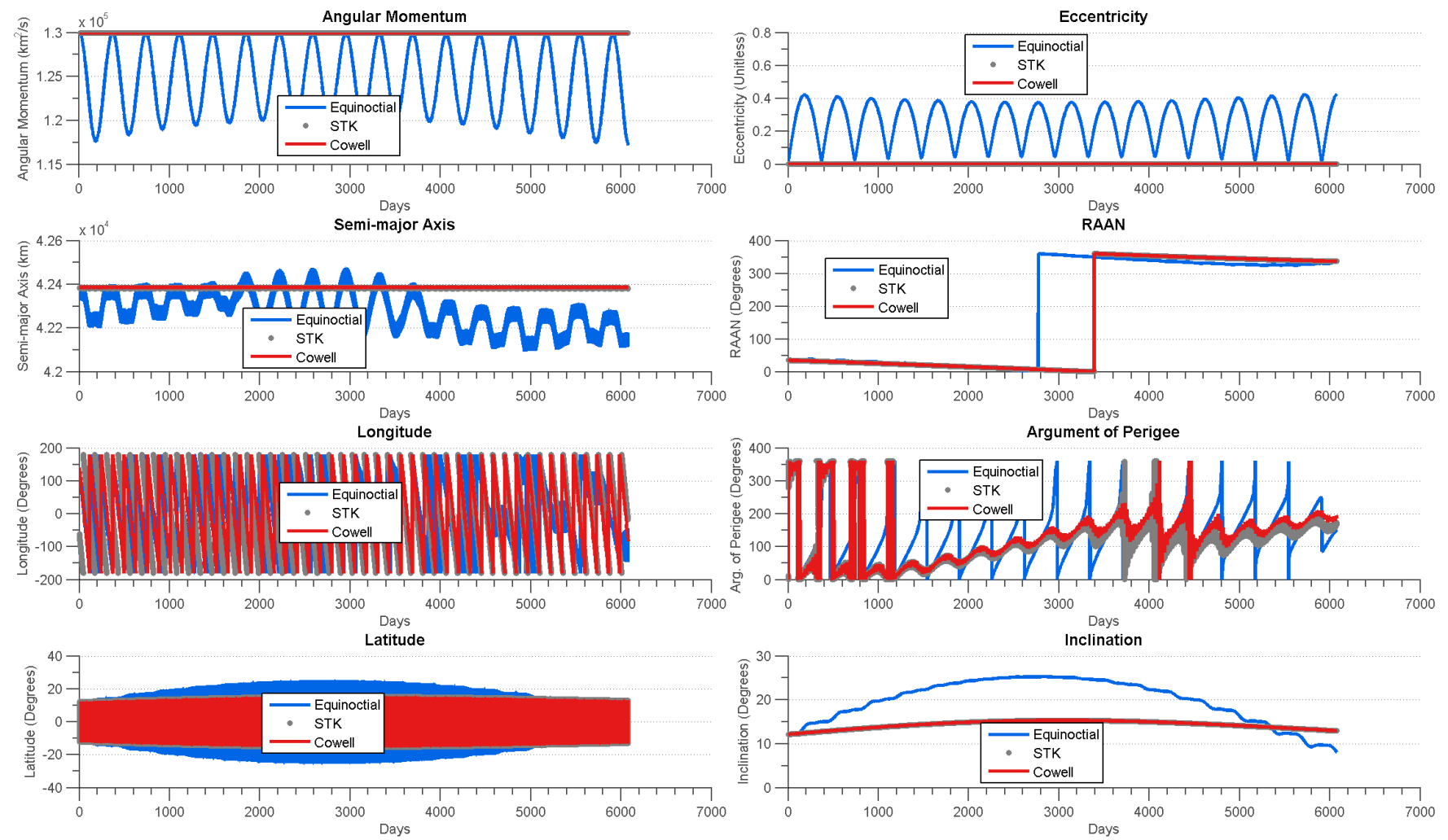

Figure 4.4: Long Term Versus Plots for Satellite 04068

The comparisons continue with the short-term and long-term plots of satellite 04250 in Figure 4.5 and Figure 4.6, respectively. In the short-term, the custom propagators have the same performance and the only noticeable issue at this level is that the eccentricity grows from the STK baseline and the argument of perigee steadily drifts away. On the long-term range, there is clearly an issue in both custom propagators at the beginning, but the Cowell propagator eventually slides into the expected behavior of the West Well trapped object. The Equinoctial propagator fails to recover however, entering a drifter state. It is clear that the Equinoctial propagator is failing to accurately model the behavior of these satellites, a trend that will continue with the other validation cases. 

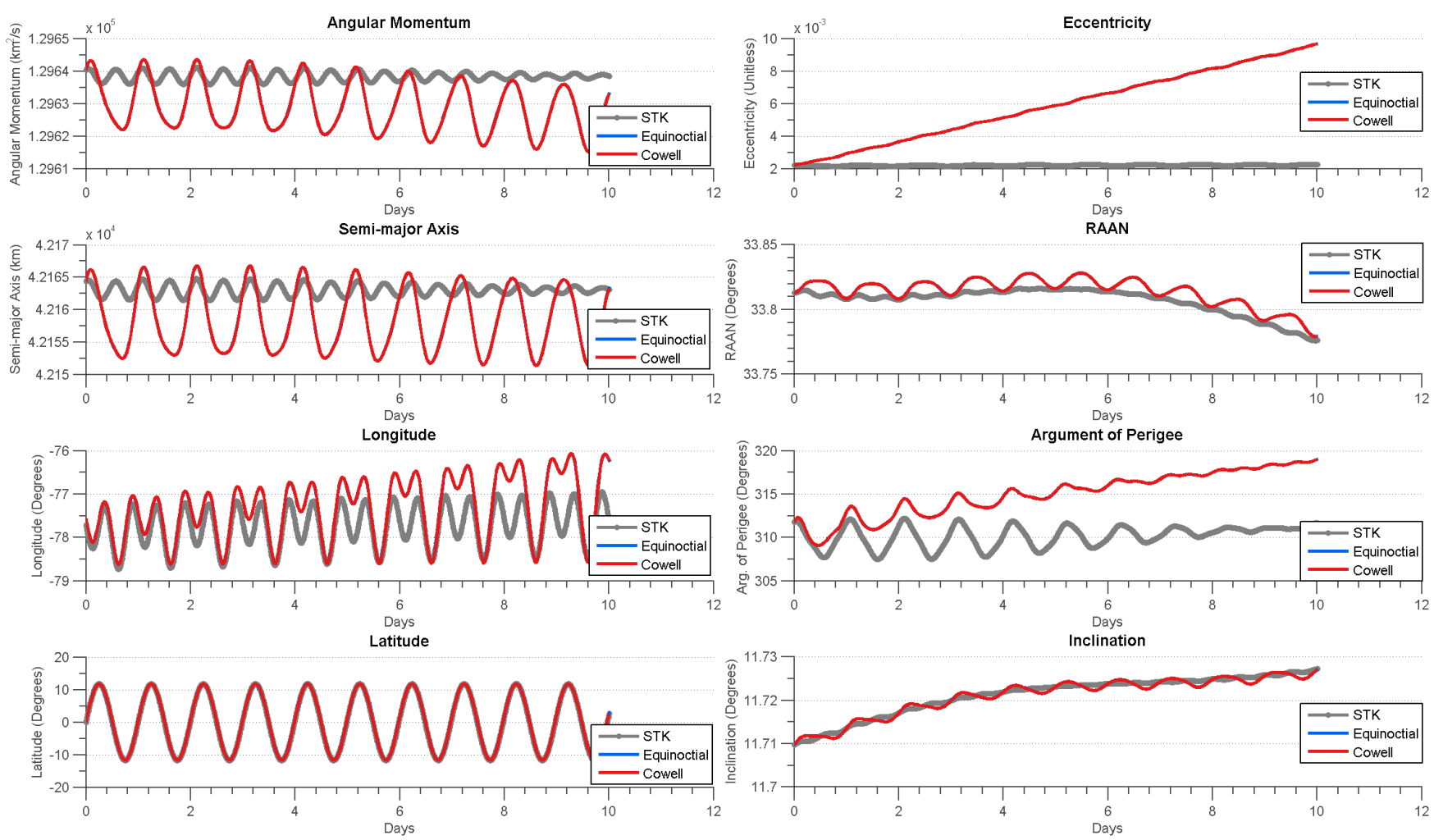

Figure 4.5: Short Term Versus Plots for Satellite 04250
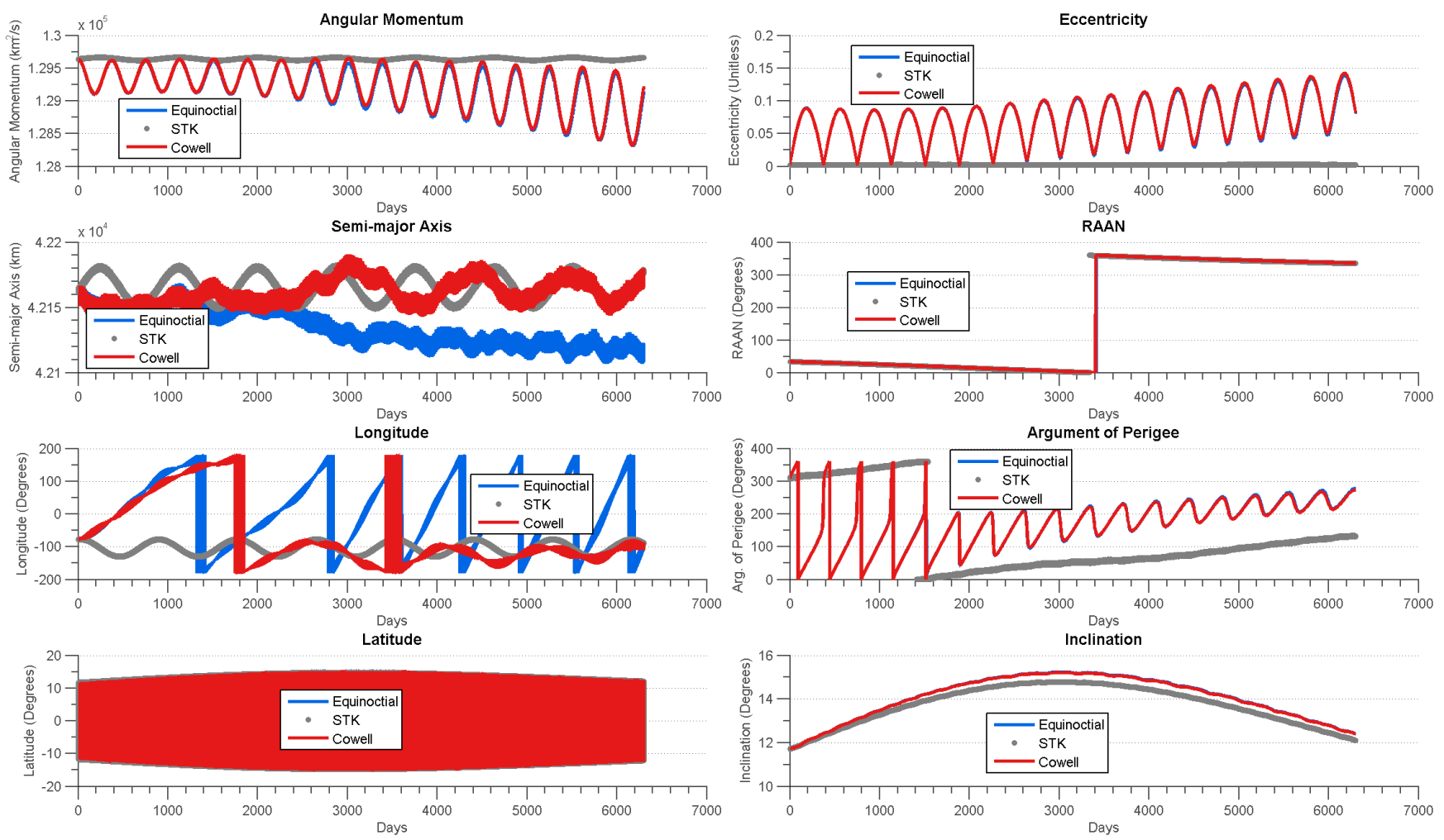

Figure 4.6: Long Term Versus Plots for Satellite 04250 
The validation continues with the short and long-term plots of satellite 05588 in Figure 4.7 and Figure 4.8. Both custom propagators fail to match the STK baseline in the short-term, and this issue continues in the long-term. This object is identified as an E-W trapped object according the ESA DISCOS database - all custom propagators however, identify this object as a drifter. Discussion on this issue will be continued in upcoming plots comparing the custom, STK, and TLE propagations, but these plots clearly identify a failure on the part of both custom approachs in providing a solution to the modelling of E-W well entrapment.
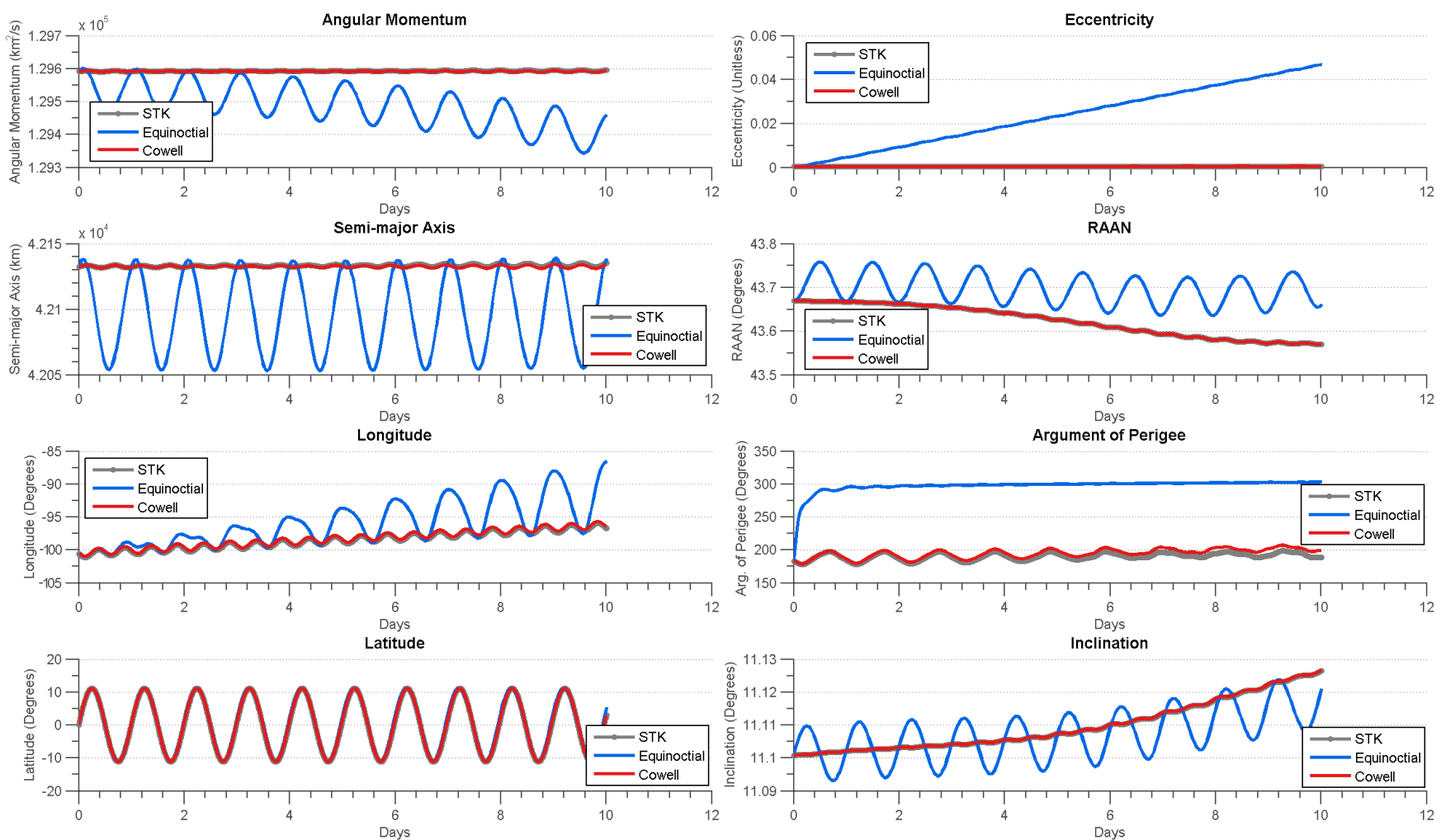

Figure 4.7: Short Term Versus Plots for Satellite 05588 

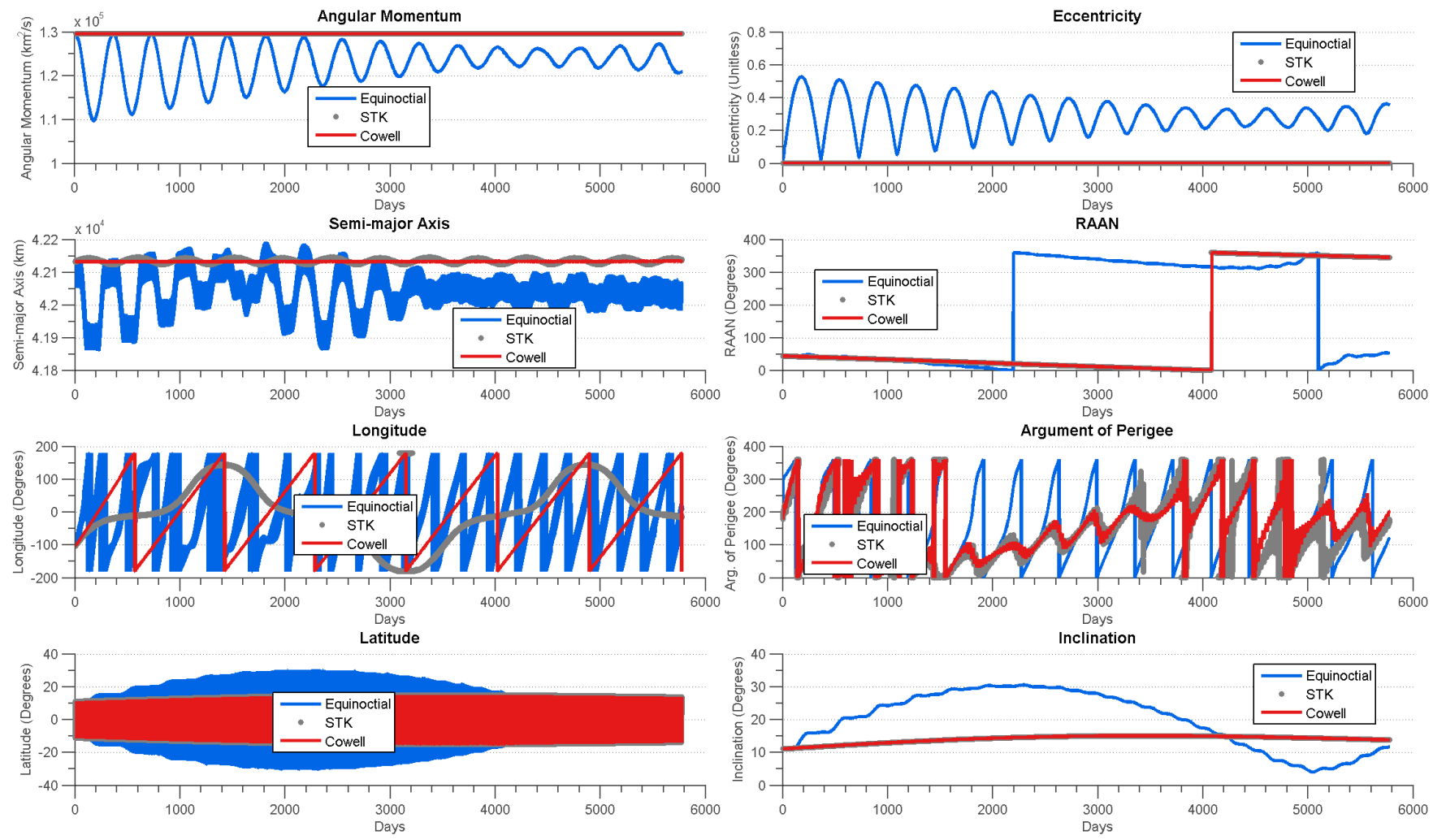

Figure 4.8: Long Term Versus Plots for Satellite 05588

The last set of versus plots are for satellite 29640 in Figure 4.9 and Figure 4.10. The Equinoctial propagator once again fails the capture the behavior established in the STK output, both on the long and short-term. This extends to longitude behavior, which Cowell can successfully capture, albeit at a lower accuracy to STK. Due to the inability of the Equinoctial propagator in matching the expected behavior of the satellites, this thesis will opt to use the Cowell propagator to continue analysis.

The second important finding from this analysis is that the Cowell propagation scheme is satisfactory for capturing all but EW-well entrapment. Drifting, East well, and West well behavior has been validated for the Cowell propagator and is capable of being modelled with the small set of perturbations selected for this method. It has been shown that this limited set of perturbations was not enough to model the EW-well entrapment, however STK has managed to do so. Though $J_{2}$ and $J_{22}$ alone cannot cover this behavior, it was believed that the tesserals $J_{21}$ and $J_{31}$ along with sectorial $J_{33}$ will provide the missing perturbatory effects to accurately simulate this behavior [1], [20]. Because of the success of the Cowell propagator in the other cases, 

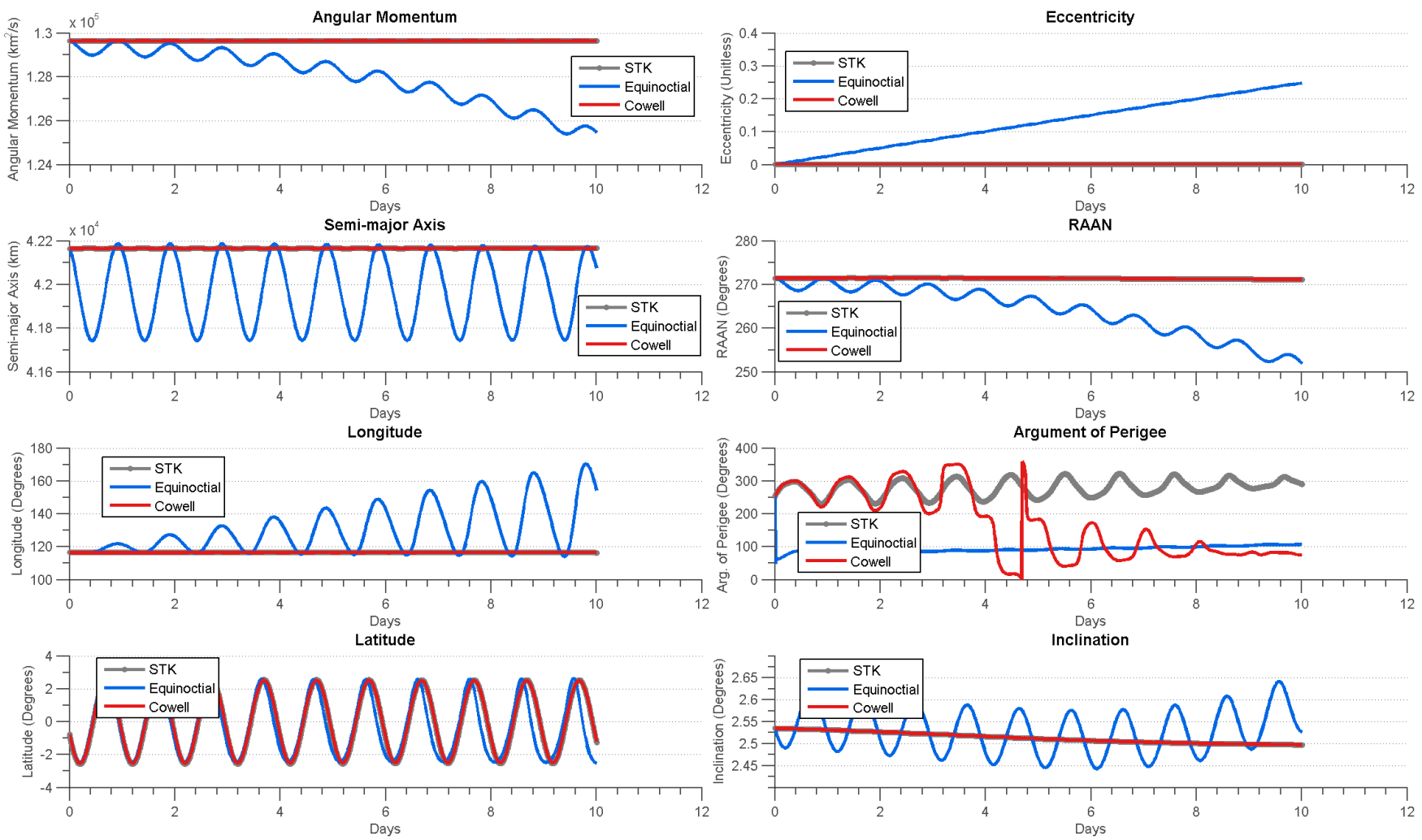

Figure 4.9: Short Term Versus Plots for Satellite 29640

it was chosen as the custom propagator that will be used for results analysis. First however, the propagator is upgraded from $J_{2}$ and $J_{22}$ to a $3 \times 3$ geopotential. All other perturbations are maintained. 

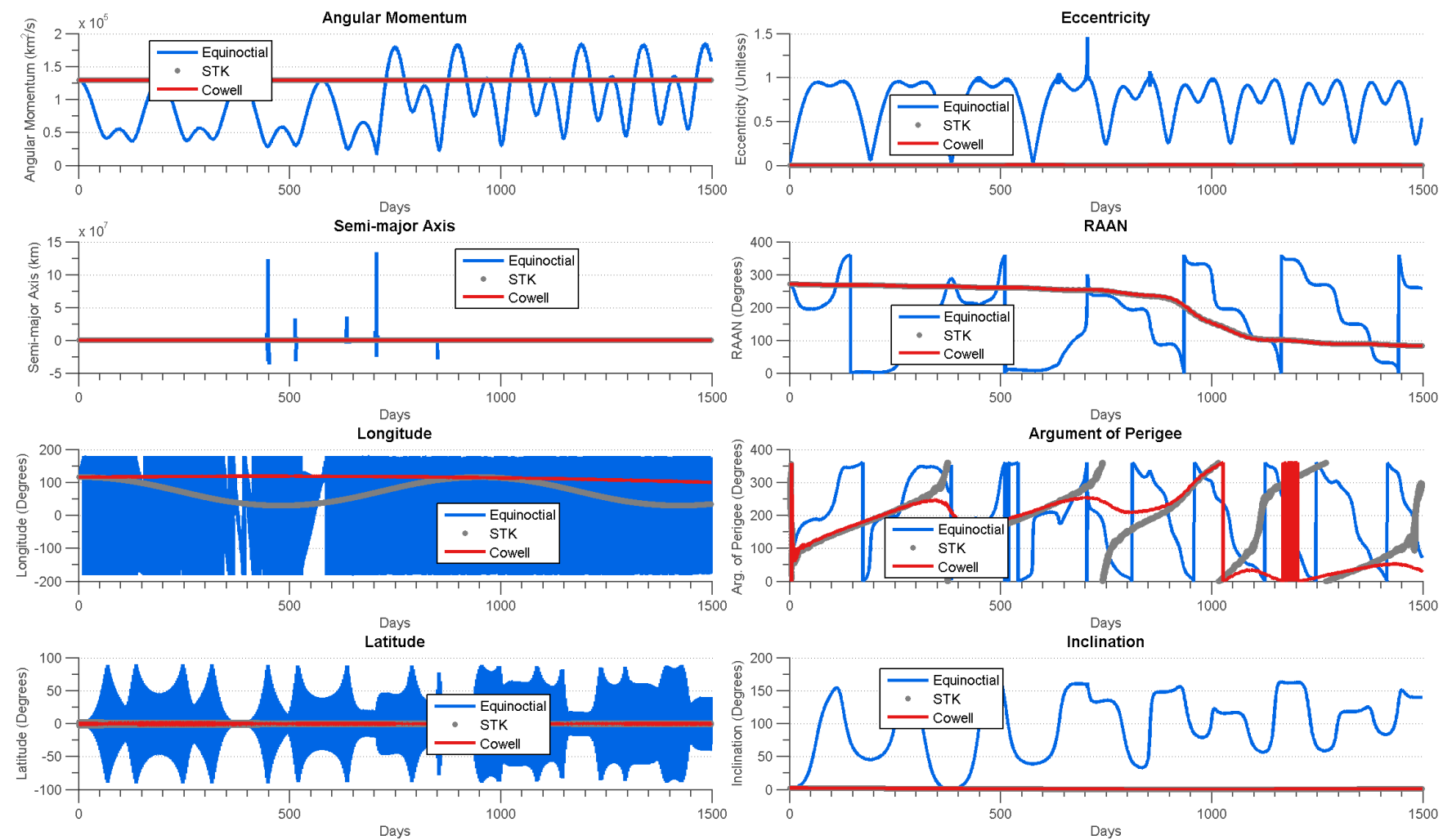

Figure 4.10: Long Term Versus Plots for Satellite 29640

With the new Cowell propagator, the long term plot of satellite 05588 is revisited. This is shown in Figure 4.11. While there is indeed a slight diversion of Cowell results from the STK baseline, the expected EW-well entrapment trend is matched. What appears as a block of red in the longitude plot is actually a graphical artifact due to the longitude sitting close to -180 degrees. When the value slightly decreases below -180 degrees, the bounding of the angle between $[-180,180]$ causes the value to appear at the maximum end of the bound. Then when longitude slightly increases from this value, it returns to the bottom of the bound again. This repeats over a period of 200 days before the variation is large enough that this is no longer a problem. This is additionally a good sign that the propagator is behaving as expected, because we see a similar effect in the STK plot. Notice the small number of points at the top of the STK trend even though the bottom of the trend is maintained. This is because the STK trend is being plotted with bullets instead of a line like the Cowell propagators. The other cases have been revisited with this upgraded Cowell propagator and results can be found in Appendix A. In non-drifting cases, the revised Cowell propagator matches 
STK longitudinal trends much better, and additional improvements are gained in some of the COE trends. In drifting cases, performance is equivalent. At this point, the Cowell propagator utilizing the $3 \times 3$ geopotential is verified for all geopotential well cases and capable of conducting results analysis.
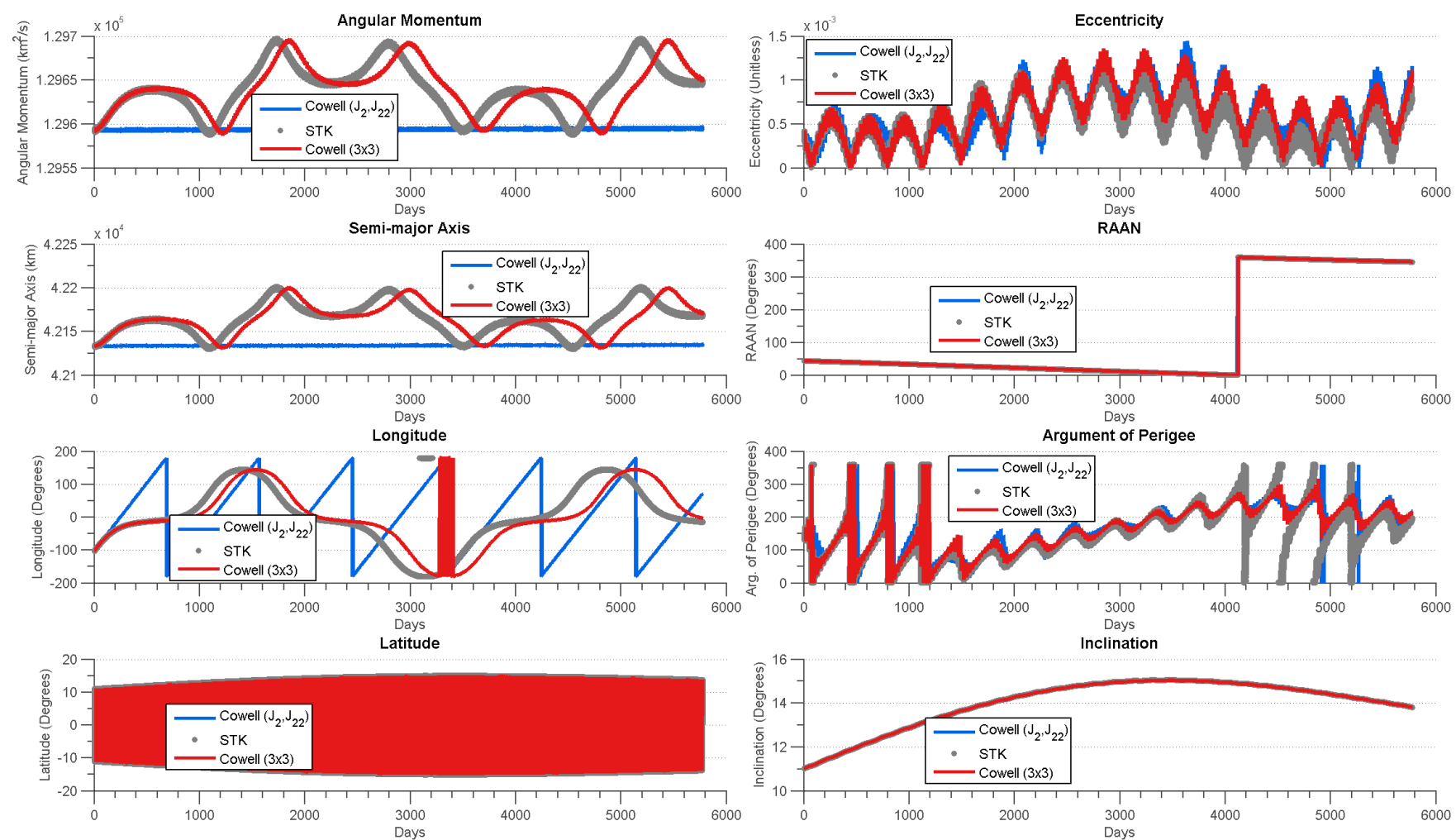

Figure 4.11: Long Term Versus Plots for Satellite 05588 with 3x3 Geopotential for Cowell

\subsubsection{Comparison Plots - No-SRP Equinoctial Propagator}

Before moving on, an explanation must be supplied for why the equinoctial propagator fails to match the expected outcome. The original assumption of this thesis was that the equinoctial propagator would greatly exceed the accuracy that the Cowell method was capable of meeting. As the code was developed for the equinoctial propagators, perturbations were first tested for accuracy one at a time using test cases presented in Curtis [6]. The equinoctial propagator succeeded in matching sample output for each test case except for the SRP, where the angular momentum trends slowly diverge over time as shown in Figure 4.12. The Cowell line of this plot repre- 
sents the expected value and shows that the same perturbation equations applied to Cowell's method do not have an issue matching the desired result.

The single difference between how the SRP perturbation is applied between both methods is that a rotation from the ECI frame into the RSW frame is applied during the Equinoctial propagator. This is a requirement as all perturbations must be broken into RSW components in order to be added into the Equinoctial elements during numeric integration. Multiple approaches to this frame rotation were taken (see Curtis [6] and Vallado [35]) and each approach was tested on example cases first to validate them, but changing the approach to the frame rotation did not correct the issue. Additionally, this frame rotation is used in the other perturbations without issue. With the Equinoctial propagator accurately simulating all other perturbations, it seems unlikely that the variation of parameter equations identified for the Equinoctial propagator are incorrect. However, of four different references for the Equinoctial VoP equations, only one pair of these papers had exactly matching equations, and they shared references to one another [14], [28], [16], [31]. Future work with the Equinoctial parameter set should revisit their derivation, as additional sources on their use is scarce. The variation in the angular momentum is only about $0.02 \frac{\mathrm{km}^{2} / \mathrm{s}}{\mathrm{day}}$, but over the multi-year analysis needed to confirm accurate GEO behavior, the results are unusable in the Equinoctial case. This error is also not a fixed inaccuracy per time step for all cases. Instead, it varies for each item of debris being propagated.

For comprehensive coverage of this issue, the variation plots showing the performance of the equinoctial propagator without the SRP perturbation is included in Appendix B. This is provided to show just how capable the propagator is of covering these variations without accounting for solar radiation pressure, but SRP is still necessary to accurately model the GEO case. 


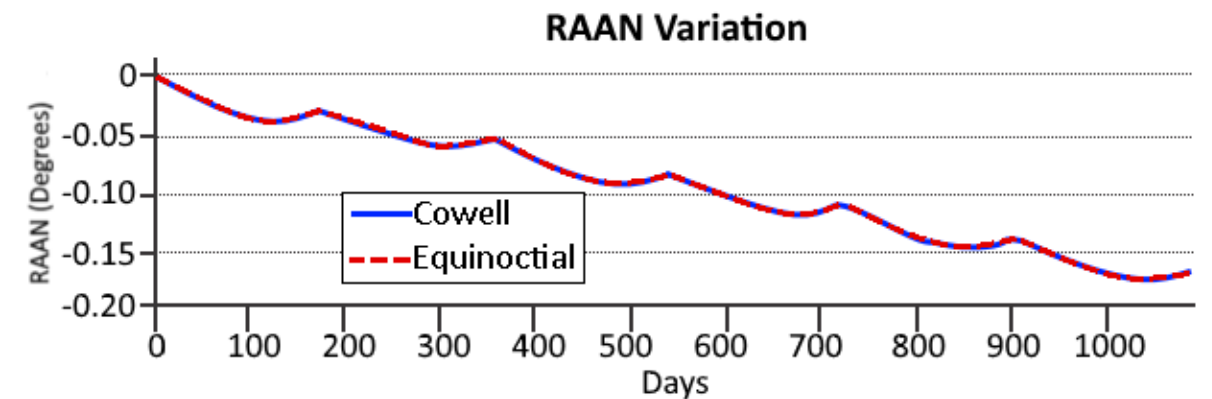

Arguement of Periee Variation

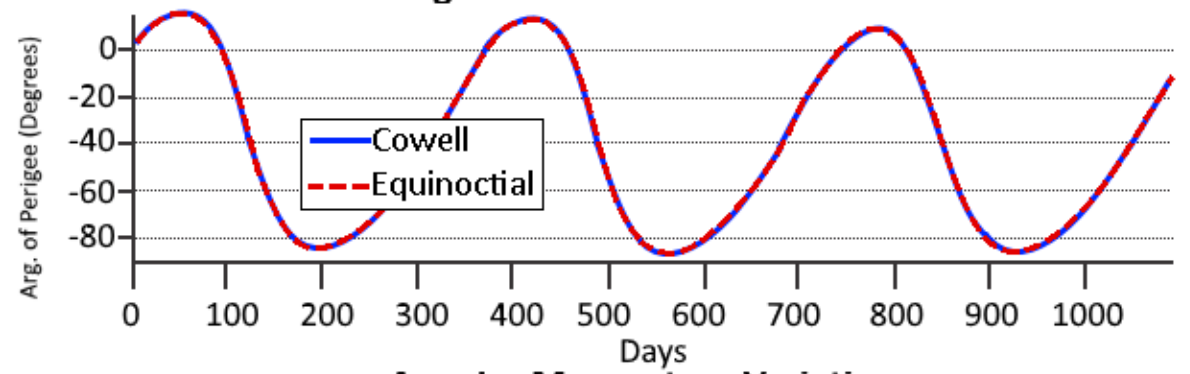

Angular Momentum Variation

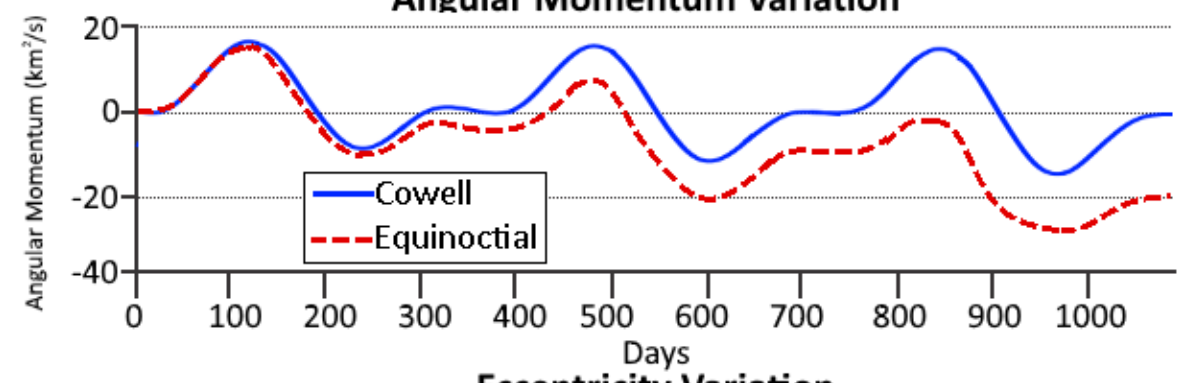

Eccentricity Variation
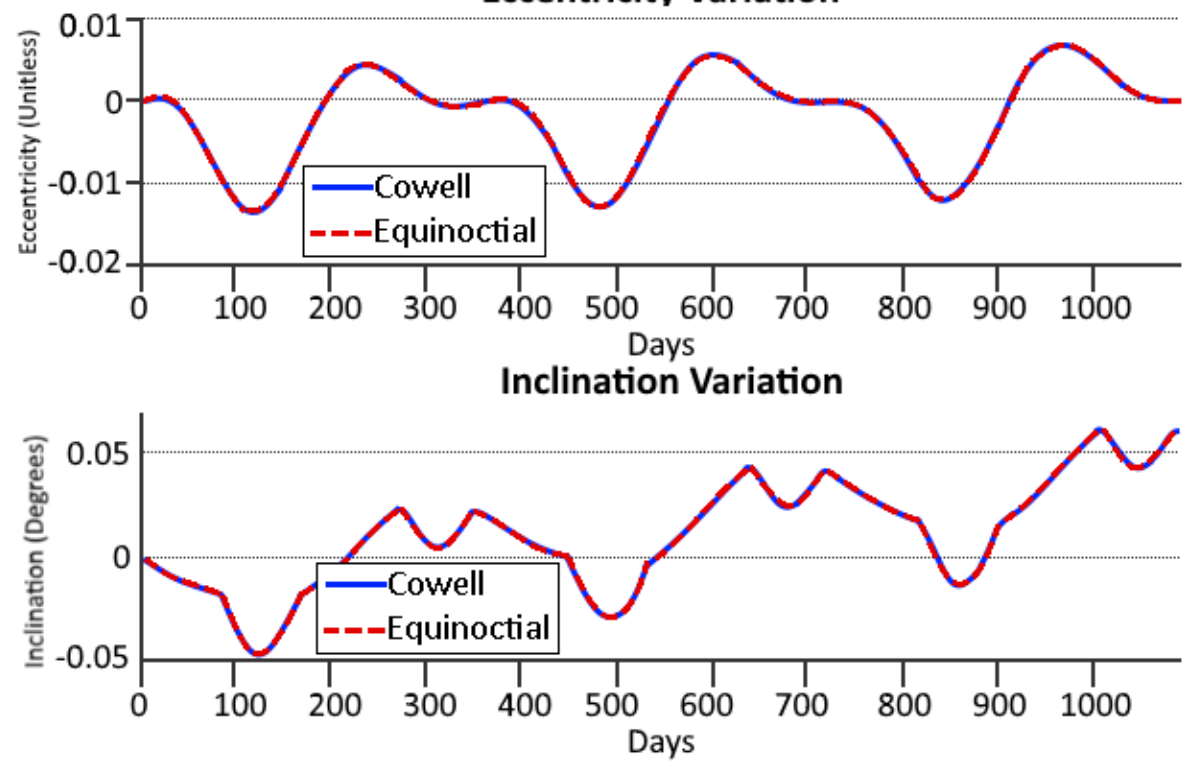

Figure 4.12: SRP Variation in Curtis, Example 12.7 


\subsubsection{Comparison Plots - TLEs, STK, and Cowell 3x3}

After validating the Cowell method output with success, the final sets of plots comparing performance against TLE data will be analyzed. These plots include Cowell output, STK output, and the data points of each satellite's TLEs. TLE data for the longitude and latitude of the object are determined using the data of each TLE and generating a state vector, then converting as is done with the other two sets of data. As TLEs are notoriously inaccurate, each set of TLE data was pre-processed by hand to remove anomalous, singular outliers in the data that did not reflect the actual behavior of the object. The removal of these points had no effect on the overall trend of the data and is simply done to visually clean the plots for presentation purposes. All historic TLE data presented here can be retrieved freely from Celestrak [18].

The first plots in Figure 4.13 are for object 03432. Looking to the long term plots, all custom trends match well with the TLE data except for the longitude. For all satellites, the longitude output of the TLEs will be a problem. This is likely a result of the limitations of the TLE data, as this object is a known drifter yet the TLE does not show this behavior.

It is important to note that the longitude calculations are strongly related to the true anomaly of each object. The true anomaly fluctuates quickly throughout the orbit, even quicker than the longitude does here, but this rapid fluctuation coupled with the low sampling frequency of TLE data results in a plot that, compared to the custom data, looks very similar to the longitude results. Looking at the true anomaly at each time step, each individual point usually falls along the custom trends, but the overall trend looks entirely different. To illustrate this point, an image is included in Figure 4.14. The blue line represents the actual behavior of a function, while the red dots are data points collected on the function at various points in time. The dotted line is the trend surmised by an observer only aware of the selected red data points. By taking measurements at a low sampling rate, the actual trend of the data is not captured and a misleading characterization can be applied to the behavior of the data. This same effect is happening to some degree in the longitude information generated using the TLE of the objects. This does not account for all of the inaccuracies of the TLEs, but it is one otherwise unexpected source of error. For this reason, the 
outcome of the TLE longitude will be disregarded when attempting to classify the accuracy of the simulation trends, but the other data displayed in the plots is not tied with such a hard-to-experimentally-determine characteristic of the orbits and is of merit in validation. As the longitude behavior is already known for each object, this does not cause a problem for the validation process.
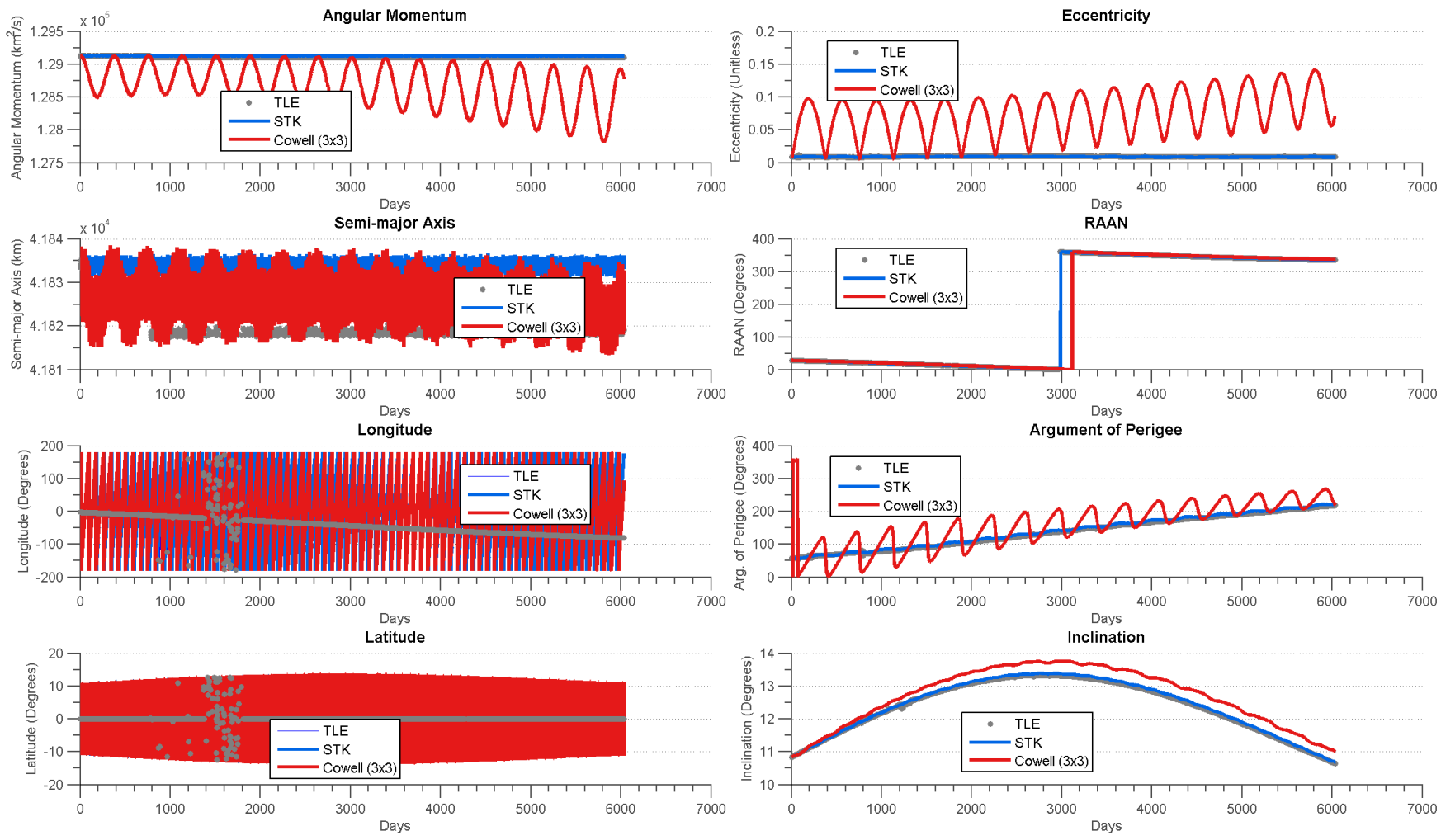

Figure 4.13: Long Term Plots for Satellite 03432

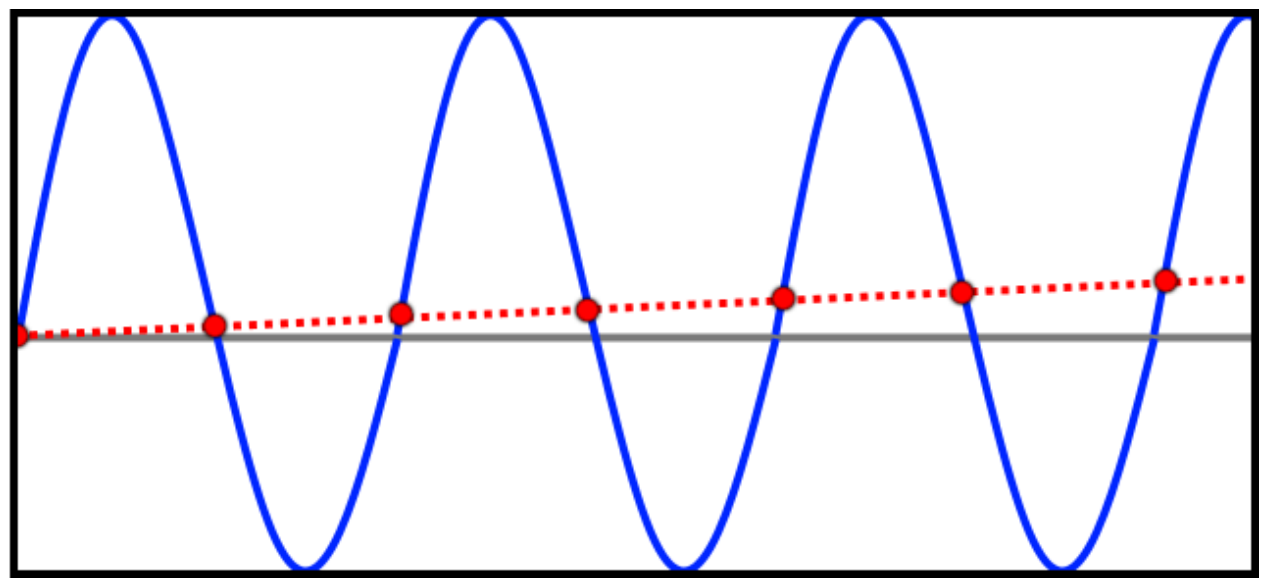

Figure 4.14: Longitude trend underlying behavior 
The plots for the ATS 5, satellite 04068, are shown in Figure 4.15. This satellite is one of the drifter objects identified in Table 4.1. Once again, the longitude does not match between the TLE data and calculated outputs, but all other descriptors of the orbit match. With satellite 04068 and satellite 03432 , it has been proven that drifting objects are well accounted for using a Cowell propagator with the selected perturbations. This is important, as the majority of GEO debris falls within this category.
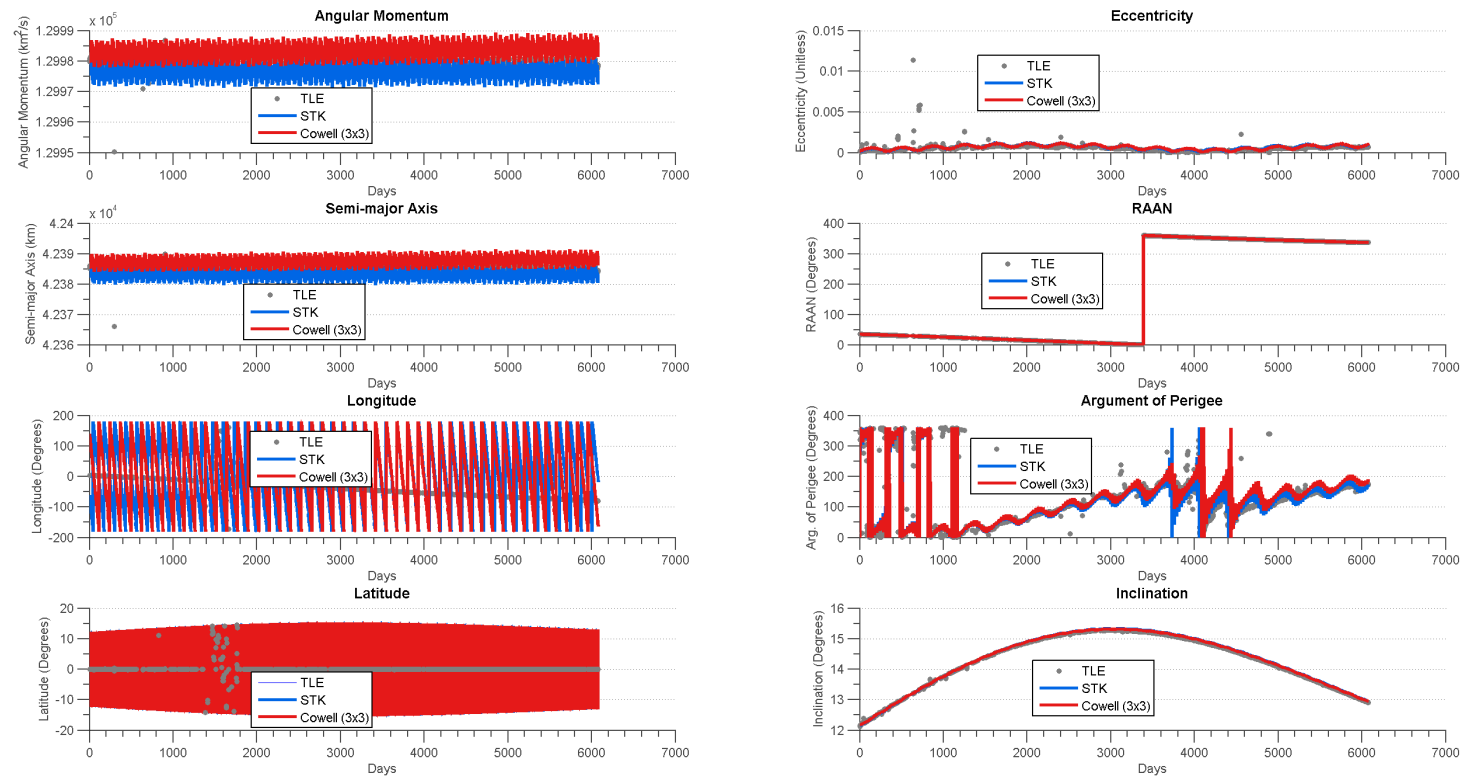

\section{Figure 4.15: Long Term Plots for Satellite 04068}

The output for Skynet 1, satellite 04250, is shown in Figure 4.16. This is a West well trapped object, and that longitudinal trapping is shown with the custom propagator. Both STK and Cowell output match well to the TLE data available,with the inaccuracies of Cowell being in the argument of perigee and the eccentricity. The assumption is that with an expanded set of geopotential perturbations these variations should be minimized and match the STK output. 

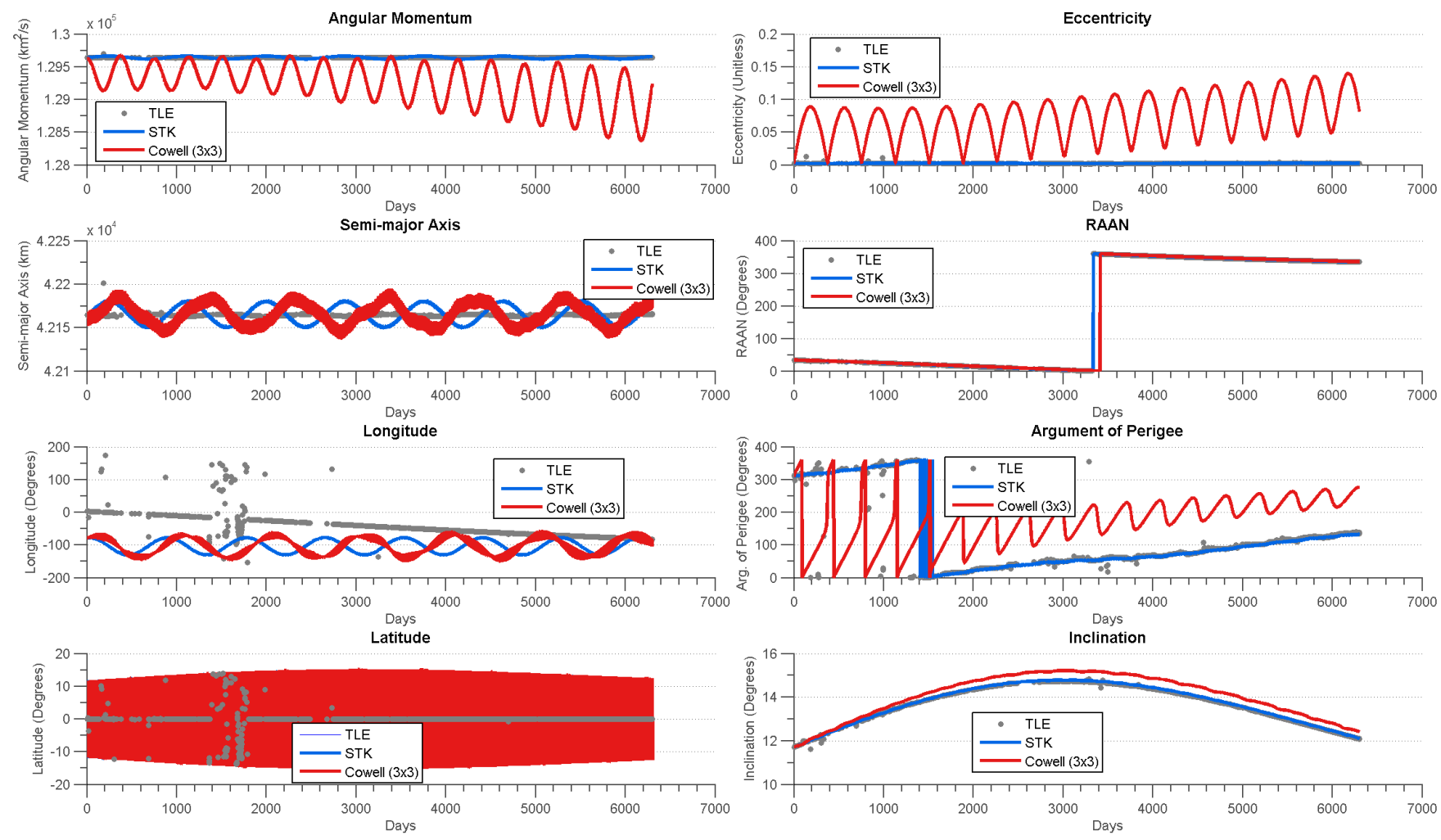

Figure 4.16: Long Term Plots for Satellite 04250

Satellite 05588, also known as OPS 9432, is the EW-trapped debris object displayed in Figure 4.17. Once again, both STK and the custom propagator meet expectations, though both lag behind the observational data represented with the TLEs in terms of angular momentum and semi-major axis. This is the second assurance that the custom propagator is successfully identifying EW-trapped behavior. 

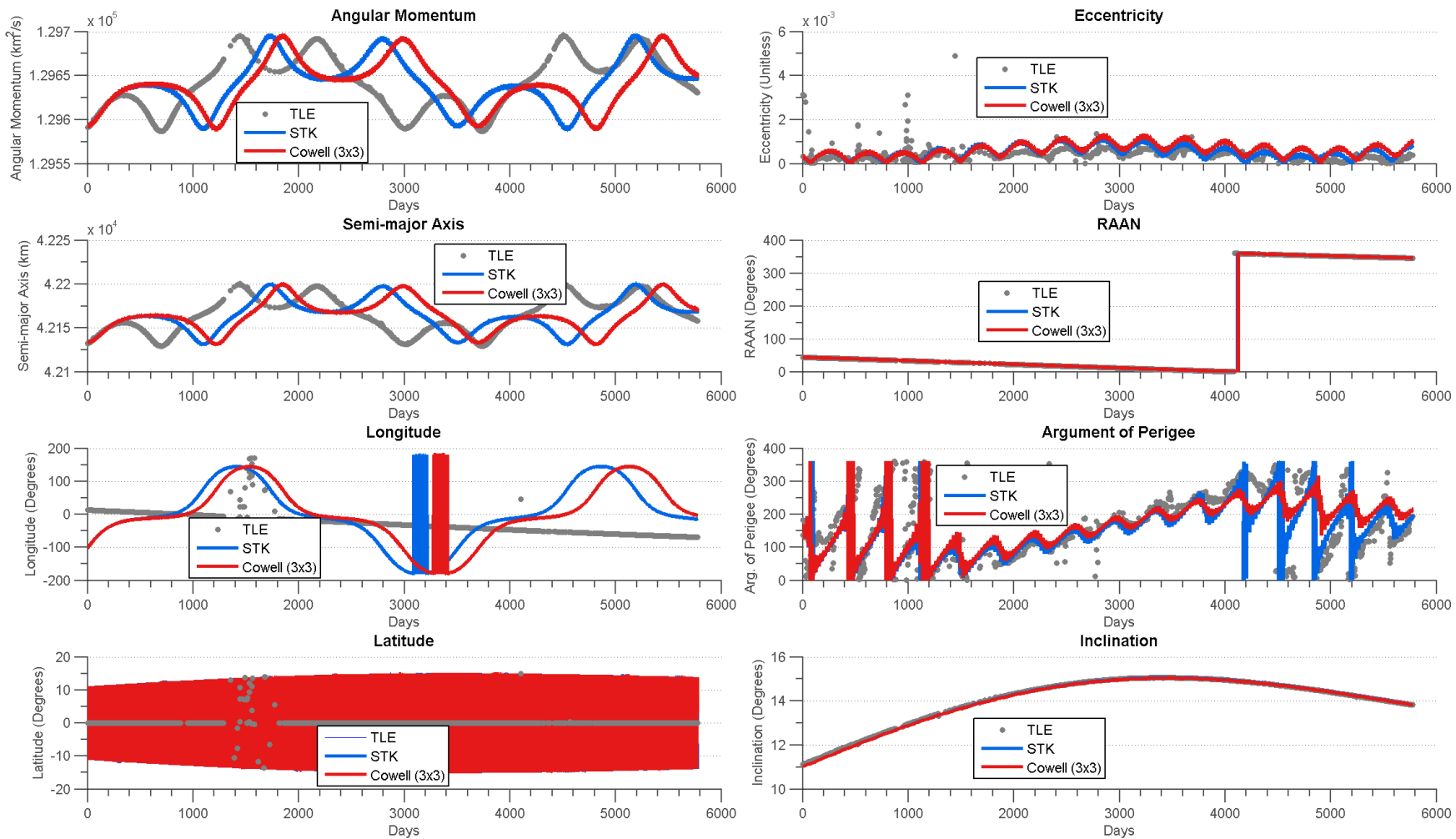

Figure 4.17: Long Term Plots for Satellite 05588

The final object being considered, satellite 29640, is the Eastern Well trapped object. The related plots are shown in Figure 4.18. In this comparison the computed methods match well with the STK data. The angular momentum, semi-major axis, and eccentricity all seem to differ, but looking at the scales of the plot it is clear that these are minute differences. The argument of perigee is the only strange behavior, and the TLE behavior can likely be linked to the near-zero eccentricity reported. At zero eccentricity, the argument of perigee becomes undefined and this phenomenon may explain the strange trends. Additionally, the discussion included in Figure 4.14 is illustrated well in the latitude of this satellite, which according to the TLE looks to oscillate much more slowly than in actuality.

At this point, the custom Cowell propagator utilizing the $3 \times 3$ geopotential, solar and lunar gravity, and SRP perturbations has been validated. This validation was conducted against STK, the TLEs, and the known expected longitudinal behavior of each satellite. In summary of the validation, a few key points have been made. First, the Equinoctial propagator was unsuccessfully implemented and the likely culprit is 

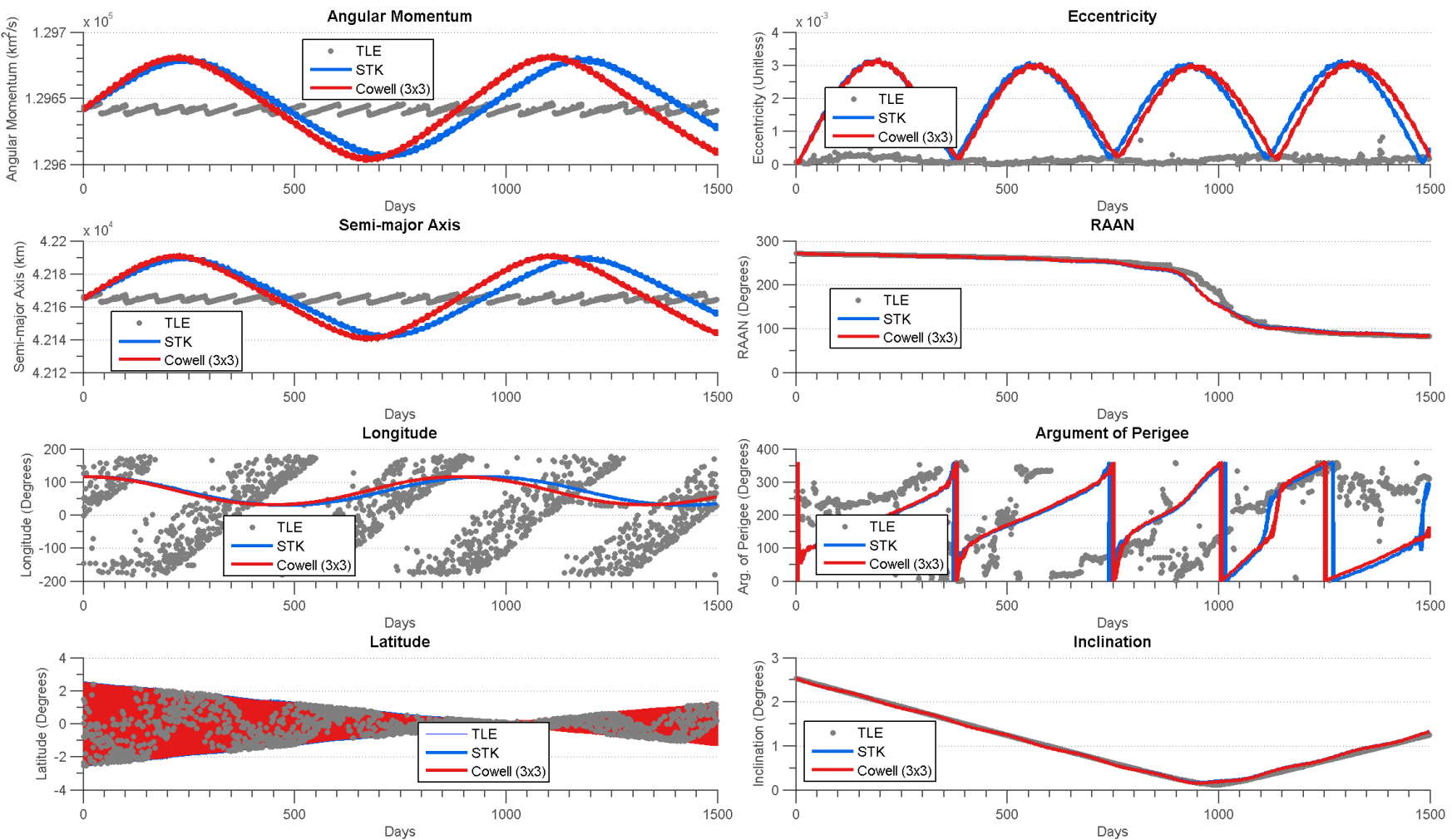

Figure 4.18: Long Term Plots for Satellite 29640

that an incorrect set of equations were used to express the equations of motion in the equinoctial frame. Second, the $J_{2}$ and $J_{22}$ terms did not contain the full set of required geopotential terms to successfully model all forms of well entrapment. By expanding this perturbation to include terms up to the $3 \times 3$, a reasonable approximation was produced for each type of well interaction. The Cowell method was also validated as an acceptable propagation method for the identification of expected geopotential well behavior. With a sufficient capability shown in modelling the expected sets of geopotential behavior, the collision simulation can now be validated before a final set of collision results are produced.

\subsection{Collision Simulation Validation}

Before being used to create results, the collision simulator must be validated against the numerous trends provided by Hanada [13]. The first of the inputs for the collision simulator in this validation phase are two satellite states that result 
in a collision of about $300 \mathrm{~m} / \mathrm{s}$ to match the average collision being represented in these low-velocity breakups. Additionally, two masses of $1000 \mathrm{~kg}$ and $400 \mathrm{~kg}$, and a parameter to generate at least 1,000 fragments are used. Fragments are generated from the largest characteristic length allowed for a piece of debris, down to smaller and smaller sizes until the minimum number of fragments specified is met. For further information regarding the distributions used to define the fragment characteristics, see chapter 3.

The resultant $\Delta v$ distribution is presented in Figure 4.19. Hanada's work shows that the $\Delta v$ distribution of a non-catastrophic breakup will result in an inverse cumulative distribution heavily weighted between 0 and $10 \mathrm{~m} / \mathrm{s}$. This figure agrees with Hanada's work, and using this data, the population of fragments can be generated.

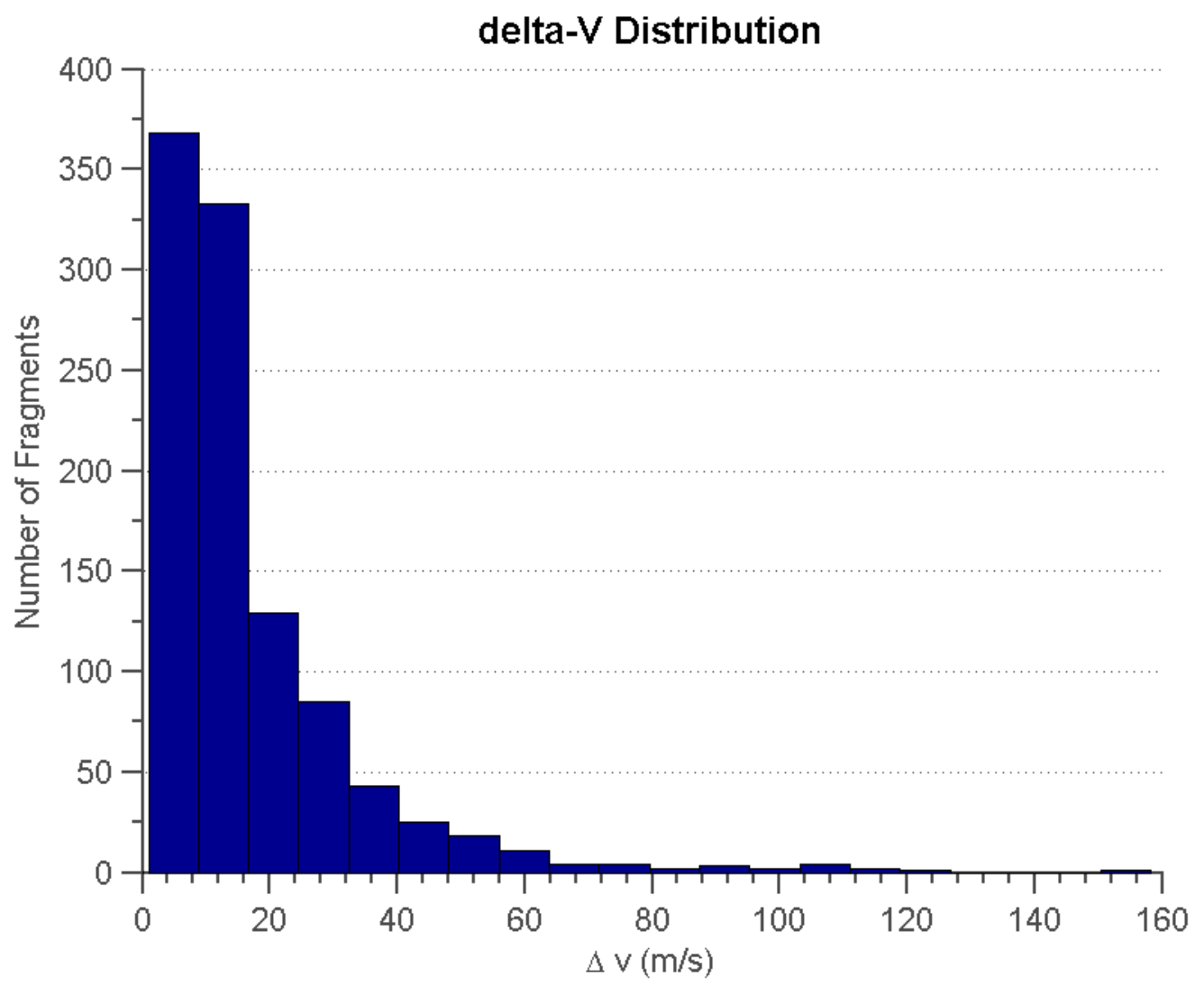

Figure 4.19: The $\Delta v$ distribution of fragments for two objects of $1000 \mathrm{~kg}$ and $400 \mathrm{~kg}$ during a $300 \mathrm{~m} / \mathrm{s}$ collision

Each fragment uses the same initial starting location, the location of collision, and the velocity vectors are determined as a sum of the largest object's velocity vector 
and a $\Delta v$ vector whose magnitude is taken from the distribution and unit vector determined via Gaussian distribution. Each fragment is also assigned its related $\mathrm{A} / \mathrm{m}$ ratio for use in SRP perturbation calculations. This process is broken down in Figure 4.20.

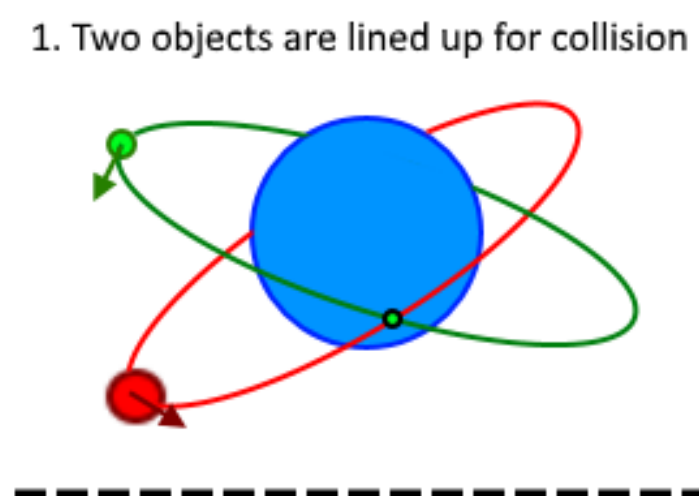

3. Gaussian distribution applied to delta-v results to generate new v's

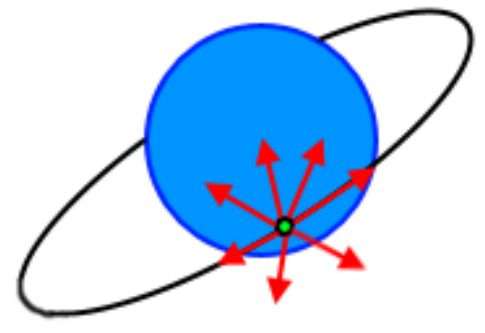

2. Collision Simulator uses inputs to generate distributions

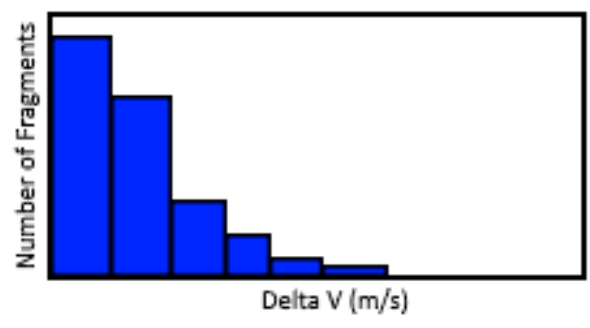

4. Generate and propagate new objects

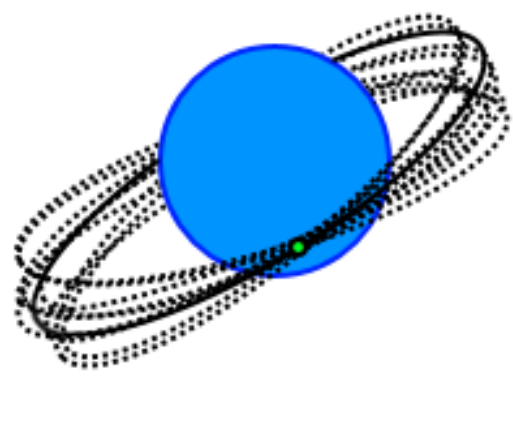

Figure 4.20: Conceptual outline of collision simulator usage

\subsection{Combined Simulation Results}

Most of the parent satellites used for the collision simulation are identified in Table 4.1, with an additional parent satellite belonging to a graveyard orbit. Using these parent satellites, a collision resulting in 1000 distinct pieces of debris fragments will be carried out. From each of these collisions, the slowest and fasted child objects will be chosen for analysis, which should help in understanding the full spectrum of behavior belonging to fragments of a collision. The slowest object is considered the fragmentation object that is imparted with the smallest change in $\Delta v$ from its parent, while the fastest object receives the largest $\Delta v$. The collision will be assumed to occur 
at the initial point of analysis in prior propagations so that the longitudinal behavior of the children objects can be compared to the base behavior of their parents.

For each of the satellites and their child fragments, the $\Delta \Delta$ over time was calculated along with the longitudinal behavior. The $\Delta \Delta$ term is determined as in Equation 1.2 and is used to identify if an object is trapped in a geopotential well. The results of the $\Delta \Delta$ plots corroborate with each of the longitudinal results, so they will not be presented here.

Longitudinal fragmentation behavior is shown for satellite 04068 in Figure 4.21. As a drifting satellite, it is unlikely for a child object to become trapped unless the collision occurs next to a well and enough of a change in the apoapsis and periapsis was observed to meet the $\Delta \Delta$ requirement expressed previously in Figure 1.3. The results shown that capture is not achieved for the object.

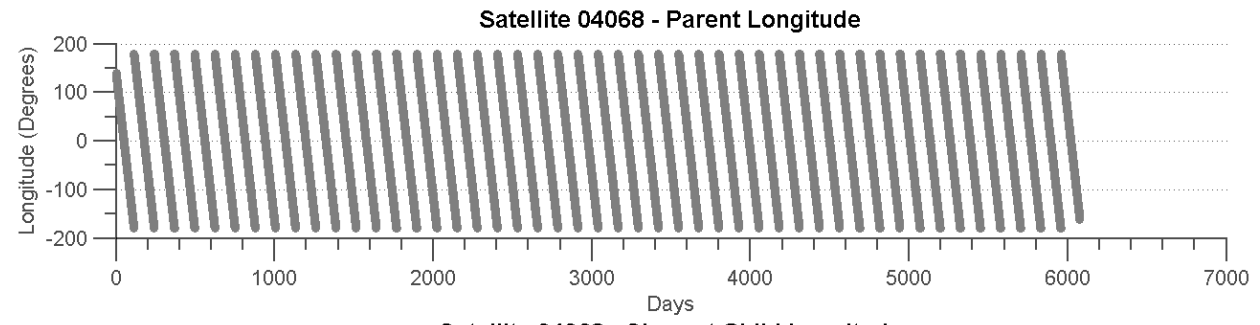

Satellite 04068 - Slowest Child Longitude

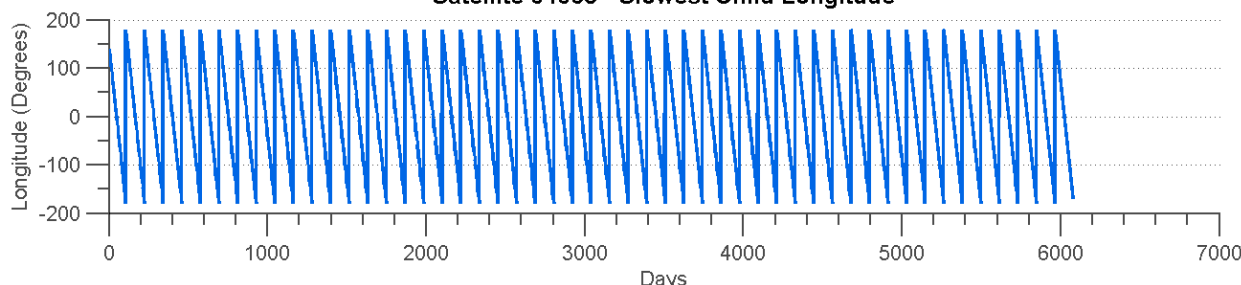

Satellite 04068 - Fastest Child Longitude

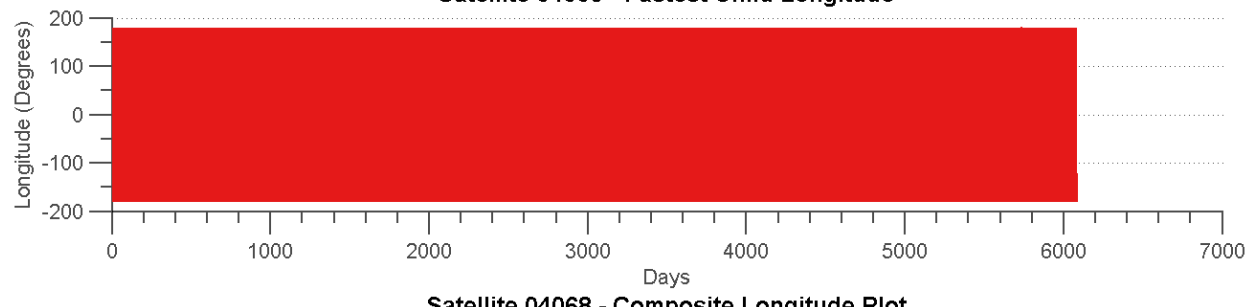

Satellite 04068 - Composite Longitude Plot

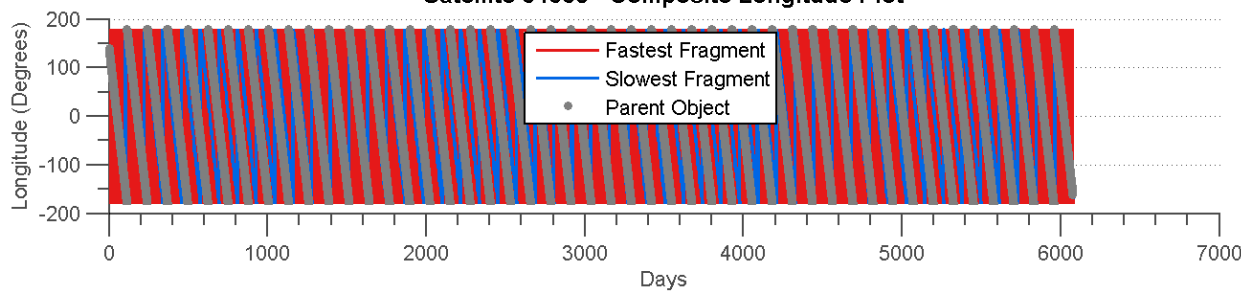

Figure 4.21: Longitudinal Fragmentation Behavior - Satellite 04068 
The longitude behavior for satellite 04250 is provided in Figure 4.22. Interestingly, the fast object achieved enough of a boost in velocity to escape the East well entrapment that its parent suffered. It should be expected that with other collisions taking place in the Eastern well, there is indeed a potential for fragments to escape the well and potentially impact the GEO regimes safety. If the fastest object achieves such a strong drifting behavior, it can be inferred that over the given range of longitudinal behavior from East entrapment to drifting, fragments could also become EW trapped. This range of behavior means a large degree of motion is available to the resulting fragments of an Eastern well collision, and future care should be taken if such a collision were to occur.
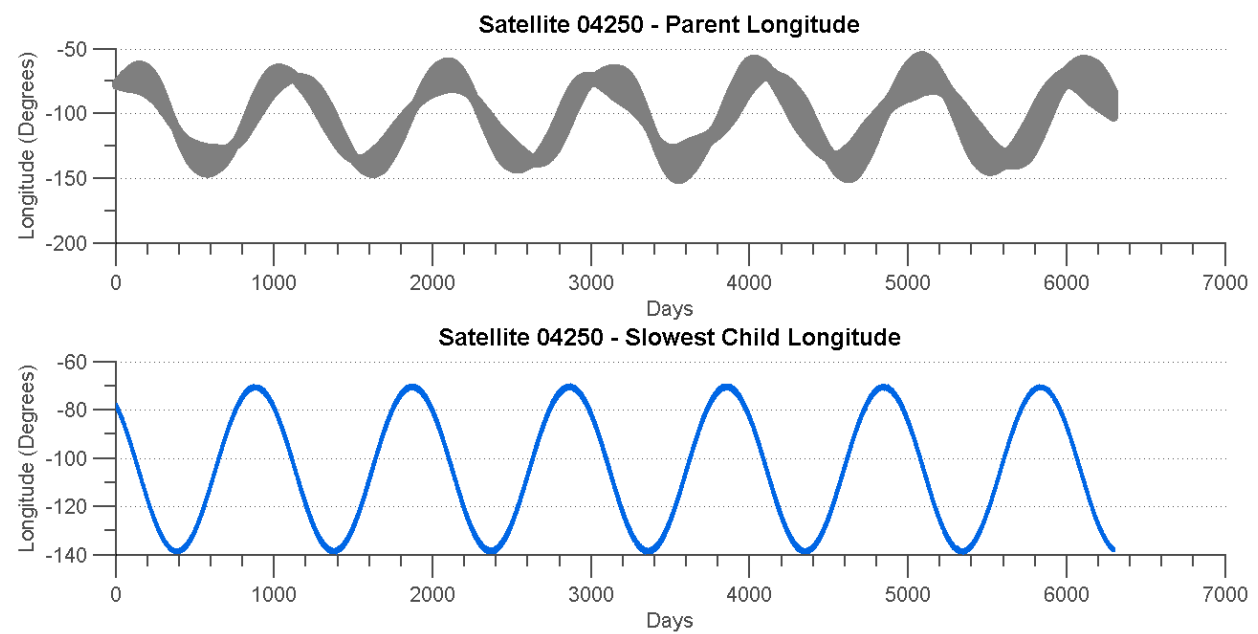

Satellite 04250 - Fastest Child Longitude

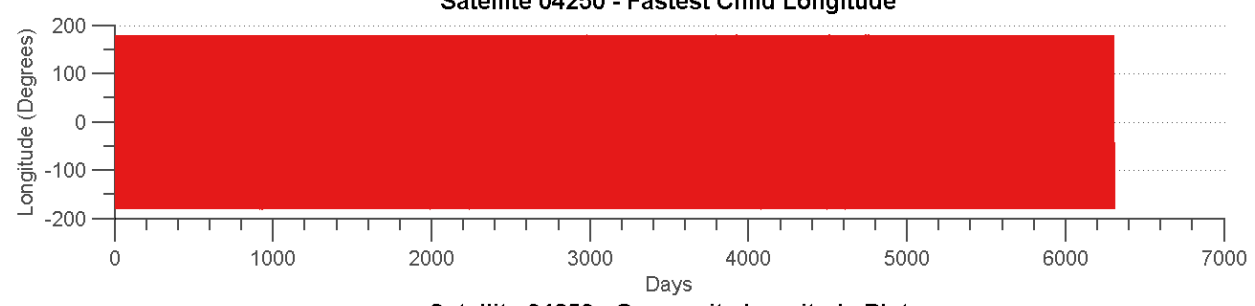

Satellite 04250 - Composite Longitude Plot

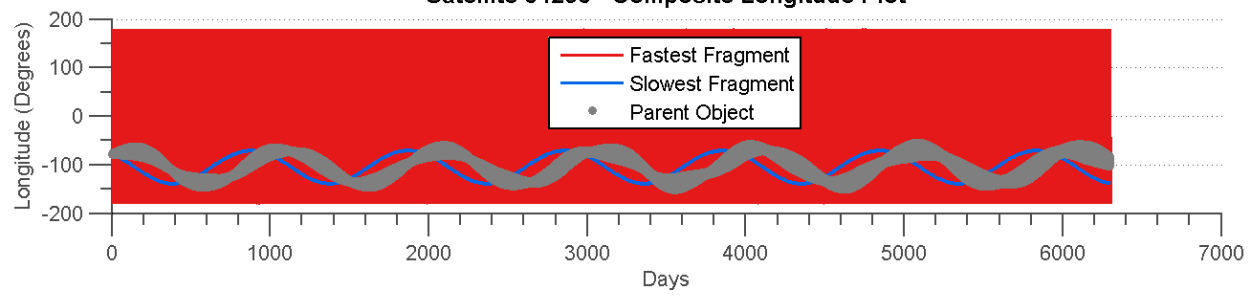

Figure 4.22: Longitudinal Fragmentation Behavior - Satellite 04250

Satellite 05588 is the EW-trapped representative for the longitude fragmentation behavior analysis and its results appear in Figure 4.23. EW-trapped objects are bal- 
anced carefully between drifting and singular-well entrapment, so slight $\Delta v$ changes are likely to result in changes in longitude. Both slowest and fastest objects have achieved a drifting behavior in this case. A future expansion upon this would would be to understand whether child objects in these cases can be shifted to singular entrapment. Additional cases and a full analysis of fragmentation behavior should be looked to in order to answer this question.
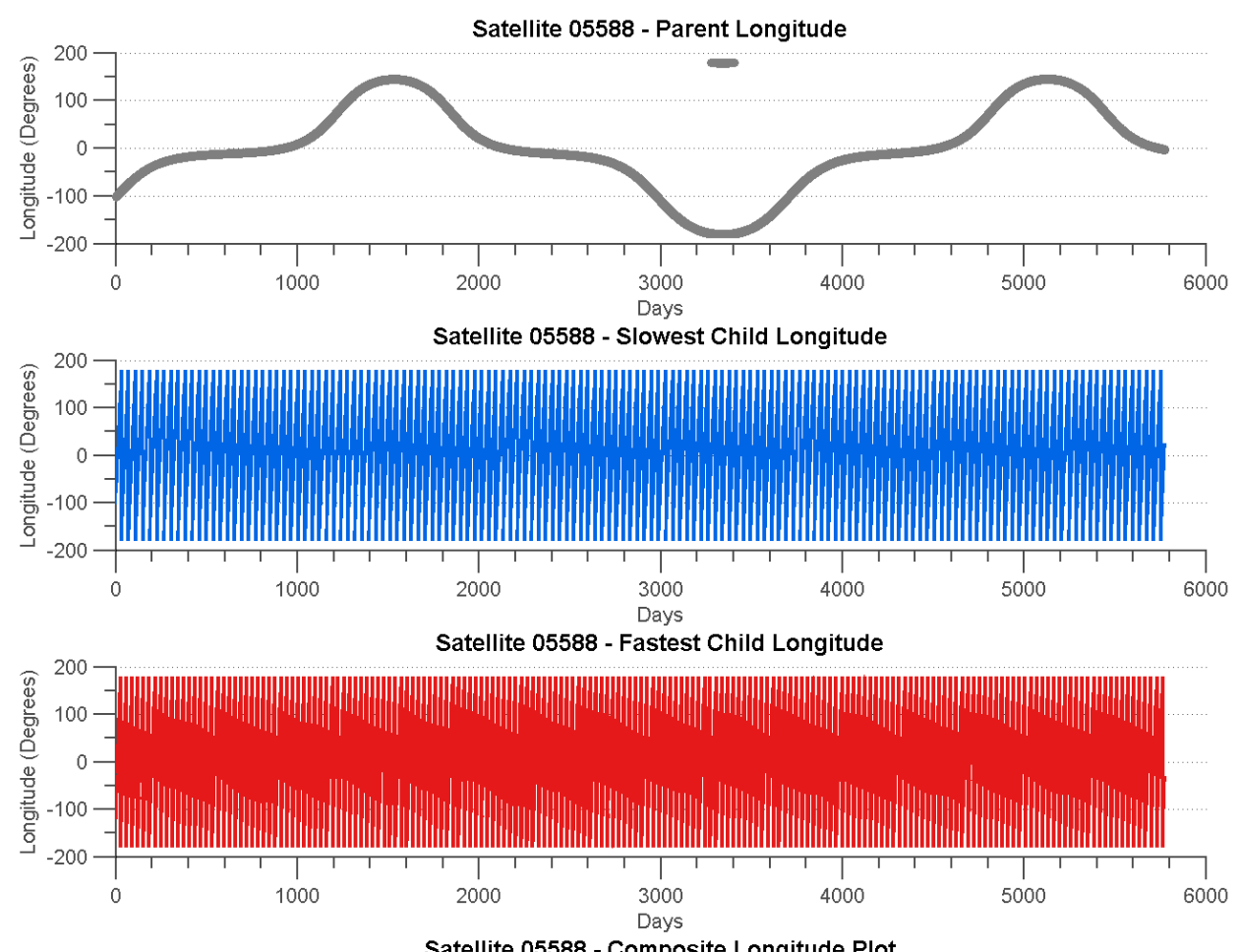

Satellite 05588 - Composite Longitude Plot

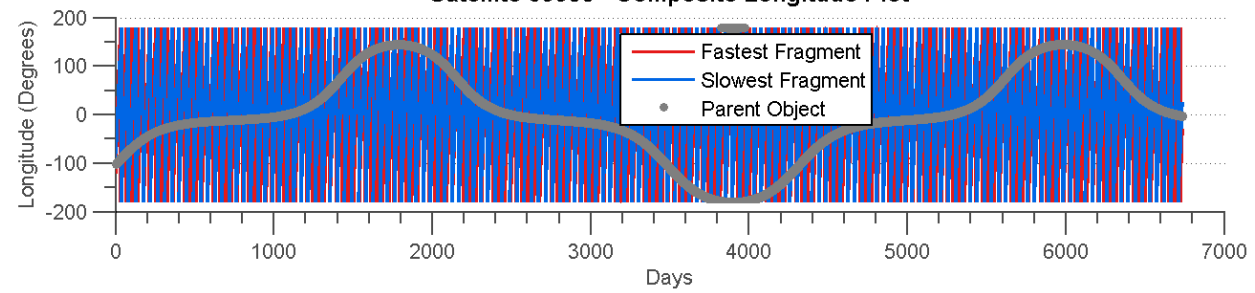

\section{Figure 4.23: Longitudinal Fragmentation Behavior - Satellite 05588}

Figure 4.24 displays the behavior of Satellite 29640's fragmentations. The parent object is a West-well trapped object, and similarly to the East well, the fastest object breaks from the well and becomes a drifting object. Though the well is though to be a sink, collisions within the well have potential to escape and, over twenty years, never become captured again. The slow fragments maintain the trapped longitudinal behavior of the parent object however. As with the East well, these results signal 
that collisions within the wells can be cause for concern to other objects in the GEO belt.
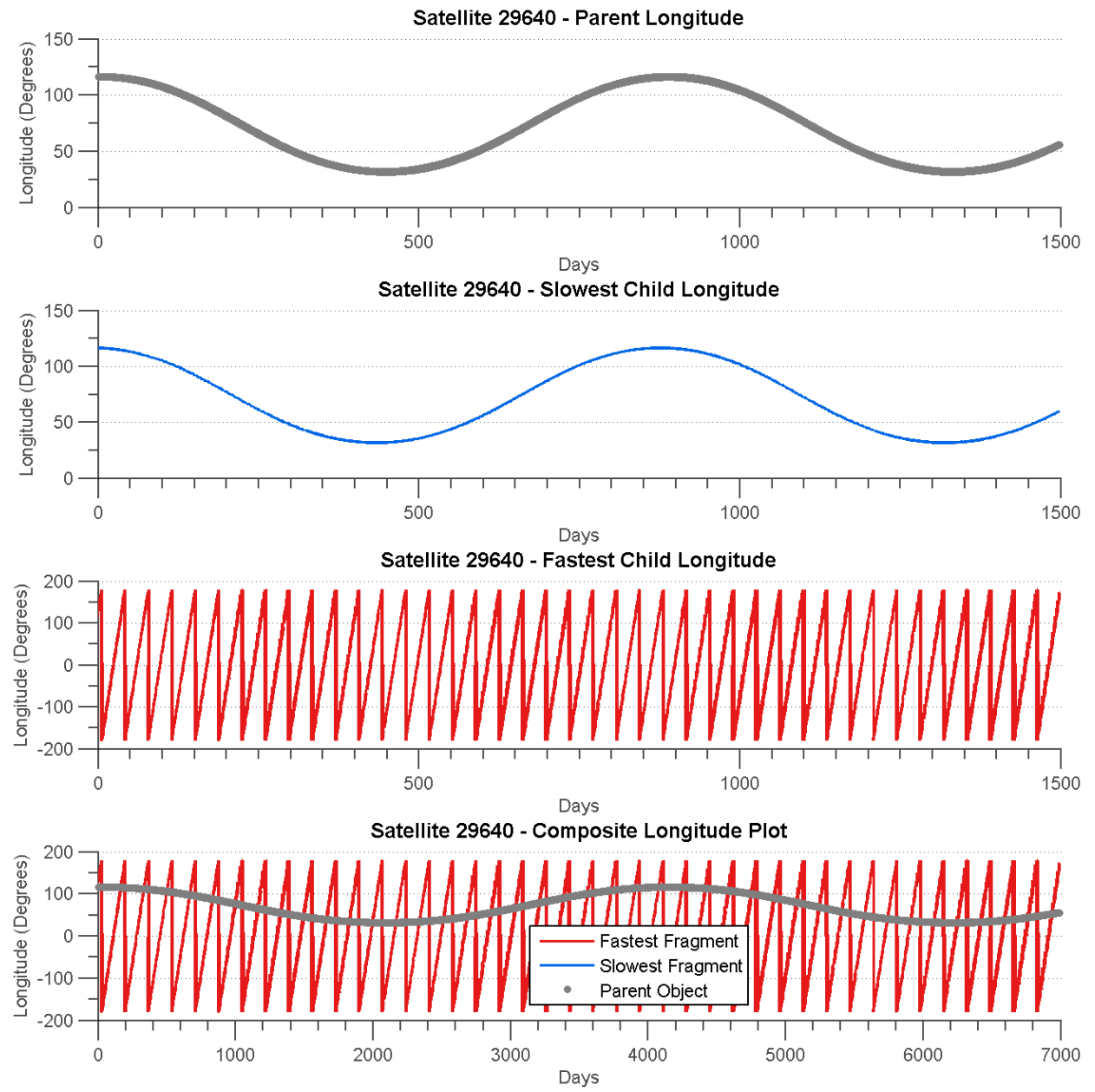

Figure 4.24: Longitudinal Fragmentation Behavior - Satellite 29640

The final object of analysis is shown in Figure 4.25. This object is not linked to a real peice of debris, but was created for this analysis and is defined by the required perigee and apogee of a standard graveyard orbit object. The COEs defining this objects initial state are provided in Table 4.2. 
Table 4.2: Graveyard Object State

\begin{tabular}{|c|c|}
\hline State Term & Value \\
\hline Julian Date & 2454087.24 \\
\hline Angular Momentum $\left(\mathrm{km} / \mathrm{s}^{2}\right)$ & 232113.0 \\
\hline Eccentricity & 0.0823 \\
\hline RAAN (deg) & 327.4513 \\
\hline Arg. of Periapsis (deg) & 56.3244 \\
\hline Inclination (deg) & 0.0057 \\
\hline True Anomaly (deg) & 58.8411 \\
\hline
\end{tabular}

Graveyard orbit objects are expected to act like drifting satellites, which the parent object conveys. This case is studied however to determine if, following a collision, there is a possibility for a standard graveyard object to behave in such a way that it endangers the GEO belt. The child objects of the simulated collision event confirm this, showing that this object poses no threat. A $\Delta \Delta$ analysis was done for this set of debris, but because the apogee and perigee are distant enough from the GEO belt these fragments are all mean a drifting object classification.

In the case of the graveyard orbit, the semi-major axis and eccentricities were additionally considered. At no point do these values drop to a point that would indicate danger to the GEO belt of satellites. After the initial collision, these values were stable and never displayed erratic behavior. Continued investigation however on a possible collision of a graveyard satellite and an object transitioning from GEO would be of benefit. 

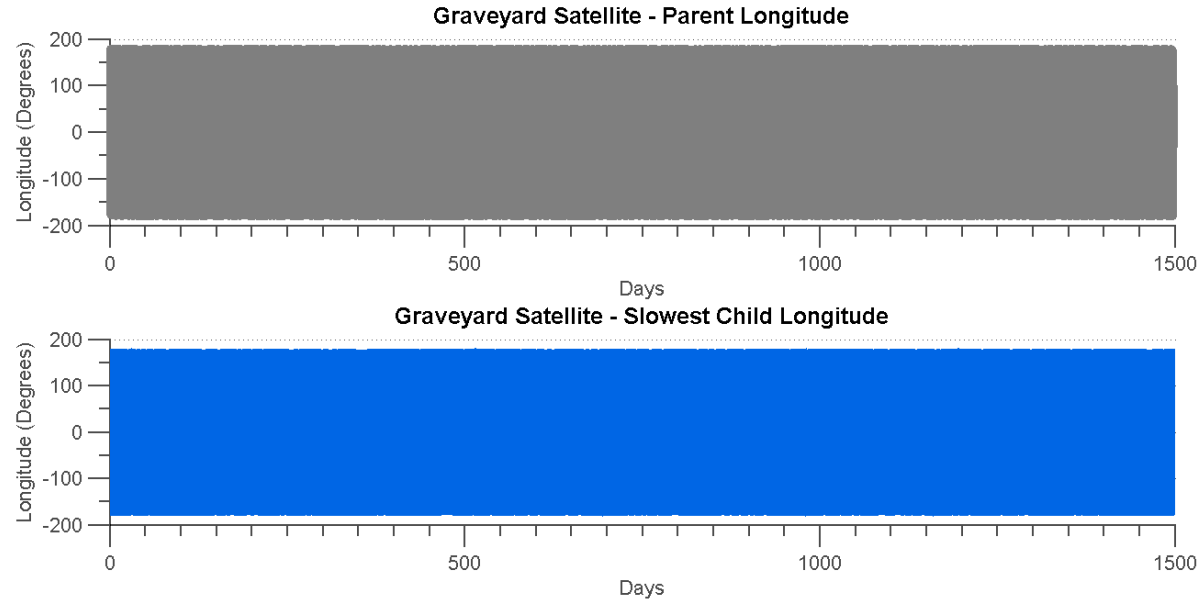

Graveyard Satellite - Fastest Child Longitude
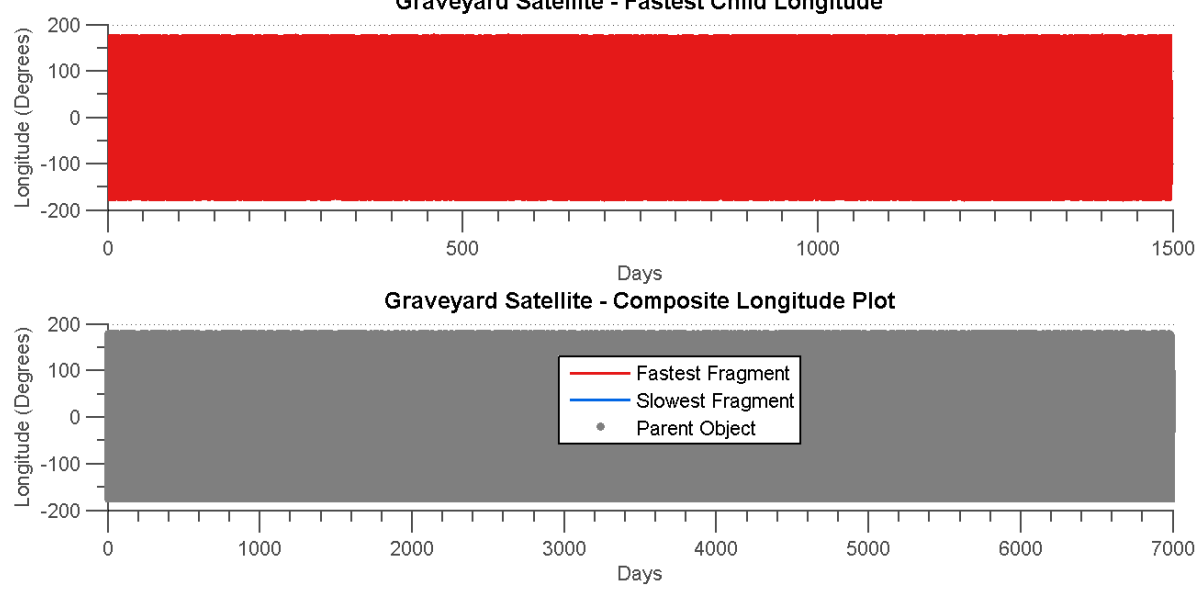

Figure 4.25: Longitudinal Fragmentation Behavior - Graveyard Satellite 


\section{Chapter 5}

\section{Conclusion}

Two MATLAB propagators were developed, one using a Cowell method approach and the other a Variation of Parameters method with Equinoctial Elements. These propagators were tested against the STK 10 propagator and observational TLE data using a selection of spacecraft debris in the GEO and graveyard orbits. It was determined that the Equinoctial VoP propagator faced an issue in properly implementing the solar radiation pressure at these regimes. The Cowell propagator succeeded in the validation phase for the modelling of all four types of previously identified GEO debris behaviors: drifting, East-well trapped, West-well trapped, and EW-trapped. It was discovered that in order to capture the EW-trapped behavior, the use of additional geopotential perturbations was needed alongside the original proposed $J_{2}, J_{22}$, Sun, Moon, and SRP perturbations. Based on this, the use of a simple Cowell propagator would be more than enough to model the behavior of GEO debris for over 20 years of analysis. Limitations in the Cowell's method accuracy were identified in the results analysis, but it is suspected that these inaccuracies would diminish if additional perturbations are introduced. Additional tesseral harmonics and sectoral harmonics would be the first place to start improving the perturbations, followed by more accurate SRP and third-body effects. Selecting a higher fidelity model to approximate the Sun's position will help to further increase accuracy in both third-body and SRP calculations.

This thesis builds upon prior analysis conducted by Christina Diaz [8]. Diaz created a Cowell propagator for geopotential well analysis, but the propagator was unable to model EW-trapped objects. The assumption drawn from this was that 
the Cowell propagator was too inaccurate to be capable of modelling the complex dynamics of this entrapment. Additionally, it was suggested that the use of a highly complex and dynamic area calculation may be needed in the SRP analysis in order to meet the accuracy requirements needed from the perturbative forces. The results presented in this paper however, prove that the Cowell propagator is entirely capable of simulating the EW-trapped behavior. This behavior is achieved using only the simple spherical cross-sectional area and reflection models for the solar radiation pressure. The minimum required set of geopotential terms and other perturbations needed to simulate all modes of entrapment was identified. This was found to be geopotential terms up to 3x3, solar radiation pressure, and the third-body Sun and Moon perturbations. Because of this capability, the collision analysis was able to be extended to the EW-trapped case unlike previously.

Due to the large difference in the longitudinal behavior and other classical orbital element plots in this paper and that produced by Diaz, the likely source of error in the latter paper is tied to the perturbation modelling. The individual effects of the Sun, Moon, SRP, and $J_{2}$ perturbations have numerous published examples to compare against when validating propagators. It is not as simple to find these comparison cases for the remaining geopotential perturbations however, and as shown in subsubsection 2.2.1.3, the equations are not simple to implement. The combination of a lack of good validation sources and the difficulty in accurately converting the equations into code identifies the most likely place an error could have occurred. While general trends in the other entrapment cases produced by Diaz matched the results shown here, the exact trends were not matched. An error in the geopotential terms may have resulted in small inaccuracies in each of the cases, but because of the very strict $\Delta \Delta$ requirements for EW entrapment, this particular case could not be successfully simulated in the propagator created by Diaz.

Five test cases were produced for validation, and these objects were then used in the generation and analysis of low velocity breakups for GEO satellites. A recreation of Hanada's low velocity breakup model was used to generate debris fragments and results were produced for each type of entrapment. In addition, a test case was run for a graveyard orbit object. The drifter and graveyard objects maintained their drifting state across their fastest and slowest breakup fragments. The West well object's 
slowest fragment continued to be trapped, but the fastest adopted a drifting behavior. The East well behaved similar to the West well, with the fastest object escaping the influence of its well and becoming a drifter. As the $\Delta \Delta$ increases or decreases for a trapped object, it passes through the EW-entrapment state before reaching the drifting state. This means that if the fastest object from the East and West well collisions achieved a drifting state, it is possible that slower breakup fragments could become EW-trapped. Finally, the EW-trapped object's slowest and fastest fragments both achieved a drifting state. It is clear from these results that future care should be taken when considering collisions within the geopotential wells, as fragmentation will have a potential for great mobility. A collision with enough force between objects can create fragments which may escape their origin well and potentially interact with the greater GEO spacecraft population.

\subsection{Future Work}

Future work in this topic would benefit from a number of lessons learned during this thesis. The first is that a Cowell method propagator is more than capable of accurately simulating GEO debris behavior, provided the correct perturbations are used and computational efficiency is noncritical. If efficiency is key, Cowell's method should be replaced with a Variation of Parameters method, and the Equinoctial element set avoids the singularities at zero eccentricity and inclination that other $\mathrm{VoP}$ methods face. However, it is advised that prior to the use of Equinoctial elements, the user derives the equations themselves as a number of inconsistencies have been identified across the most widely available papers on the subject. To simulate well entrapment, the most important perturbations are the geopotential terms up to $J_{33}$. Tesseral terms are a necessity to capture East-West well behavior, and Sectoral harmonic $J_{22}$ is important in capturing long-term GEO-specific behaviors. Geopotential perturbations play a larger role in modelling this behavior than other perturbations, but for a strong analysis, the inclusion of solar and lunar gravity, as well as solar radiation pressure, are needed. Future work should consider expanding on the analysis of well fragmentation: it was determined that fragments can escape their wells, but it would be worth examining what behavior an entire population of fragments adopt. 
It has yet to be determined if one of these well collisions has a possibility to achieve EW entrapment, or what conditions specifically allow for fragments originating from a trapped object to leave their well. Additional satellites of interest for this study, as well as discussion on experimental observations on the subject, can be found in [20].

MATLAB was a limiting factor as its computational efficiency slows the generation of results, but its ability to rapidly prototype the initial design and the visibility it offers of its variables helped greatly. To improve future efforts to recreate this analysis, it is recommended that once the propagator has been validated in a rapid prototyping language such as MATLAB, a more computationally efficient language should be used to recreate the code. Typical MATLAB simulations of a 20 year propagation using Cowell's method should be expected to take at least one hour, and STK computations with the perturbations identified in the results will take two hours to generate. Long-durations tests also require the user to increase the memory available to the MATLAB IDE, otherwise memory must be carefully managed to avoid crashes. A typical output for the specified Cowell propagator results in nearly 30 million data points when considering the COEs, latitude, longitude, and time outputs. If an equinoctial propagator is constructed, the amount of resultant data is at least quartered. Where long-term propagations are required, there would be noticeable benefits in computational speed and memory usage if the equinoctial propagator could be successfully adopted. 


\section{BIBLIOGRAPHY}

[1] R. I. Abbot and T. P. Wallace. Decision support in space situational awareness. Lincoln Laboratory Journal, 16(2):297-335, 2007.

[2] R. H. Battin. An Introduction to the Mathematics and Methods of Astrodynamics. AIAA Education Series, 1999. Revised Edition.

[3] P. J. Cefola. Equinoctial orbit elements - application to artifical satellite orbits. AIAA/AAS Astrodynamics Conference, 1972.

[4] G. M. Clemence. Tiny earth satellites. Science News Letter, page 180, mar 1954.

[5] I. A. D. C. Committee. Report of the iadc activities on space debris mitigation measures. Online. 41th Session of the Scientific and Technical Subcommittee United Nations Committee on the Peaceful Uses of Outer Space.

[6] H. D. Curtis. Orbital Mechanics for Engineering Students. ButterworthHeinemann, third edition, nov 2013.

[7] J. C. V. der Ha. Long-term evolution of near-geostationary orbits. Journal of Guidance and Control, 9(3):363-370, may 1986.

[8] C. Diaz. A study of the collisional evolution of orbital debris in geopotential wells and geo disposal orbits. Master's thesis, California Polytechnic State University, San Luis Obispo, jul 2013.

[9] L. J. Friesen, A. A. Jackson, H. A. Zook, and D. J. Kessler. Analysis of orbital perturbations acting on objects in orbits near geosynch. earth orbit. Journal of Geophysical Research, 97(E3):3845-3863, 1992.

[10] A. S. Ganeshan. Simulation and modelling of orbital debris environment by equivalent breakups. Advances in Space Research, 19(2):309-312, 1997. 
[11] A. Guenther. Cal poly thesis template, jun 2016.

[12] L. Hall. 2014 roadmap to the stars. In The History of Space Debris. Space Traffic Management Conference, Embry-Riddle Aeronautical University, nov 2014.

[13] T. Hanada. Using nasa standard breakup model to describe low-velocity impacts on spacecraft. Journal of Spacecraft and Rockets, 42(5):859-864, sep 2005.

[14] G. R. Hintz. Survey of orbit element sets. Journal of Guidance, Control, and Dynamics, 31(3):785-790, may 2008.

[15] J. Hyde, E. Christiansen, R. Bernhard, J. Kerr, and D. Lear. A history of meteoroid and orbital debris impacts on the space shuttle. Proceedings of the Third European Conference on Space Debris, 1, 2001.

[16] J. H. Jo, I. K. Park, N. Choe, and M. Choi. The comparison of the classical keplerian orbit elements, nonsingular orbital elements (equinoctial elements), and the cartesian state variables in lagrange planetary equations with $\mathrm{j} 2$ perturbation: Part i. Journal of Astronomy and Space Sciences, 28(1):37-54, mar 2011.

[17] N. L. Johnson. A new look at the geo and near-geo regimes: Operations, disposals, and debris. Acta Astronautica, 80:82-88, may 2012.

[18] T. S. Kelso. Frequently asked questions: Two-line element set format, may 2014.

[19] D. J. Kessler. Sources of orbital debris and the projected environment for future spacecraft. AIAA, 18(4):357-360, jul 1981.

[20] R. I. Kiladze and A. S. Sochilina. On evolution of geostationary satellite orbits. Advanced Space Res., 19(11):1685-1688, 1997.

[21] P. H. Krisko. Nasa long-term orbital debris modeling comparison: Legend and evolve. 54th International Astronautical Congress of the International Astronautical Federation, the International Academy of Astronautics, and the International Institute of Space Law. Bremen, Germany.

[22] M. List, S. Bremer, B. Rievers, and H. Selig. Modelling of solar radiation pressure effects: Parameter analysis for the microscope mission. International Journal of Aerospace Engineering, 2015, 2015. 
[23] D. S. McKnight and F. R. D. Pentino. International astronautical congress. In New insights on the orbital debris collision hazard at geo, 2011.

[24] NASA. Monthly number of objects in earth orbit by object type. Orbital Debris Quarterly News, 21(1):12, 2017.

[25] O. of Science and T. Policy. Interagency report on orbital debris. Technical report, The National Science and Technology Council - Committee on Transportation Research and Development, 1995.

[26] N. O. D. P. Office. Frequently asked questions, 2016.

[27] U. N. A. Office. Astronomical Almanac for the Year 2013. Department of the Navy, 2013.

[28] J. A. Paris. The effects of using solar radiation pressure to alleviate fuel requirements for orbit changing and maintenance of the dscs ii f-13 satellite. Master's thesis, Department of the Air Force Air University, mar 2006.

[29] D. S. F. Portree. Orbital debris: A chronology. Technical report, NASA, jan 1999.

[30] S. T. R. Westerkamp Friesen, J. Bendisch Friesen and P. Wegener. Analysis of the space debris collision risk at geosynchronous altitude outside the equatorial plane. Advances in Space Research, 19(2):361-364, 1997.

[31] B. Romgens. Satellite collision avoidance prediction using verified interval orbit propagation. Journal of Guidance, Control, and Dynamics, 36(3):821-832, mar 2013.

[32] M. Rupp. The effects of j22 perturbation on geosynchronous orbit mission design, $\operatorname{dec} 2009$.

[33] N. Sorokin. Earth will drown in garbage. Voice of Russia, mar 2013. http://www.spacemart.com/reports/Earth_will_drown_in_garbage_999.html.

[34] T. Tamblyn. International space station hit by space debris causing tiny crack in window, may 2016. 
[35] D. A. Vallado. Fundamentals of Astrodynamics and Applications. Microcosm Press, fourth edition, 2013.

[36] T. Wang. Analysis of debris from the collision of the cosmos 2251 and the iridium 33 satellites. Science $\& 3$ Global Security, 18(2):87-118, 2010.

[37] B. Weeden. 2007 chinese anti-satellite test fact sheet. Online, nov 2010. https://swfound.org/media/9550/chinese_asat_fact_sheet_updated_2012.pdf. 


\section{APPENDICES}

\section{A. No-SRP Equinoctial Variation}
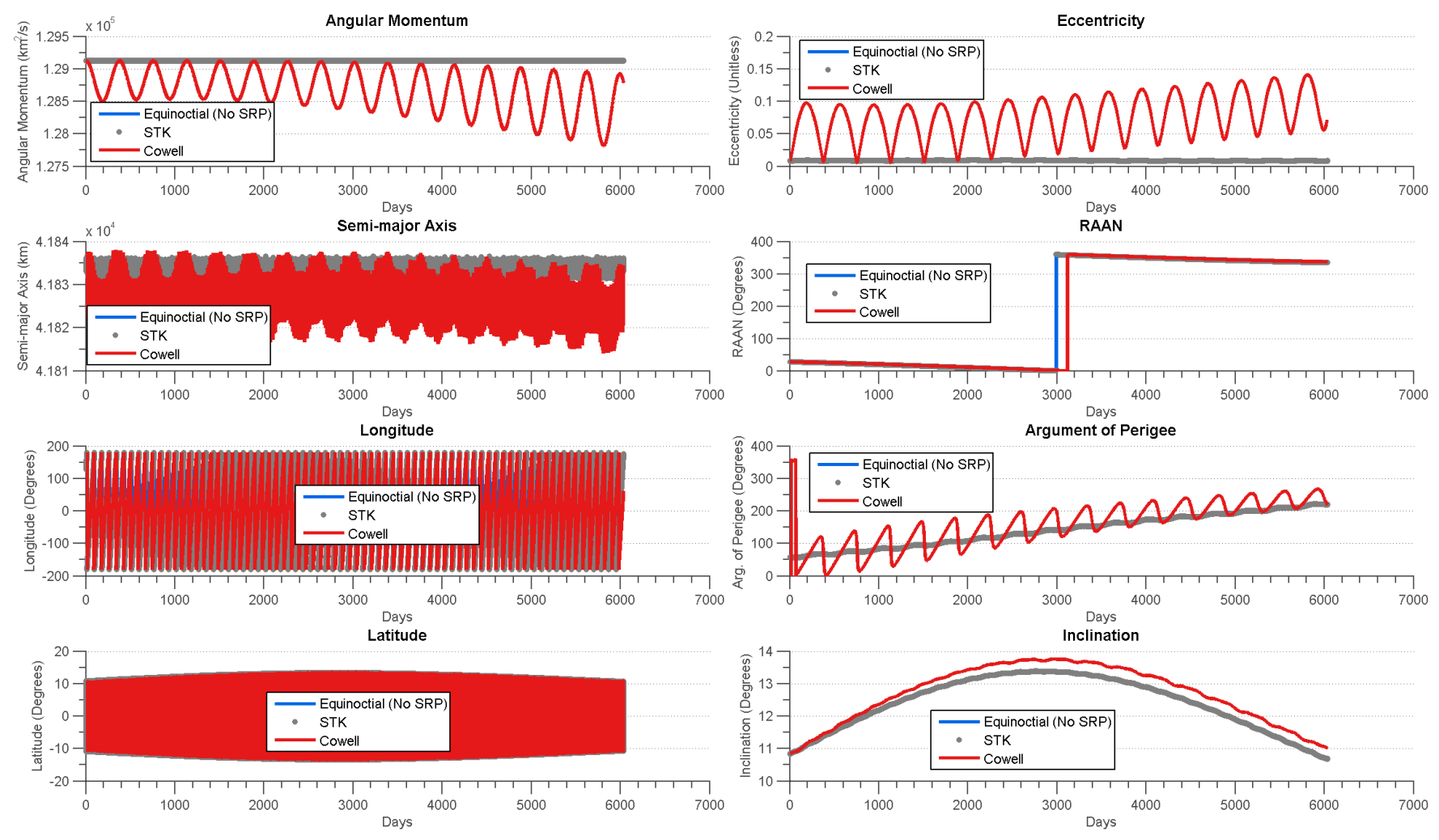

Figure A.1: Long Term No-SRP Plots for Satellite 03432 

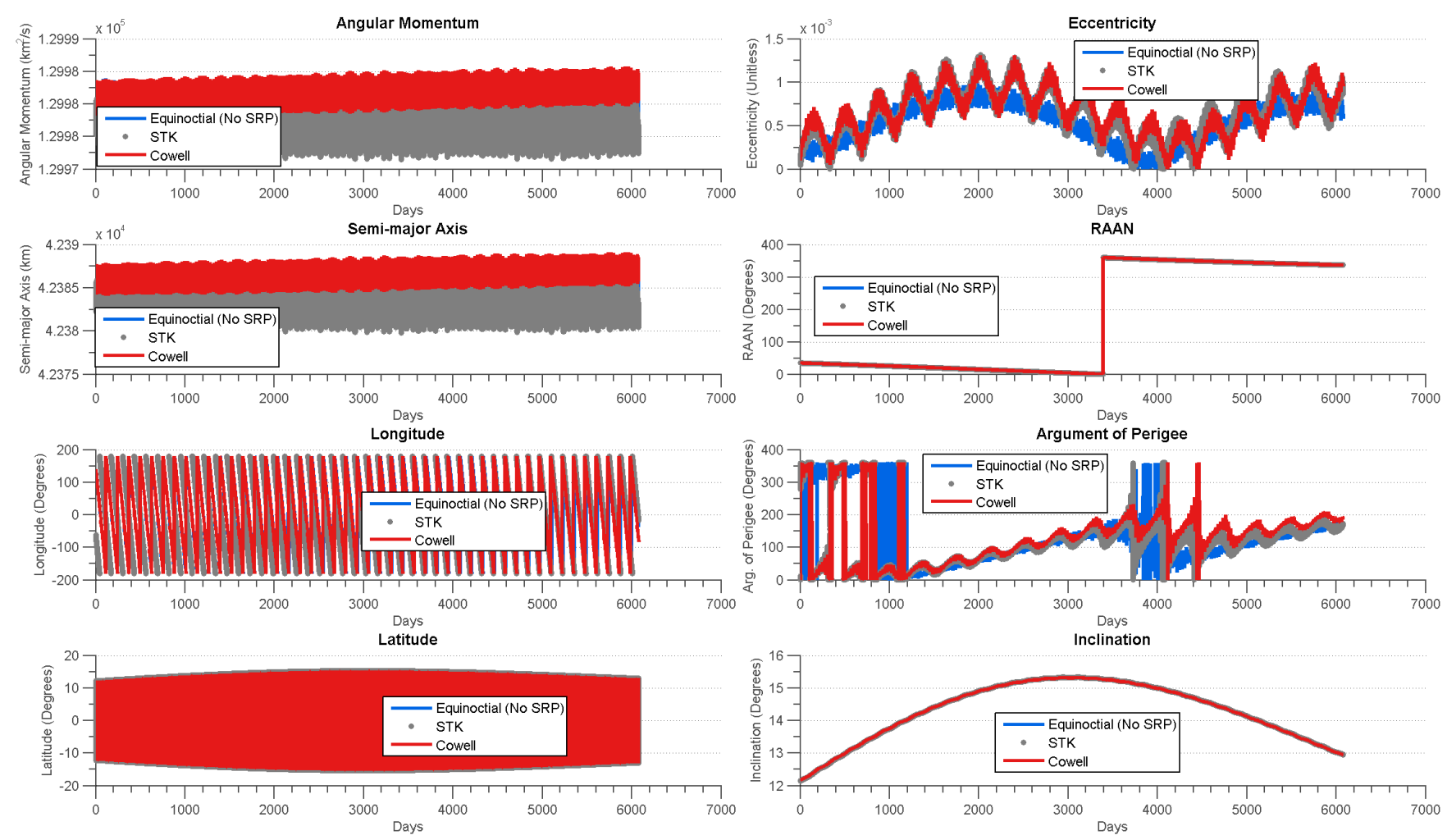

Figure A.2: Long Term No-SRP Plots for Satellite 04068 

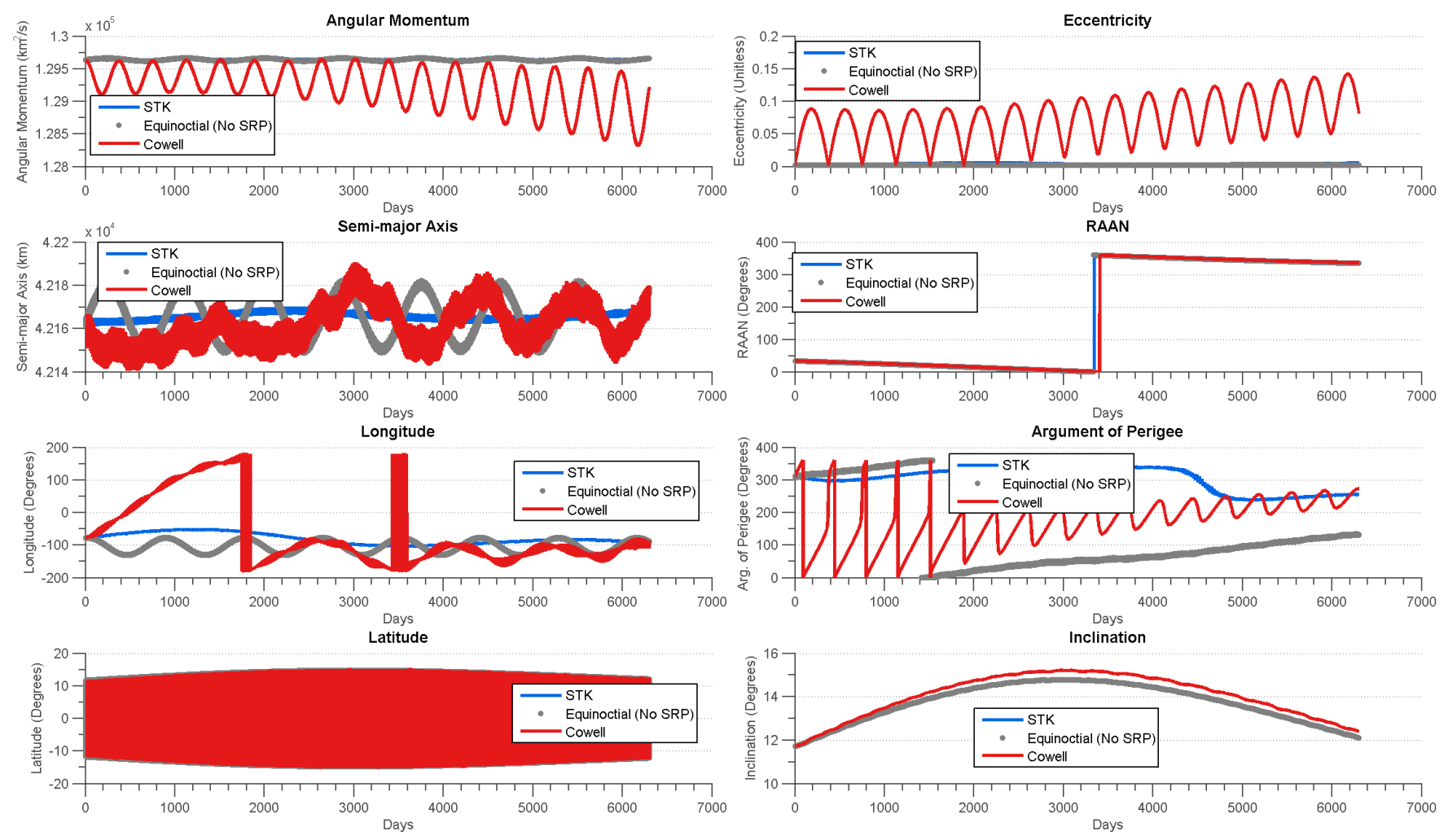

Figure A.3: Long Term No-SRP Plots for Satellite 04250 

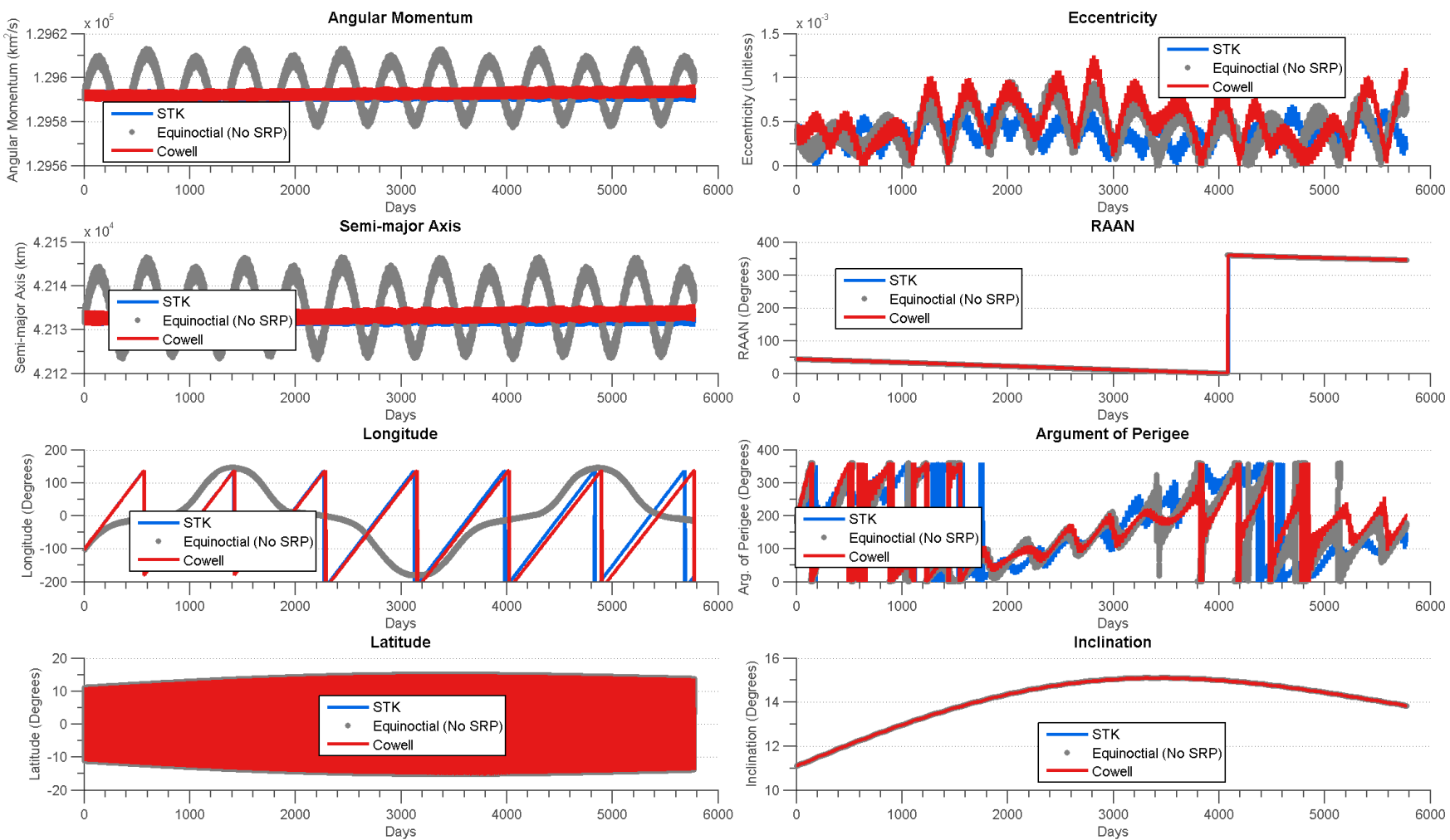

Figure A.4: Long Term No-SRP Plots for Satellite 05588
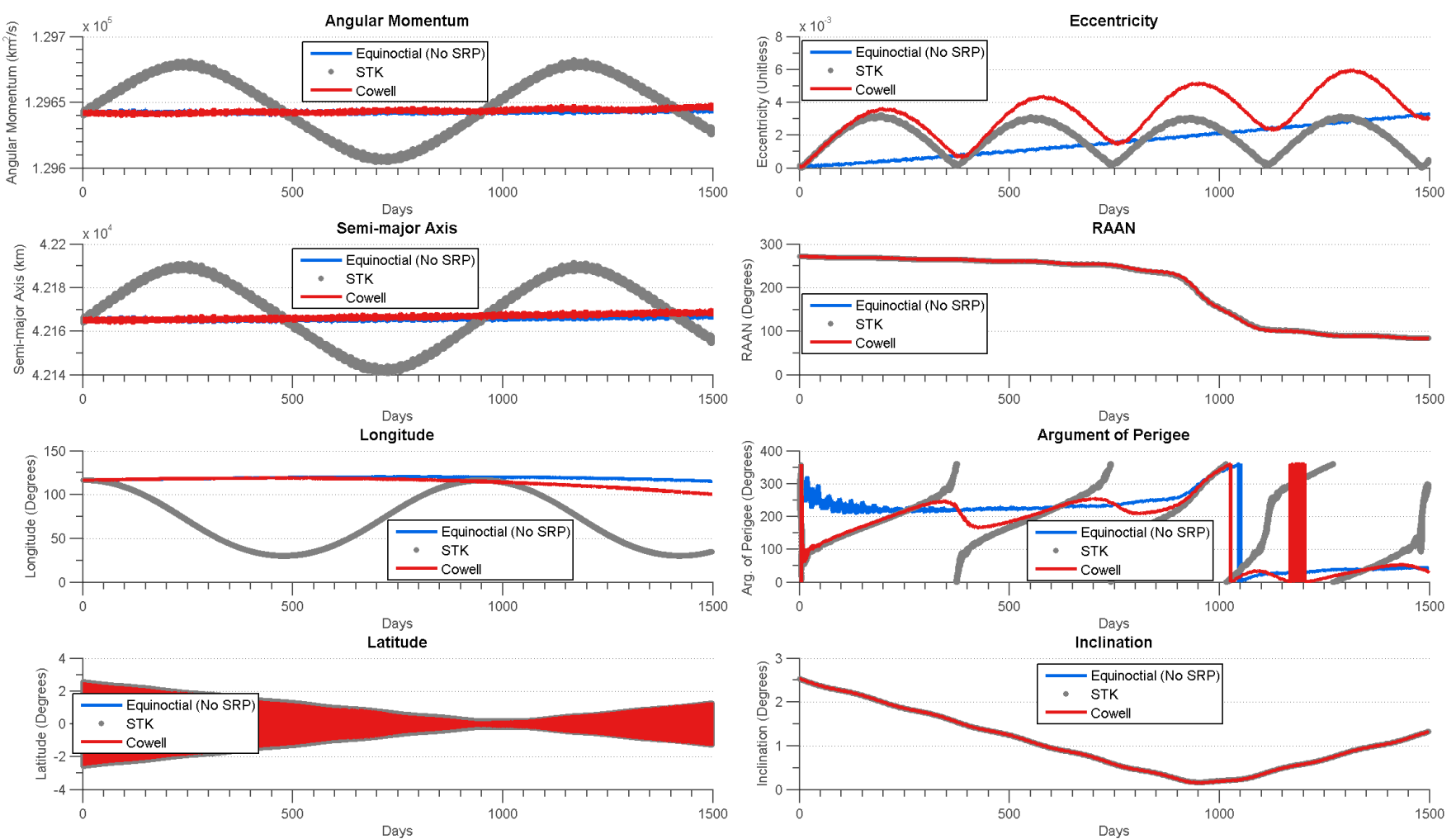

Figure A.5: Long Term No-SRP Plots for Satellite 29640 


\section{B. Comparing Cowell Propagators}
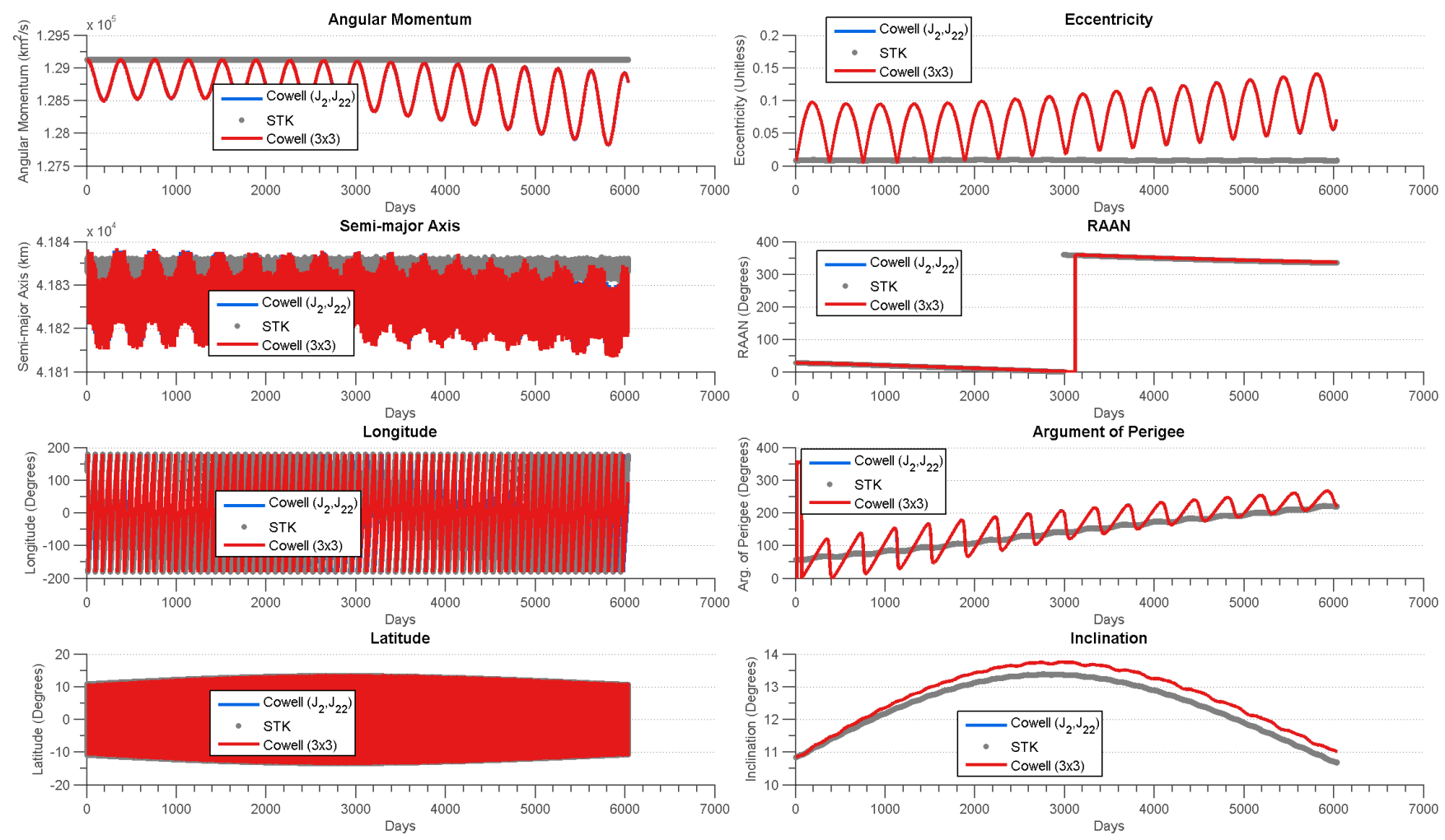

Figure B.1: Cowell Comparison Plots for Satellite 03432 

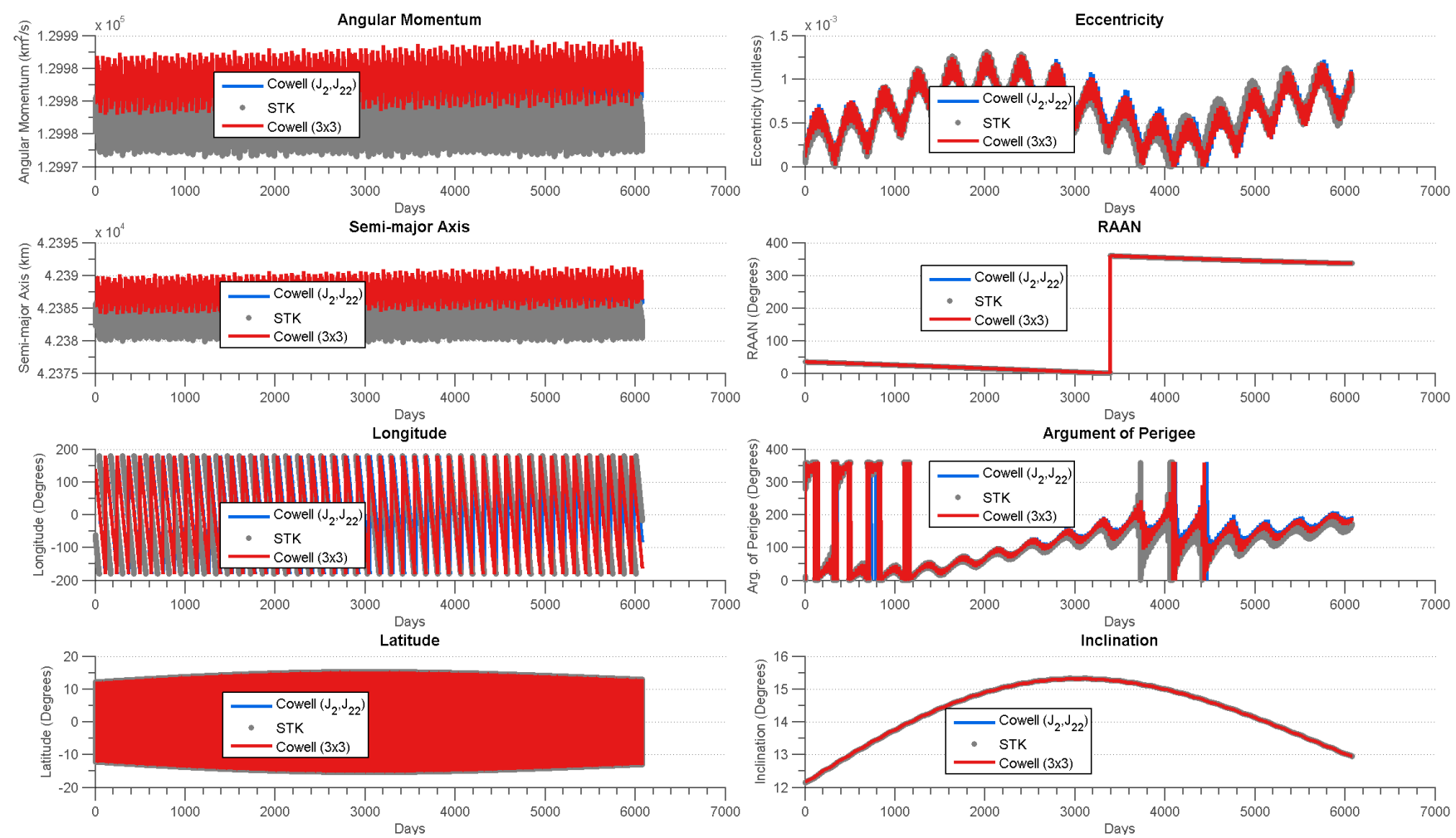

Figure B.2: Cowell Comparison Plot for Satellite 04068
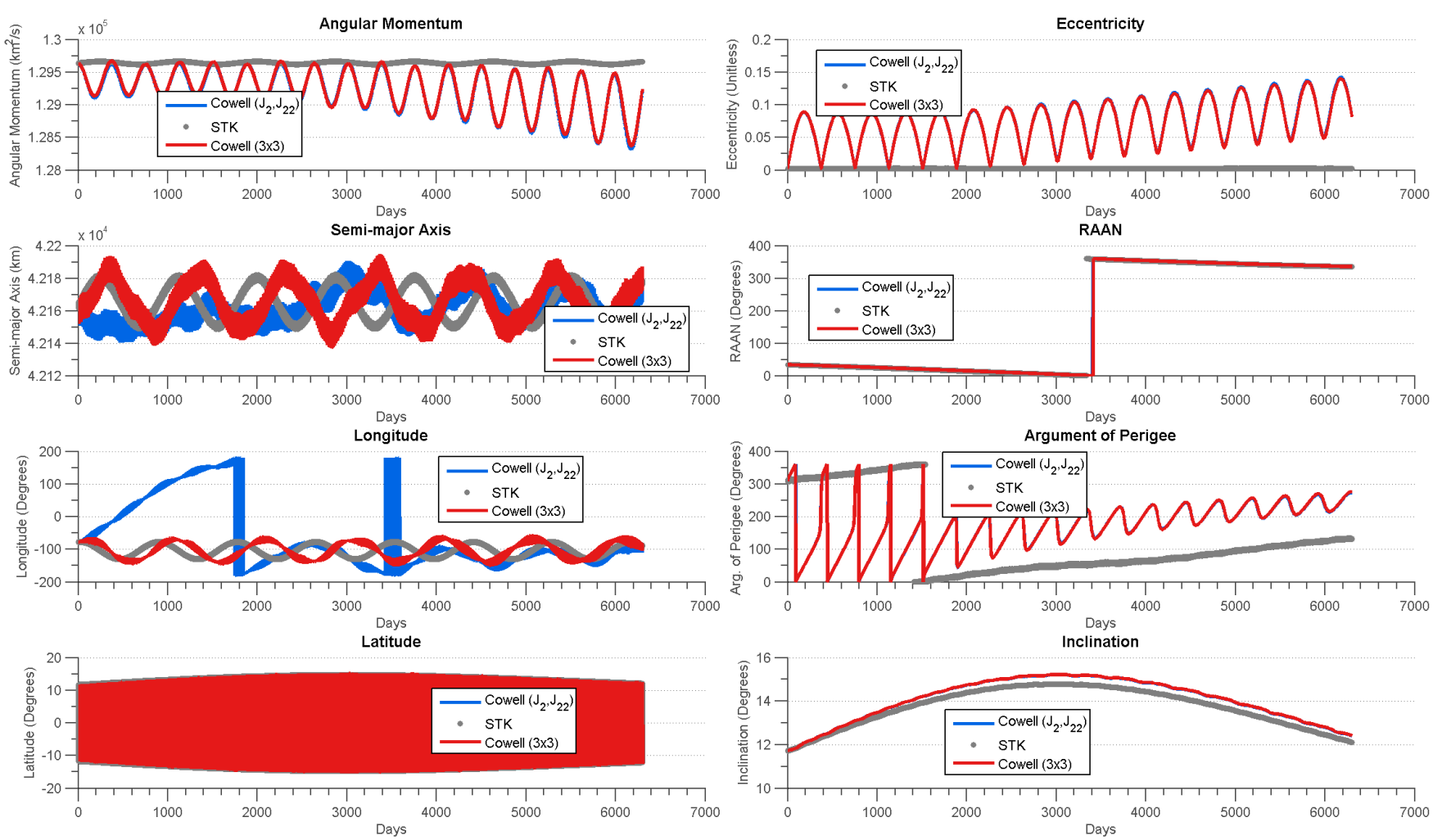

Figure B.3: Cowell Comparison Plot for Satellite 04250 

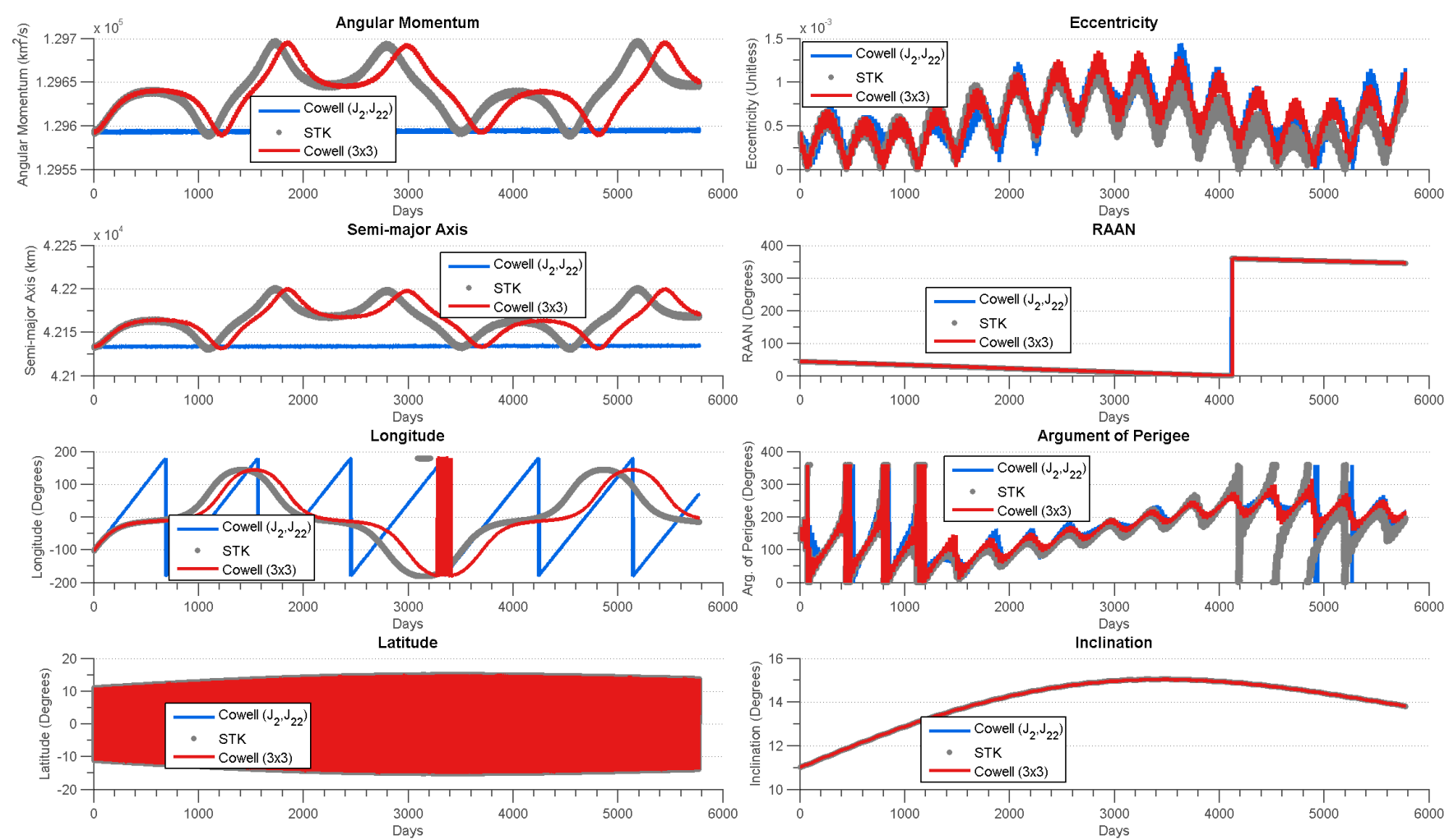

Figure B.4: Cowell Comparison Plot for Satellite 05588
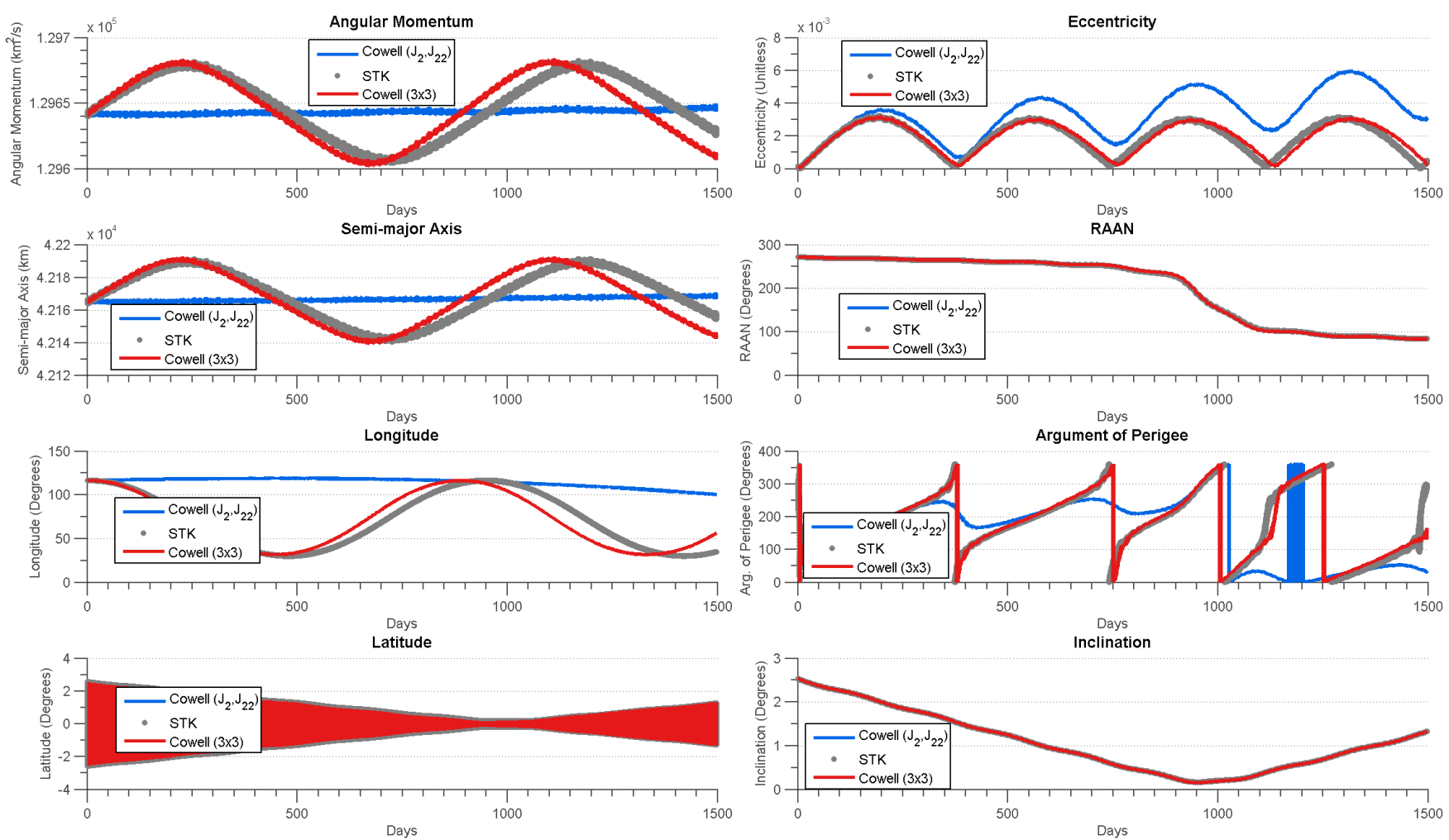

Figure B.5: Cowell Comparison Plot for Satellite 29640 